2012

\title{
A Physiological Signal Processing System for Optimal Engagement and Attention Detection.
}

Ashwin Belle

Virginia Commonwealth University

Follow this and additional works at: https://scholarscompass.vcu.edu/etd

Part of the Computer Sciences Commons

(C) The Author

\section{Downloaded from}

https://scholarscompass.vcu.edu/etd/394

This Dissertation is brought to you for free and open access by the Graduate School at VCU Scholars Compass. It has been accepted for inclusion in Theses and Dissertations by an authorized administrator of VCU Scholars Compass. For more information, please contact libcompass@vcu.edu. 
(C) Ashwin Belle 2012

All Rights Reserved 


\section{A PHYSIOLOGICAL SIGNAL PROCESSING SYSTEM FOR OPTIMAL ENGAGEMENT AND ATTENTION DETECTION}

A dissertation submitted in partial fulfillment of the requirements for the degree of $\mathrm{PhD}$ in Computer Science at Virginia Commonwealth University.

by

ASHWIN BELLE

Master of Science in Computer Science, Virginia Commonwealth University, 2008

Bachelors in Computer Science and Engineering, DSCE, India, 2007.

Director: KAYVAN NAJARIAN

ASSOCIATE PROFESSOR, DEPARTMENT OF COMPUTER SCIENCE SCHOOL OF ENGINEERING.

Virginia Commonwealth University

Richmond, Virginia

July 2012 


\section{Acknowledgement}

I would like to take this opportunity to first acknowledge my parents, Ashok Belle and Anu Ashok, for their unwavering faith in me and their immortal love. Their support and guidance has given me an extraordinary platform to pursue and achieve any and all of my ambitious dreams and targets.

Next, my deepest and most sincere gratitude goes out to my advisor Dr Kayvan Najarian. I have been extremely fortunate to have him as my advisor since he has been a pillar of strength and wisdom for my $\mathrm{PhD}$ education, research and much more. I would also like to extend my sincere thanks to my committee members, who have so kindly agreed to be on my committee despite knowing that they will be required to patiently read my entire thesis manuscript and sit through my presentation that follows. On a more serious note I do appreciate their support and help towards accomplishing my PhD education.

For this research collecting data was essential, and so I like to wholeheartedly thank all the volunteer subjects who patiently sat through hours and hours of data collection, all while being strapped to various bio-medical signal acquisition machines. I especially thank those who had to sit through these experiments more than once due to machine malfunctions. Finally I would like to thank Michael Pfaffenberger, a fellow PhD student, who has been great help with certain aspects of this project. 


\section{Contents}

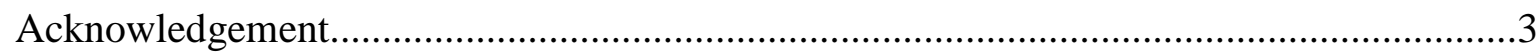

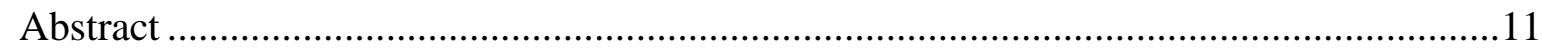

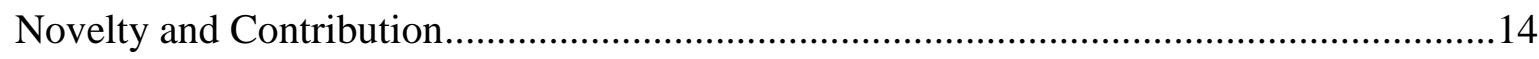

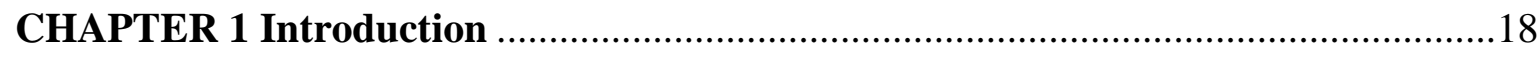

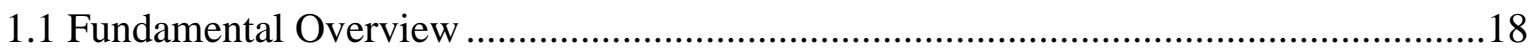

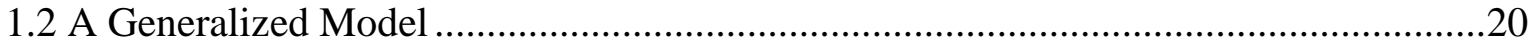

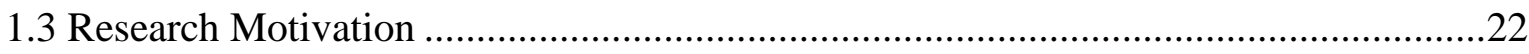

CHAPTER 2 Background and Application Prospects ...........................................26

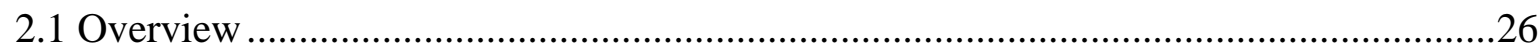

2.2 Application specific need for attention aware systems .........................................28

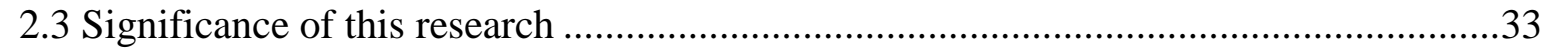

CHAPTER 3Experimental Setup and Description of Dataset.................................35

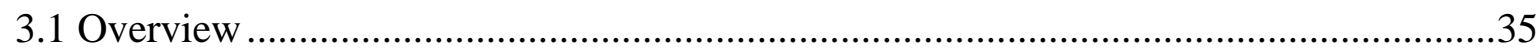

3.2 Description of the conducted experiment ..........................................................36

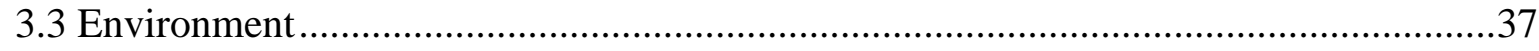

3.4 Physiological Signal Acquisition .................................................................38

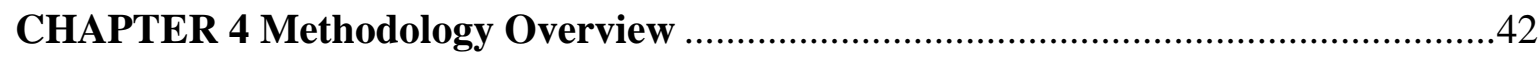

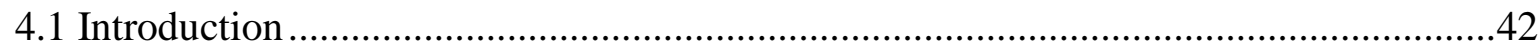


5.1 Overview 51

5.2 Analysis Methods .54

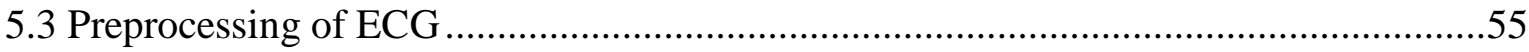

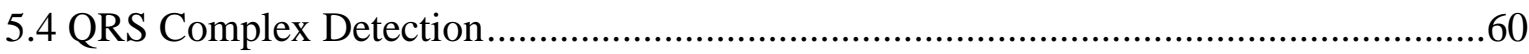

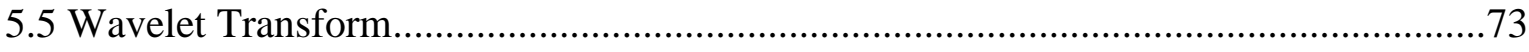

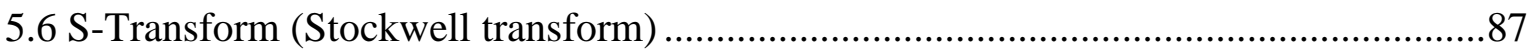

5.7. Dual Tree Complex Wavelet Transform -An Introduction. .....................................96

CHAPTER 6 Analysis of Electroencephalogram ...............................................106

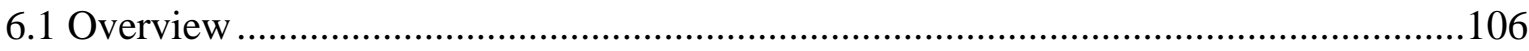

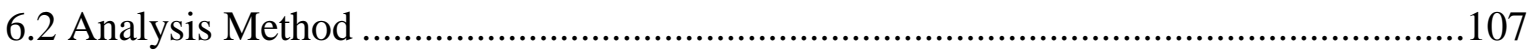

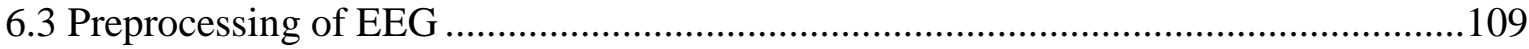

6.4 Applying Discrete Wavelet Transform on EEG ...............................................114

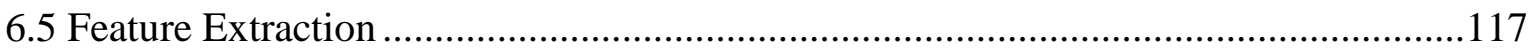

CHAPTER 7 Analysis of other physiological signals: Galvanic Skin Response and Heat Flux 119

7.1 Analysis of Galvanic Skin Response 119 
7.2 Analysis of Heat Flux

122

CHAPTER 8 Machine Learning and Classification Model ...................................125

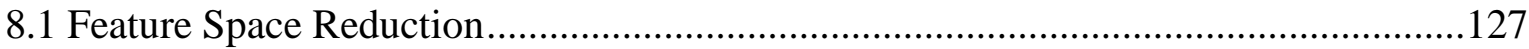

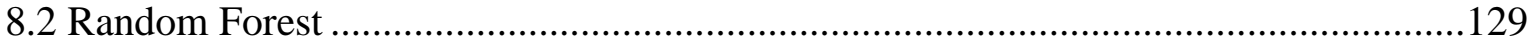

8.3 Parameter Selection for Random Forest ..........................................................132

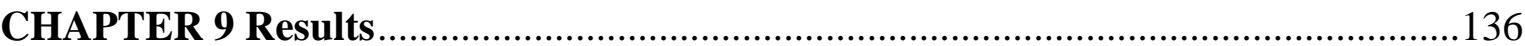

9.1 Results from Individual Analysis Methods........................................................138

9.2 Comparison of ECG and EEG analysis based on individual subjects ......................148

9.3 Results developed towards practical application ..............................................157

9.4 Experimental setup validation using EEG features................................................164

CHAPTER 10 Conclusion and Future Work.................................................168

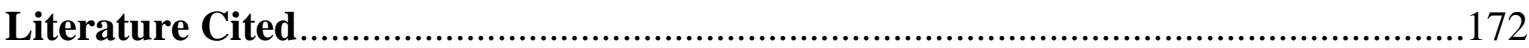

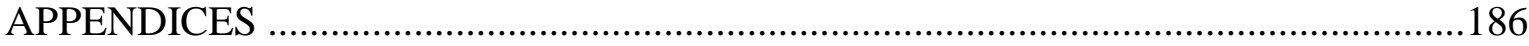

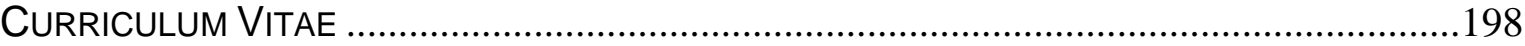


Table of Tables

Table 1. Confusion matrix Template ......................................................................136

Table 2: DWT Classification Accuracies by subject ...............................................139

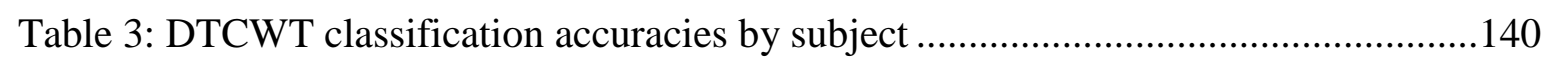

Table 4: S Transform classification accuracies by subject ........................................141

Table 5: HRV values classification accuracies by subject..........................................143

Table 6:Combined Featureset of ecg classification accuracies by subject .......................144

Table 7: EEG Analysis classification accuracies by subject........................................146

Table 8: ECG and EEG analysis classification accuracies comparison by subject ..........149

Table 9: Table of overall performance perspective accross 13 subject cases with both ECG

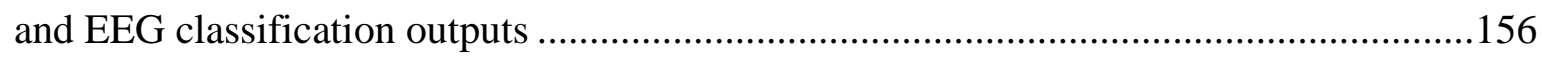

Table 10: Model accuracies obtained by step-size Ranked features.............................160

Table 11: Classification accuracies for each subject.................................................161

Table 12: Classification output comparison table before and after feature space reduction 


\section{Table of Figures}

Figure 1. A generalized model .............................................................................21

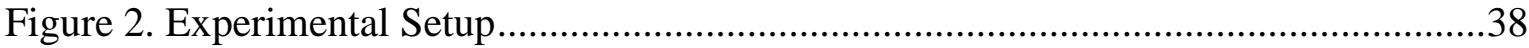

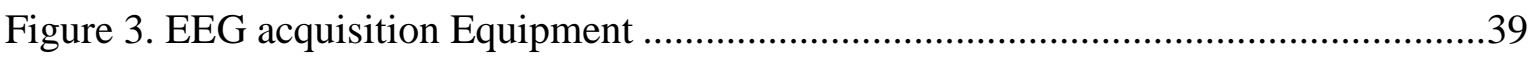

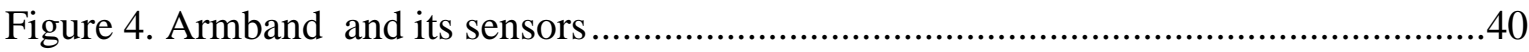

Figure 5. Raw Based signal analysis Approach ......................................................43

Figure 6. Feature Based signal analysis Approach .................................................44

Figure 7. Decomposition Based signal analysis Approach ..........................................45

Figure 8. Model based signal analysis Approach ....................................................46

Figure 9. Research Methodology Overview …..............................................................48

Figure 10. (A) ECG Signal, (B) Cardiac Complex .......................................................52

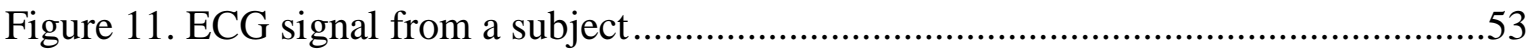

Figure 12. Analysis Implementation procedure .....................................................54

Figure 13. ECG signal preprocessing...............................................................56

Figure 14. (a) Before noise removal, (B) After noise removal ......................................57

Figure 15. ECG baseline drift removal, before and after ...........................................58

Figure 16. QRS Complex detection steps .........................................................60

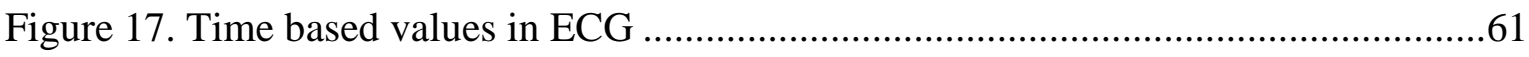

Figure 18: Central Nervous System layout ...........................................................69

Figure 19. Discrete Wavelet Transform Application steps ..........................................77

Figure 20. (a) is the sample ECG wave, (b) is the db4 Wavelet function, (c) Decomposition Low-Pass filter for db4, and (d) Decomposition High-pass filter for db4 ..........................80 
Figure 21. Decomposition process of Wavelet transform .81

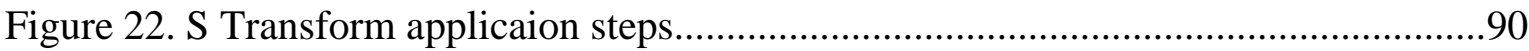

Figure 23. Contour based visualization of an ST window ..........................................92

Figure 24. Dual tree wavelet Analysis illustration...................................................98

Figure 25. Dual tree wavelet Synthesis illustration. .................................................99

Figure 26. EEG processing methodology ...........................................................108

Figure 27. (a)Raw EEG signal (b) EEG frequency response......................................110

Figure 28. Delta Wave illustration ..........................................................................110

Figure 29. Theta Wave Illustration ....................................................................111

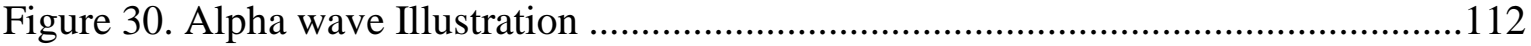

Figure 31. Beta Wave Illustration ......................................................................112

Figure 32. EEG preprocessing step ................................................................113

Figure 33. (a)‘coif3’ wavelet (b) ‘db4’ wavelet and (c) ‘boir3.9’ wavelet........................116

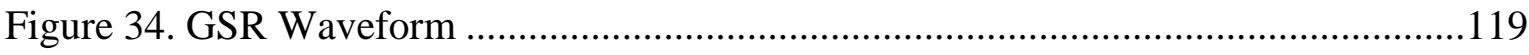

Figure 35: Dynamic Computation of number of trees ................................................133

Figure 36: Computation of depth of trees dynamically ............................................135

Figure 37: Case 1 Instance based comparison of classification output between (a) ECG and

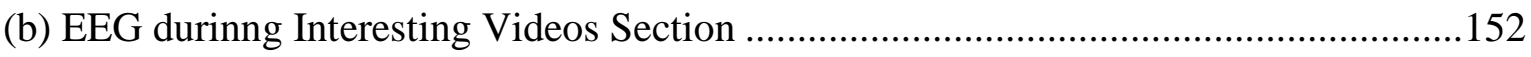

Figure 38: Case 1 instance based comparison of classification output between (a) ecg and (b) eeg durinng boring videos section 153

Figure 39: Case 2 instance based comparison of classification output between (a) ecg and (b) eeg durinng interesting videos section 154 
Figure 40: Case 2 instance based comparison of classification output between (a) ecg and

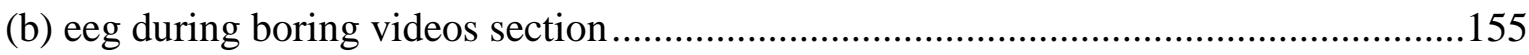
Figure 41: Feature Ranking, where x-axis is 394 features numbers and yaxis is number of

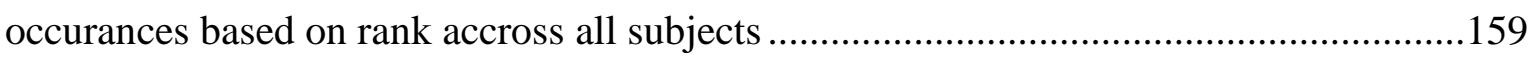
Figure 42: Receiver operating characteristic of the Classification Model after feature

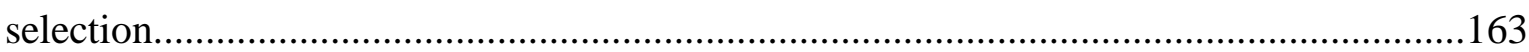

Figure 43: Hybrid feature selection routine ...........................................................165 Figure 44: Feature Ranking Based on Each Band Component......................................167 


\begin{abstract}
A PHYSIOLOGICAL SIGNAL PROCESSING SYSTEM FOR OPTIMAL ENGAGEMENT AND ATTENTION DETECTION

By Ashwin Belle
\end{abstract}

A dissertation submitted in partial fulfillment of the requirements for the degree of $\mathrm{PhD}$ in Computer Science at Virginia Commonwealth University.

Virginia Commonwealth University, 2012

Major Director: Kayvan Najarian

Associate Professor, Department of Computer Science, School of Engineering

In today's high paced, hi-tech and high stress environment, with extended work hours, long to-do lists and neglected personal health, sleep deprivation has become common in modern culture. Coupled with these factors is the inherent repetitious and tedious nature of certain occupations and daily routines, which all add up to an undesirable fluctuation in individuals' cognitive attention and capacity. Given certain critical professions, a momentary or prolonged lapse in attention level can be catastrophic and sometimes deadly. 
This research proposes to develop a real-time monitoring system which uses fundamental physiological signals such as the Electrocardiograph (ECG), to analyze and predict the presence or lack of cognitive attention in individuals during task execution. The primary focus of this study is to identify the correlation between fluctuating level of attention and its implications on the physiological parameters of the body. The system is designed using only those physiological signals that can be collected easily with small, wearable, portable and non-invasive monitors and thereby being able to predict well in advance, an individual's potential loss of attention and ingression of sleepiness. Several advanced signal processing techniques have been implemented and investigated to derive multiple clandestine and informative features. These features are then applied to various machine learning algorithms to produce classification models that are capable of differentiating between the cases of a person being attentive and the person not being attentive. Furthermore, Electroencephalograph (EEG) signals are also analyzed and classified for use as a benchmark for comparison with ECG analysis.

For the study, ECG signals and EEG signals of volunteer subjects are acquired in a controlled experiment. The experiment is designed to inculcate and sustain cognitive attention for a period of time following which an attempt is made to reduce cognitive attention of volunteer subjects. The data acquired during the experiment is decomposed and analyzed for feature extraction and classification. The presented results show that to a fairly reasonable accuracy it is possible to detect the presence or lack of attention in individuals with just their ECG signal, especially in comparison with analysis done on EEG signals. The continual work of this research includes other physiological signals such 
as Galvanic Skin Response, Heat Flux, Skin Temperature and video based facial feature analysis. 


\section{Novelty and Contribution}

Cognitive psychology is based on a person's ability to acquire process and store information; which applies fundamentally to most of the conscious tasks humans undertake. The crux of this hypothesis lies in the capacity of attention and engagement levels that a person is able to inculcate and sustain during a cognitive activity. The more attentive and engaged a person is while carrying out a given task, the better the yields are in the performance of that individual. The strength and sustainability of attention has so far been a non-determinant variable which shows high levels of dependency and correlation with several environmental and inherent factors that is specific to each individual.

Several critical and non-critical occupations demand a certain level of consistent performance which happens to be highly correlated with the individual's attention levels. Momentary lapse of attention while performing certain critical tasks such as surgery, piloting a plane, driving, operating heavy machinery, etc. could lead to some devastating outcomes. Human errors due to drowsiness or lack of focus are a common contingency at workplaces which can lead to very expensive consequences. In many cases such outcomes are not entirely imperative and can be avoided given the means to successfully monitor and predict the onset of drowsiness much in advance, thereby allowing sufficient time to practice certain precautionary measures.

This proposed study is involved in developing one such system that can be used to perform real time monitoring and classification of various physiological signals thereby 
providing the ability to predict the fluctuations in cognitive attention levels and the onset of drowsiness in advance. There is some research currently in this area which uses physiological and non-physiological data to recognize a person's cognitive status. However, most of these researches either use a single modal for estimation or are not specifically targeting attention in individuals. Most of these studies are established based on certain specific contingencies which are not designed for all purpose application. As compared to existing systems and research in this realm, this proposed system boasts a few novelties and contribution that sets it apart from the rest. These novelties are as follows;

Multi-modal perspective: In contrast to existing studies, this proposed system is designed to encompass several physiological modals for investigating the existence or absence of cognitive attention. The study investigates physiological signals such as Electrocardiogram (ECG), Heat Flux (HF), and Galvanic Skin Response (GSR) to recognized specific psychological parameters especially those of attention and engagement. Advanced signal processing techniques are exploited to examine the influence that diluting attention has on physiological signals. Electroencephalogram (EEG) signals which are proven as effective means for studying cognitive activities are utilized mainly to establish a benchmark for the analysis outcomes of the other signals used in this study.

Extensive ECG analysis: As mentioned earlier, ECG and other mentioned physiological measures used in this study have not commonly used for the purpose of recognizing attention. Especially the ECG although used for many other bio-medical applications, it has seen very little application in this area previously. With this bearing, in this study the 
ECG signal is being studied in extensive detail for recognizing attention specific implication on its characteristics. Several ingrained and cutting-edge signal processing approaches have been utilized in a creative fashion to compute substantial and valuable features from the ECG signal in attempt to identify cognitive attention.

Portable Architecture: The proposed system has been distinctly designed and developed for practical application in real world settings. With comparable systems that are designed using EEG signal, it is immediately evident that portability of these systems are restricted, thereby limiting and confining its applications to fixed and controlled settings. This proposed system on the other hand relies on data that can be solely collected using a wearable, wireless, non-invasive and completely portable armband which when in use has a small concealable footprint on the body. The system's portable architecture allows it to be applicable in variety of real world settings and applications. Furthermore, for continuous monitoring of attention and prolonged use, the small footprint of the armband on the body makes it extremely easy to use for extended periods of time. While being completely non-invasive yet concealable.

Real-time framework: Another important aspect of the design of this proposed system is in its real-time application framework. The system is designed to provide real-time analysis and feedback based on its prediction and classification. With a sufficient large training data-set, a model can be trained and developed which can be applicable towards general usage. The trained classification model is also going to be developed to be less 
sensitive to inter-person variability which is common with such multi-modal physiological experiments.

Innovative feature extraction: With the various signal transposition and decomposition methods being used, several domain specific features are being extracted for exploiting such processing techniques. Common statistical features as well as domain based heuristic features have been designed for this study.

Machine Learning Advances: To enhance the quality of feature classification and the accuracy of prediction, feature quality evaluation methods and classification techniques are implemented and assayed to ensure the development of the best classification model based on the nature of application. 


\section{CHAPTER 1 Introduction}

\subsection{Fundamental Overview}

This research investigates, with an engineering perspective, a fundamental phenomenon called 'cognitive attention', which is an inherent aspect of human psychology. In our everyday life, amidst all the information that is encumbered on our sensory mechanisms, only a fraction of this is processed by the brain and even fewer of them receive a response from it. So the obvious question that arises is how does the human brain select what it decides to pay 'attention' to and by how much? And when does the brain start declining the level of attention it is paying?

Attention as a quintessential subsistence has been recognized and analyzed for centuries now. Early philosophers, like Descarates [1], Berkeley [2], Locke [3] wrote extensive theories about the existence, role and affects of cognitive attention. Even luminaries such as Confucius and Leonardo da Vinci are said to have discussed and described the conscious existence of attention. In the recent past however, the idea of cognitive attention has gained much more conglomerated interest and has been examined in various angles by different branches of study such as psychology, psychiatry, neurology, behavioral science, philosophy and not to forget engineering. 
In past few centuries there have been significant research on the concept of attention. Although as humans our perception and concept of attention seems fundamental and easily definable (primarily basing it through own experiences), however for the scientific community there is no single way of defining the word attention. We all experience that, to some extent our awareness and control of our current attention depends on what we choose to attend to. For example while reading this document you have chosen to focus on the content of this document as opposed to several other entities around you such as the faint noise of the vents in the background, the sounds from outside etc. However, after reading this document for long enough, your attention is bound to fluctuate from its content to other distractions that surround you. Prolonged attempts to read this document entirely in one sitting might possibly make you drowsy and put you to sleep. The ability to sustain ones attention, and the frequency of fluctuation or fading of one's attention could be due to a combination of multiple reasons. Factors that affect attention are lack of sleep, medications, emotional state, distractions of the environment, fatigue, so on and so forth. Recognizing that the affect state of attention is not definable given its complex nature of interactions between the physical, physiological and environmental entities, this research is not concerned about the sources and causes of attention related issues. Instead this study attempts to understand the 'everyday experience of attention', that is, the existence and/or the lack of, attention during our everyday activities.

In today's high paced, hi-tech and high stress environment, a common sufferer is our cognitive processing and capacity. Extended work hours, long to-do lists and neglected health could cause sleep deprivation, a commonplace occurrence in modern culture. 
Coupled with these factors is the repetitious nature of the activities themselves that we perform on a daily basis. Such as long hours of driving or commuting, fragmented or routine jobs, lack of cognitive demand at work place due to automation and machining, etc. The result of such daily tautological and tedious routines in our lives results in something that is widely recognized as boredom and monotony which are readily agreed as undesirable and unpleasant states. During the recent years investigators have begun to refer to monotony and boredom not just as undesirable aspects of modern life, but also as a stressor that may be as potentially harmful to the individual [4][5][6][7][8][9].

The study is purely based on an engineering perspective and is focused on deriving the correlation between cognitive attention and physiological signals of the human body. The concept of this research is to use advanced signal processing techniques to develop a mathematical expression of cognitive attention and its effects on certain key human physiology, thereby being able to analyze, enumerate, categorize and predict the presence or absence of attention during cognitive activities.

\subsection{A Generalized Model}

The following model is developed to explain the fundamental hypothesis on which this research stems from. This model was developed based on literature review of various fields. It is to be noted that this model does not exhaustively represent all the elements of 
attention; it is instead a simple abstraction of some common real world factors being considered in this study.

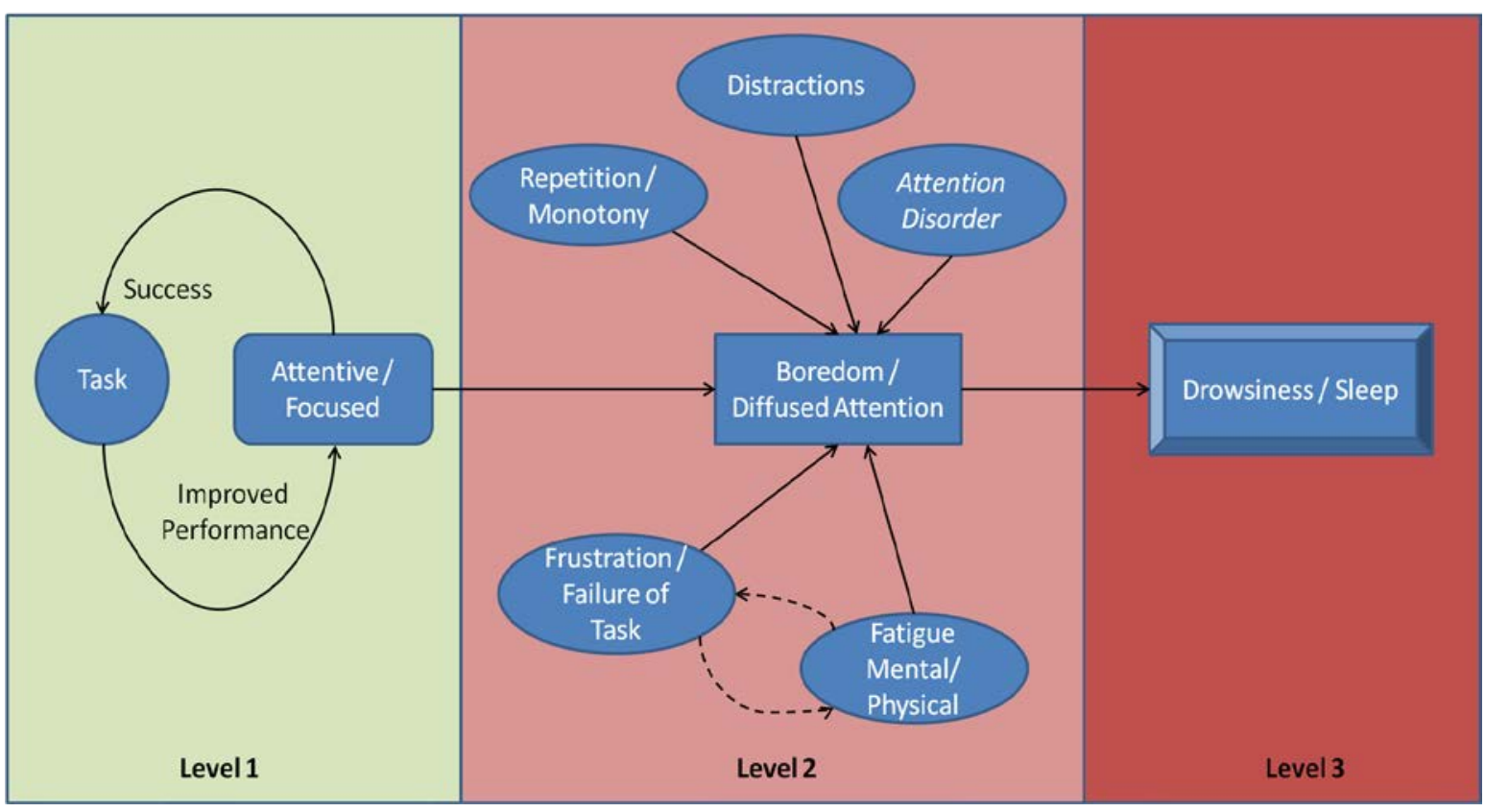

FIGURE 1. A GENERALIZED MODEL

As the model depicts, level 1 is where the individual undertakes a task and is focused on the task. The words 'focus' and 'attention' could arguably mean different in different settings, however with respect to this study it is to be used interchangeably. In level 1, it can be seen that the presence of attention provides improved performance of the task and thereby results in success of the task, intuitively it can also be seen that due to the success in task and performance gain of an individual, the level of attention seems to benefit inherently as well. 
Level 2 is when the individual moves from a state of being focused/attentive to a state of boredom/diffused attention. This change in state of cognitive attention could be due to a number of reasons; however a generalized few factors have been shown in the depiction of the model. Monotonous or repetitive nature of the task, distractions or external stimuli, inherent attention disorder in the individual are some of the common causes of fluctuations between attentive and non-attentive states. Fatigue and frustration are two special factors that by themselves are sometimes primary causes for distorting attention but also could be results of other causes. There exists multiple obvious inter-relationships within these factors; an example of this would be that, a fatigued person, doing a repetitive task can get easily distracted and fail to perform adequately which could lead to frustration which in turn could cause further mental fatigue which finally leads to level 3 that is sleep drowsiness or sleep.

\subsection{Research Motivation}

With the viewpoint of the task at hand, the final stage that is drowsiness or sleep can be a very abominable state. A body of literature exists on the mechanism of human sleep and sleepiness. Homeostasis relates to the neurobiological need to sleep. The sleep-wake cycle is governed by both homeostatic and circadian factors, where the circadian pacemaker is an internal body clock that completes a cycle approximately every 24 hours. The longer the humans pressure themselves against sleep, the more difficult it becomes to resist it [10]. The sleep-wake cycle is intrinsic and inevitable, not a pattern to which people voluntarily 
adhere or can decide to ignore [11]. Training, occupation, education, motivation, skill level, and intelligence exert no influence on reducing the need for sleep [12]. It is known that sleepiness impairs human performance and relevant impairments are identified in this study by Dinges and Kribbs [10]. The identified impairments are as follows;

- Slower reaction time

- Reduced vigilance

- Deficits in information processing

Often people use physical activity and dietary stimulants to cope with sleep loss, to mask their need to sleep. However, when they sit still, perform repetitive tasks (such as driving long distances), get bored, or let down their coping defenses, sleep comes quickly $[13,14]$.

Depending on professions, a momentary or prolonged lapse in attention level can be catastrophic and sometimes deadly. For example, Federal Aviation Administration (FAA) has had to suspend many of their air traffic controllers and have been forced to make amendments to their work cycle policies since many of their air traffic controllers were either caught sleeping or making calamitous mistakes on their jobs due to being over worked and fatigued. An article in the Washington post in 2010 by A. Halsey highlighted that the errors recorded by air traffic controllers had recently increased by 51 percent nationwide. In April 2011, Reno-Tahoe International Airport, Nevada, when an airambulance plane was franticly trying to land with a critically ill patient on board, the pilot received no assistance from the traffic controller who had fallen asleep. After nearly 20 minutes of waiting for assistance the pilot was forced to land without guidance and the 
sleeping air traffic controller was subsequently suspended. This was the 5th such incident recorded in the country between January and April of 2011 alone.

Similarly there are several traffic and road accidents being recorded around the country regularly due to drowsy or sleeping drivers. May 2011 in Virginia, a bus crash killed several and wounded many more of its 52 passengers when the driver of the bus fell asleep at the wheel. A similar incident in March of 2011 near New York City killed 15 passengers when the driver was asleep as the bus drifted off its lanes and violently crashed on I-95. American Automobile Association (AAA) Foundation for Traffic Safety poll in 2010 [15] found that $41 \%$ admitted to have nodded off or completely fallen asleep while they were driving in the past year. Although it might seem that it's more common to doze off during long car rides, it turns out that 59\% said they'd been driving under an hour before they had fallen asleep and only $20 \%$ had been driving for more than three hours.

These are just a few recent examples of the horrible effects of lack of attention or sleepiness during certain task performance. Other critical profession such as doctors, security personnel's, police officers, soldiers, pilots etc, have also reported several incidents each year that is in one way or another attributed to loss of attention, fatigue and/or sleepiness.

Motivated to solve this common problem, this research is an attempt to use fundamental physiological signals which could be collected easily with tiny wearable and portable monitors and thereby being able to predict well in advance, an individual's potential loss of attention and ingression of sleepiness during a task execution. It is 
expected that a successful application of this research will help recognize patterns in individual task performance, improve performance and perhaps in turn save lives.

The research as stated before is purely an engineering and mathematical perspective of recognizing the presence or the lack of attention. With respect to the depicted general model in the above figure, this research is trying to recognize the physiological changes of an individual when the individual shifts from Level 1 through Level 3 during task performance. 


\section{CHAPTER 2 Background and Application Prospects}

\subsection{Overview}

Studying alertness and drowsiness is not a new domain in scientific research. Numerous research areas are actively studying the concepts of attention or alertness, distraction, drowsiness etc. Research of attention and drowsiness is multifaceted and most of the research is highly disciplinary based or application specific. Literature review shows that there seems to be as many different concepts of what affects and controls cognitive attention in humans as there are researchers in this field.

Some studies consider mental fatigue as the primary factor of reduced attention or drowsiness, and hence try to detect the direct effects of fatigue based on mood, behavior and information processing $[16,17]$. Research correlating attention to depression and chronic fatigue syndrome which is co-morbid to a wide range of psychological and somatic

disorders have also been done in the past $[18,19]$. Evidence relating to diminishing cognitive or voluntary control over attention accompanied by fatigue has been established in research by Lorist et al., Sanders, Van der Linden et al.[20-22]. Some research suggests a direct link between sensorimotor gating and cognitive control of attention and how one affects the other under fatigue $[23,24]$. 
Several studies describe roles of stress on alertness and attention of individuals. Publications such as [25-30] try to determine the effects of stress on human performance. There are multiple researches focusing on work environments and the impact different workloads have on attention levels [31, 32, 32-34].

Similarly several non-sensory based mechanisms have also been developed to identify and quantify attention level in individuals. Non- sensory inputs such as user's daily routine, schedules, activities, with self reports from users describing patterns in activities and attention levels during them, have also been designed to develop various models of attention, as in daily life [35-38]. Human attention has been studied keenly by multi disciplines. From literature review it can be seen that research and research results are scattered and interdisciplinary work in this area is limited. A new wave of interest can now be seen in the development of 'attention aware systems', i.e. systems which are perceptive of the user's attention and possibly have reaction mechanism based on the detected attention levels in the individuals. To develop such attention aware system's, researchers from cognitive psychology, computer science, sociology, and other disciplines have shown collaborative efforts towards shared research agendas. Since most attention aware systems are developed based on application specific needs, it can be easier to categorize such research findings based on application areas. 


\subsection{Application specific need for attention aware systems}

There are several application areas where attention aware systems are being designed and developed; however, some of the major areas pertaining to such research have been described here.

\subsubsection{Human Computer Interface}

The exponential increase in digital computing systems and our growing dependency on it has redefined our today into what could be called as digital life. The presentation manner of these digital computing systems that ubiquitously surround us is becoming more and more important for improving human-computer interaction or HCI. Since the dawn of the digital age in the 1980's researches have been seeking new interaction methods and channels between humans and computers. For example, Bolt's ‘voice and gesture interface’ [39, 40], Wieser’s ‘ubiquitous computing’ [40], Jacobs ‘Eye Motion-based interaction' [41], and Nielsen's 'Non-command user interface' [42] are significant early attempts at developing successful computing environments. Pickard's et al. [43] research attempted to improve interaction between computers and humans through human emotions. The key aspiration behind such research was to increase the quality of productivity of humans using these computing systems and to inculcate prolonged periods of attention from the individuals towards the task being performed on the computing device.

Findings from discipline of cognitive psychology largely define how human attention plays in digital environments. Raskin's research [44] analyzed how single point 
of attention and habit formations has important relationship with an individual's ability to interact with computers. Abowd's [45] and Weiser's [40] research designed a wide range of systems with the aim of supporting the attention choices of the user by making the device "transparent" so that the user can focus on the task rather than the interface. Attentional Human Interface project at Microsoft research [46] is another notable research effort towards capturing the attention of users.

Some of the research and development of attention aware systems have closed loop feedback oriented designs, where the computer adapts based on the recognized affect state of the user. Lisetti et al. developed a tele-home health care system where various physiological and subjective components were used to diagnose different emotions and psychological status of the individuals [47]. Some studies have used facial features and facial expression of humans to understand and predict human emotion [48-51].

\subsubsection{Airline Industry}

The airline industry operates $24 / 7$ and sometimes, so do the folks who work in this industry. Recent reports on numerous errors by air traffic controllers and pilots alike have made the industry and its clients more sensitive to the increasing workload, erratic worktime schedules that the employees are forced to maintain. The US National Transportation Safety Board (NTSB) has identified fatigue as a contributor in more than 300 fatalities in airline accidents $[52,53]$. Moreover, the NTSB has identified fatigue as one of the 'Top Ten’ hazard list in its industry since 1990. Studies on air traffic controllers show that physiological and psychological responses (mood, physical fitness, fatigue) of these 
individuals are greatly affected based on varying workload and shift hours [52, 54]. Pilots and cabin crew are also highly susceptible to fatigue and loss of attention during flight operation, this could lead to disastrous implications, hence, counter measures such as Fatigue Avoidance Scheduling Tool (FAST) [55] and Psychomotor Vigilance Test (PVT) [56] etc. were developed.

\subsubsection{Road Traffic and Transportation}

A 2010 survey by American Automobile Association (AAA) [15] found that 41\% admit to have driven drowsy in the past. Drivers ages 16-24 were the most likely to report having fallen asleep while driving in the past year. The study also reports that one is six deadly road accidents and one in eight accidents with serious injury involved drowsy driving. Amongst all fatally injured drivers, it was determined that $92 \%$ of these accidents were simply because they were not paying attention to driving. More such alarming statistic are being reported each year worldwide. So with such a great need for transportation safety, several immediate measures need to be taken to reduce the cases of lack of attention and drowsy driving.

Several technologies are being developed for detecting drowsy drivers and helping them stay awake. Several crash avoidance technologies are being provided with modern cars such as brake assistance, lane departure warning, and blind spot detection. Mercedes and other top car manufacturers are developing their own 'attention detection technologies' as standard inputs to their cars, these technologies usually include, steering monitoring systems, braking monitoring, acceleration, time on road and road condition to recognize 
affect states of its drivers. Even the trucking industry is trying to adopt newer technologies to combat drowsy and fatigued truck drivers. Some of the new technology under developed also use video based driver monitoring or eye tracking technologies which monitor and determine the conditions of the driver such as, Smart Eye AB, Seeing Machines Face LA, Delphi Electronics Driver state monitoring, etc. However the fundamental problem with such face and eye tracking based monitoring systems is that it relies of various factors such as face visibility, ambient light, eyes visibility (sunglasses cannot be used.) etc.

\subsubsection{Learning environments and Learning Disorder}

Learning environments and modes of teaching have changed considerable in the past few decades. More and more technology is being embraced by student and teachers alike to try and improve the modes and delivery methods of content to the users. Prolonged attention spans and higher levels of cognition are vital for achieving maximum knowledge absorption and retention. However these are interpersonal qualities that vary tremendously from person to person. So attention aware system can help recognize individual patterns and stimuli requirement to achieve maximum affective learning. Within the learning systems itself, services which support attention aware processes are being developed based on certain key fundaments. Factual attention related information is physical observations by monitoring the physical interaction of the user with the learning environment. Information from observing keyboard strikes, mouse movements, eye tracking, speech detectors, etc. are being used for developing attention aware systems for education [57, 
58]. Some researchers are using psycho-physiological observations to during learning to determine psyco-physiological correlations, which happens to be similar to this research. Heart Rate Variability - HRV, Electroencephalogram EEG [59]etc have been used in the research to develop tools for measuring user engagement during learning task. Some tools are developed based on application observations which collects information based on how an application is used, such as capturing browsing history, pauses during reading, page progress etc. Finally user feedback and user direct input systems allows users to directly inform the system their affective learning states, desired manipulation of input and output mechanisms, etc. whereby, the system adapts itself based on these user inputs.

Attention Deficit Hyperactive Disorder (ADHD) and other disorders in children and adults are very prevalent and are being more recognized as a form of learning disorder around the world. For quite some time these disorders were being diagnosed trough behavioral observation. Recently more steps are being taken to standardize the diagnosis and treatment of such disorders. Technology such as neurofeedback and bio-feedback based on EEG, are being developed for an integrated approach towards detecting and improving the conditions of such disorders [60-62]. Biofeedback monitoring has resulted in effective methodologies to reduce interruption and limit focus on unrelated activities for ADHD patients, leading to improved performance of spelling and arithmetic tasks [63]. A computer-based system called BrightStar has also been developed which collects heartbeats and converts them to visual biofeedback to stimulate the brain and improve attention. 


\subsection{Significance of this research}

The common issue with attention detection technologies in different areas of applications is that it can be mostly used in clinical or standardized environmental settings and is not portable in most cases. Thereby, limiting the continual or daily study of patients or individuals in real world settings, which is required for a wholesome evaluation of patterns and motifs within individuals. Also most systems are based on non- physiological data or use a single physiological modal of input for attention recognition of the individual. The most popular of these modals for analysis is the EEG for recognizing alertness and drowsiness in individuals. EEG has shown tremendous success in speedily and accurately recognizing individuals affect state and cognition functionalities. EEG as a physiological modal as been studied tremendously and also has led to some significant developments in the field of cognition and neuro-psychology.

However, this proposed research stands out in comparison with existing ones since it has been fundamentally designed to be a multi-modal physiological signal based attention recognition system. It incorporates various signals such as the Electrocardiogram (ECG), Galvanic Skin Response (GSR), Heat Flux (HF), Skin Temperature (ST) etc. Extensive literature review reveals ECG signal has rarely been utilized in the development of such attention aware systems. Those studies that do use ECG only use HRV or hear rate variability as the most significant information. Hence this research attempts to use ECG as a significant modal for identifying affective attention states in humans. Here apart from 
HRV calculations several other mechanisms are used to derive useful features and information from the ECG signal for classification and development of a machine learning model for detecting existence of attention and the lack of it.

Another key significance of this research lies in the portable frame work that the process is being developed in. The crux of this study lies in being able to assess the corelationship between physiological signals and attention levels in the individual based on signals that can be collected using a commercially available portable armband. The processing of EEG signal collected in this study is to validate the results from the signals using the armband. 


\section{CHAPTER 3Experimental Setup and Description of Dataset}

\subsection{Overview}

A substantial aspect of this research has been the collection of the data itself. An extensive search revealed that there is no data set available, freely or otherwise, which cater to the exact needs to this particular study. Since the study is about utilizing the physiological signals collected via a portable armband for the detection of attention/focus or lack of it in an individual, the dataset had to be specifically catered for the requirements of this research.

Various kinds of experiments were designed and tested on test subjects to see which of them would be the best suited. The following were the criteria upon which these experiments were designed;

- Non Invasive: Since the data collection was on human subjects it was critical to ensure that their safety and comfort was given the highest priority. All the various physiological signals being collected were non invasive and were collected with commercially available and FDA approved devices.

- IRB regulated: The entirely experimental setup and data acquisition needs to be meticulously reviewed and approved by the Internal Review Board (IRB), of VCU's research office. 
- Similar Outcome: In view point of the subjects participating in the experiments, their experience and outcome of each portion of the experiment should be similar and standardized.

- Noise free: The data being collected should be relatively noise free. The environment in which the data collection was conducted needed to be controlled and ensures reduction in external anomaly and disruptions.

After testing with various designs of experiments the final design was chosen based on the satisfaction of all of the above criterions. The final design was developed under the helpful guidance of the psychologist involved with this research.

\subsection{Description of the conducted experiment}

For this study, the volunteer subjects were individually subjected to watching a series of pre-selected video clips. These videos clips are all freely available online, and were primarily chosen based on the content. All of the chosen clips fell in either of two categories that is either 'interesting' or 'non-interesting'. For each of these categories the respective sets of clips were concatenated to form a video montage lasting 20 minutes of viewing duration. Viewing the two set of videos one after the other, that is, 'Interesting' or 'non-interesting', required contrasting levels of engagement and focus from the participant. Ensuring (as far as possible) that the subjects were interested and paid attention to the interesting video set and the subjects were subsequently bored and lost focused attention during the non-interesting videos was the core concept behind the experiment. 
While each subject was subjected to viewing these video montages, physiological signals and video signals from the individual were collected. As mentioned the data collection was done under the consent of the IRB.

\subsection{Environment}

The experiment is conducted in an isolated room which is relatively dark with minimized distraction factors. The subject views the set of video on a laptop/desktop screen while the signal acquisition devices are kept behind them. The subject is in a seated position and is asked to move as little as possible during the entire experiment. They are briefed to look only at the screen in front of them showing the videos and nowhere else during the entire experiment. Once the subject is attached to the signal acquisition devices they are made to wait for 10 minutes before the experiment begins. This allows the signals such as GSR and HF to stabilize before the actual data collection begins. Timestamps are used to mark the beginning and end of each of the two sections in this experiment. 


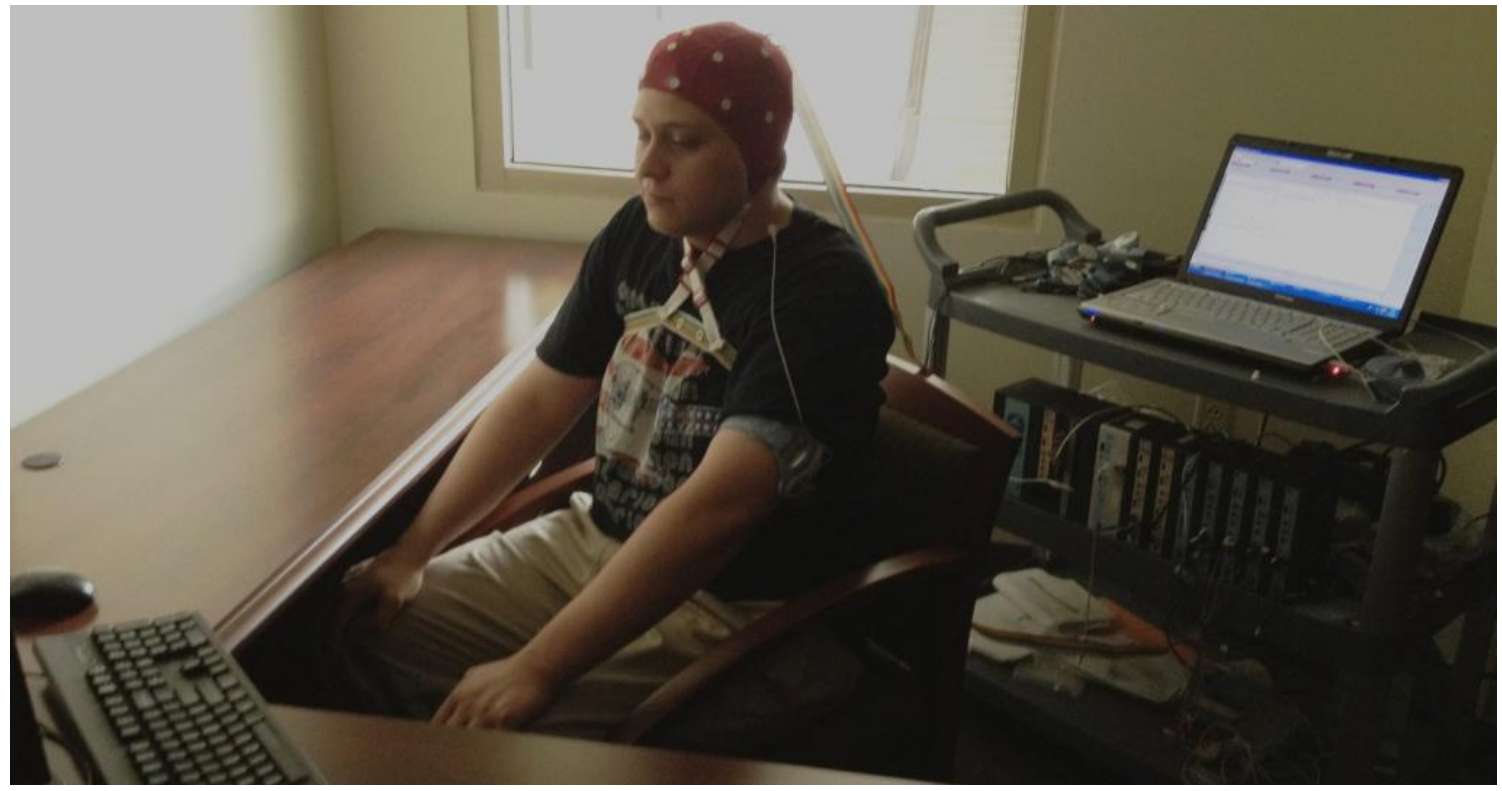

FIGURE 2. EXPERIMENTAL SETUP

\subsection{Physiological Signal Acquisition}

The various physiological signals acquired for this experiment are explained below.

\subsubsection{EEG}

The EEG was collected using the MP150 - EEG100C, a product of BioPack. With this system an EEG cap is provided that fits snug on the head of the subject and collects raw EEG signals in real-time. 

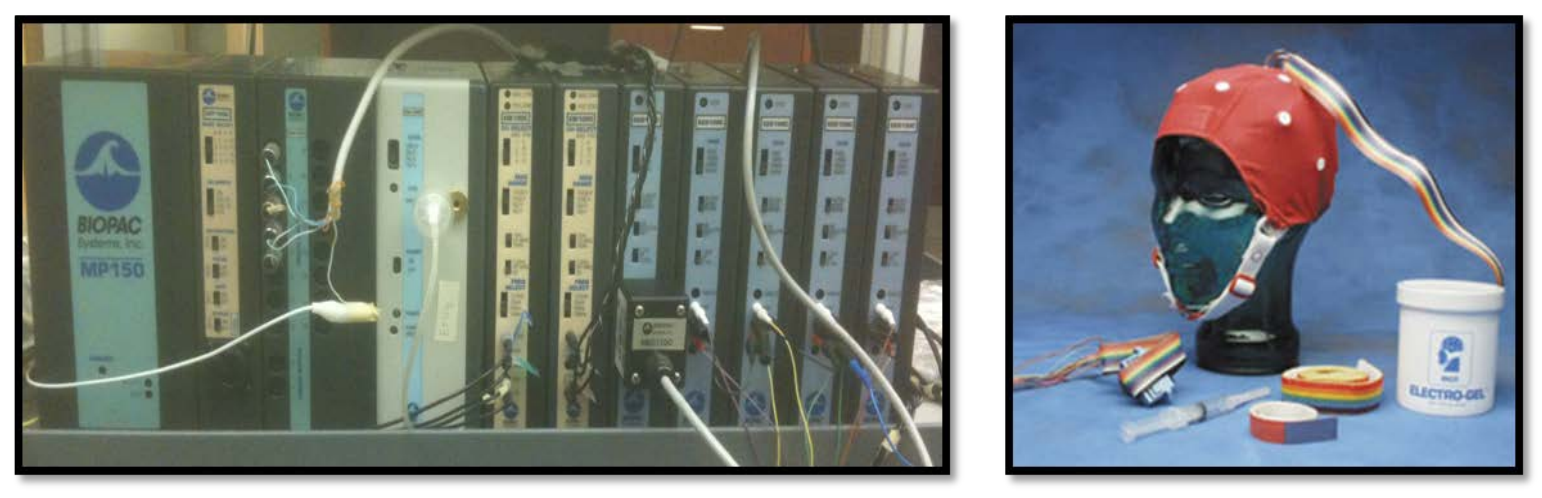

FIGURE 3. EEG ACQUISITION EQUIPMENT

The Lycra stretch cap holds nineteen imbedded tin electrodes closely to the subject's head.

Electrodes are pre-positioned in the international 10/20 format. Only skin contact is needed with the electrodes in the cap. When the electrode cap is in place, EEG recording gel is injected into each electrode (via a central gel access hole) with a blunt-tipped syringe. For the scope of this study, EEG data collected only from the frontal lobe (forehead placements) is used. The EEG signal is collected at $1000 \mathrm{~Hz}$.

\subsubsection{Armband Data:}

The SenseWear-Pro armband (a product of Body-Media Inc.) was used to collect other physiological signals. The SenseWear Armband is a versatile and reliable wearable body monitor worn on the upper arm and includes a 2-axis accelerometer, heat flux sensor, galvanic skin response sensor, skin temperature sensor, and a near-body ambient temperature sensor [64, 65]. The SenseWear armband is elastic and secured using Velcro. 
The armband is placed on the upper left arm of the subject for signal acquisition. The signals are acquired by the contacts on the armband and two ECG adhesive electrodes patch, which seats one on the arm and the other on the neck as shown in figure4. Only skin surface contacts are required and its small footprint on the body during use makes it ideal for portability and non-invasiveness.

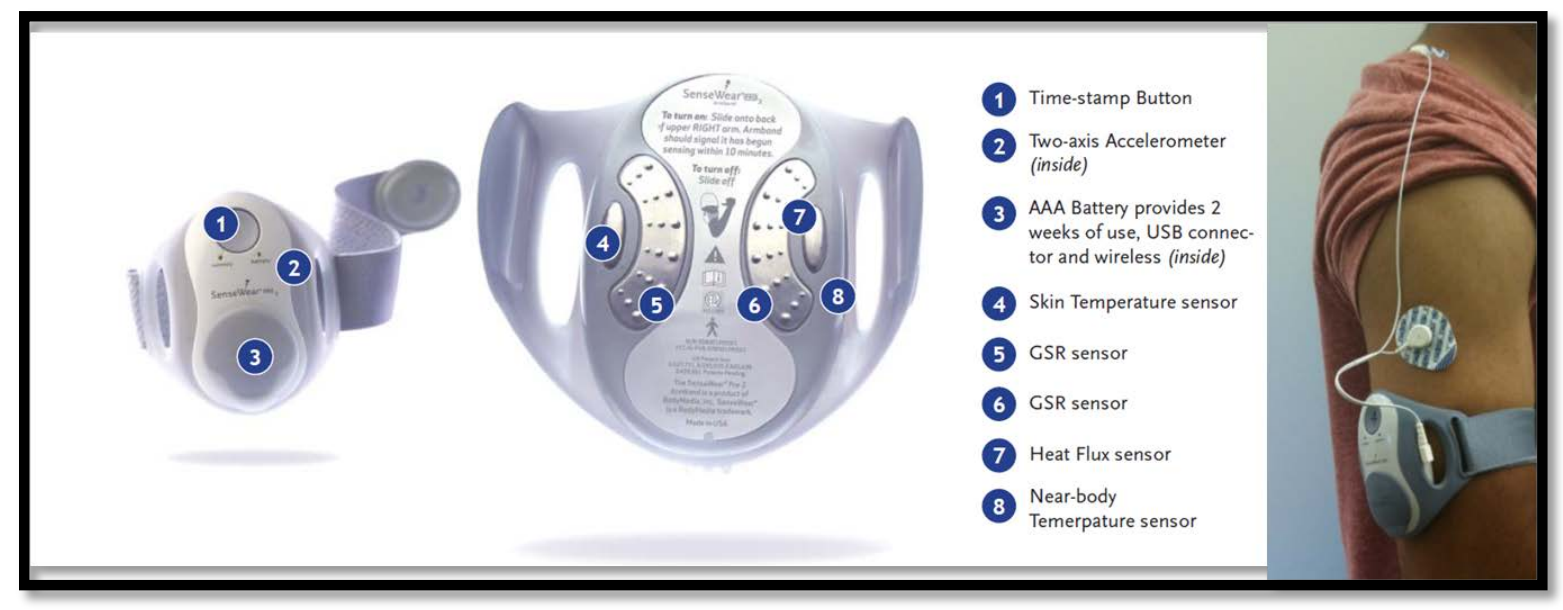

FIGURE 4. ARMBAND AND ITS SENSORS

The armband is capable of collecting ECG data at $128 \mathrm{~Hz}$. Al-Ahmad, Homer, and Wang [66] have presented preliminary results of validating these prototypes, showing that the armband measures heart rate and beat-to-beat variability comparably to a Holter monitor.

For this study the following signals collected from the armband:

- ECG -Electrocardiograph

- HF - Heat Flux

- GSR - Galvanic Skin Response 


\subsubsection{Video Signals}

An off the shelf web camera was used to record a video of the subjects face during the entire experiment. In this case the web-camera used is a Logitech Orbit camera. The intention of recording the subject's face is two folds; firstly it is to process the video using a facial feature recognition code which is able to identify the relative position of different aspects of the face such as eyebrows, eyes, blinks, nose etc. Converting these positions and movements into a feature space, allows us to study the various types of movements that are typically associated with focus and engagement as opposed to those associated with boredom and drowsiness. The second intention being that this video will allow the psychologist to validate the outcome of all the processing. The video signals collected for this research study are planned to be used as a part of the future work.

All data acquired for this research from the subjects will be entirely discarded after it is no longer need for the research. In all aspects, the person's identity and personal information which may have been acquired during data collection will be treated with complete discretion and will be securely maintained by the research team until it is in use. After which all the acquired data signals will be discarded properly. 


\section{CHAPTER 4 Methodology Overview}

\subsection{Introduction}

There are two general reasons for performing signal processing: (1) to acquire and process signals to extract and ascertain the existence of previously known information; and (2) for understanding the change in outcome and causal affect on a particular observation of a signal due to the presence of an external physical process. This research deals fundamentally with the latter aspect of signal processing.

The physiological signals that are being collected for this research are in essence time series. A time series is nothing but a collection of observations made sequentially in time. Other example of time series signals are ambient temperature data, seismic activity data, particulate air pollution measurements, etc. Much like how most process in nature are non-stationary, bio-signals are similar in that there is no systematic trend, no systematic change in variance and are not strictly periodic. Analysis and processing of time series data, in particular physiological data is not new science. There are countless methods and scientific approaches to choose from and apply towards analysis of the signals being collected as a part of this study. However the methods applied here are specifically chosen to analyze, understand and predict the possible changes that can be detected based on the effect a person’s attention, focus and engagement has on his/her physiological signal. 


\subsection{Methodology Background}

Just like static data or time series data clustering and classification, physiological signal classification requires a procedure to form clusters given a set of data objects and the choice of procedure depends both on the type of data available and on the particular purpose of application. As far as physiological signals are concerned, it is important to establish the nature of the distinctions that can made whether the signal is discrete-valued or real-valued, uniformly or non-uniformly sampled, univariate or multivariate, and whether data series are of equal or unequal length. Non-uniformly sampled data must be converted into uniformed data before clustering operations can be performed. Here all data is collected using uniform sampling.

Following is a broad generalization of various approaches one can take in analysis of physiological signals (or any time series signal for that matter).

\subsubsection{Raw Based Approach}

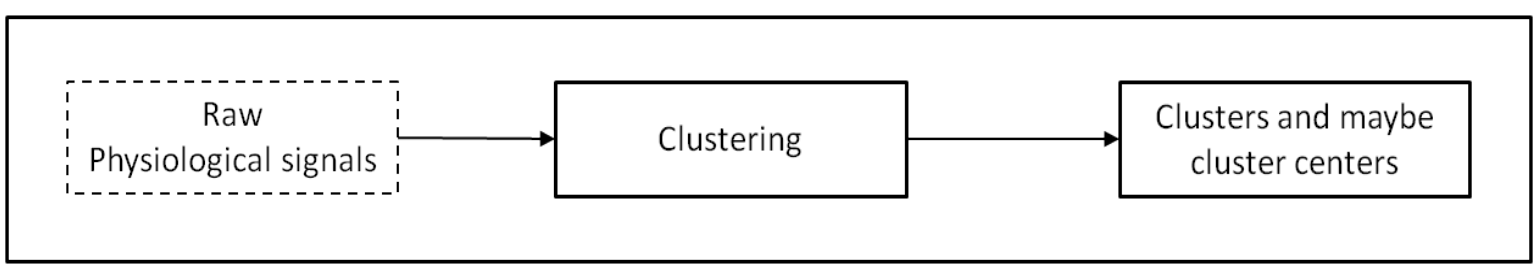

FIGURE 5. RAW BASED SIGNAL ANALYSIS APPROACH

The raw data based approach usually deals with treating the time series signal as static data and by using appropriate distance or similarity measures to form clusters. In this approach the formed clusters are analyzed for distinction within clusters and/or identifiable cluster centers to be able to develop means of classification of the original raw signals. 
With the perspective of the physiological signals in this study i.e. ECG, EEG, GSR, HF, the hypothesis of finding distinction in the signals between when a person is attentive or not, by using only the raw data is not very helpful. This raw signal based approach is too simple to identify and extract the complex yet subtle changes in the signal one expects with such a study. Clustering based on raw data implies working with high dimensional spaceespecially for data collected at fast sampling rates. Hence in this research methodology a more complex approach is employed to derive the necessary solution.

\subsubsection{Feature Based Approach}

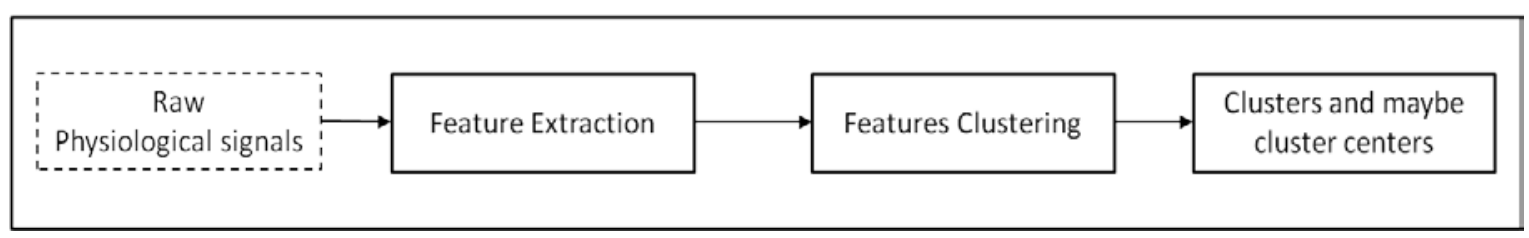

FIGURE 6. FEATURE BASED SIGNAL ANALYSIS APPROACH

It is important to note that while dealing with data with noise, as in the case of the physiological signals in this study, it is not desirable to work directly with the raw data. For this reason extracting features from the raw data and using the features for analysis, ameliorates the inherent undesirable effects. Though most feature extraction methods are generic in nature, the extracted features are usually application dependent. That is, one set of features that work well on one application might not be relevant to another. Some studies even take another feature selection step to further reduce the number of feature dimensions after feature extraction. 
This study has recognized that for signals such as HF, GSR and to an extent ECG as well, this approach for identifying distinction between different cases has been very useful.

\subsubsection{Decomposition Based Approach}

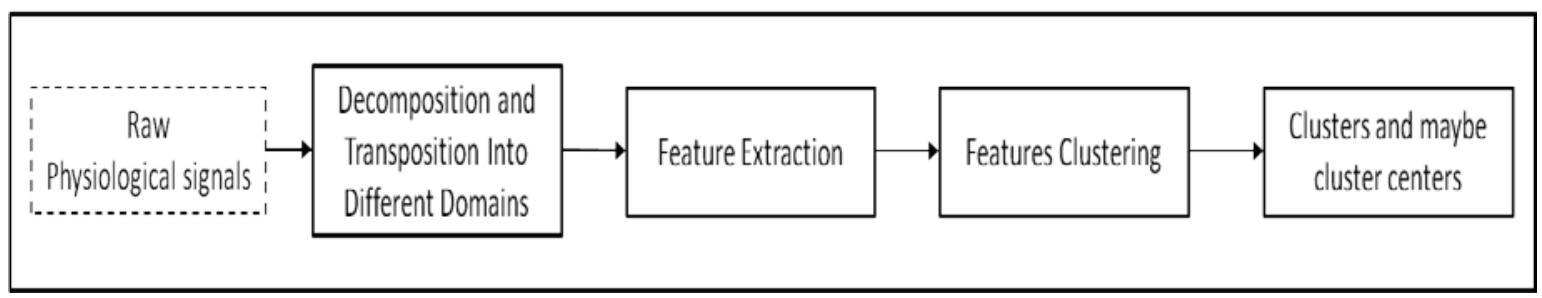

FIGURE 7. DECOMPOSITION BASED SIGNAL ANALYSIS APPROACH

Similar to how digital signal processing of time series data is performed in different domains, physiological signals can also be transposed to different domains such time domain, spatial domain, frequency domain, etc, or a combination of them for better analysis. The physiological signals in its original domain, i.e. time domain, may not be informative enough to reveal distinction and identifiable characteristics for the study's purpose for recognizing the presence or the lack of attention and engagement in the subjects. Hence in this research, sophisticated transformations methods have been employed to transform these signals into different domains which best represent the essential characteristics of the signal. 


\subsubsection{Model Based approach}

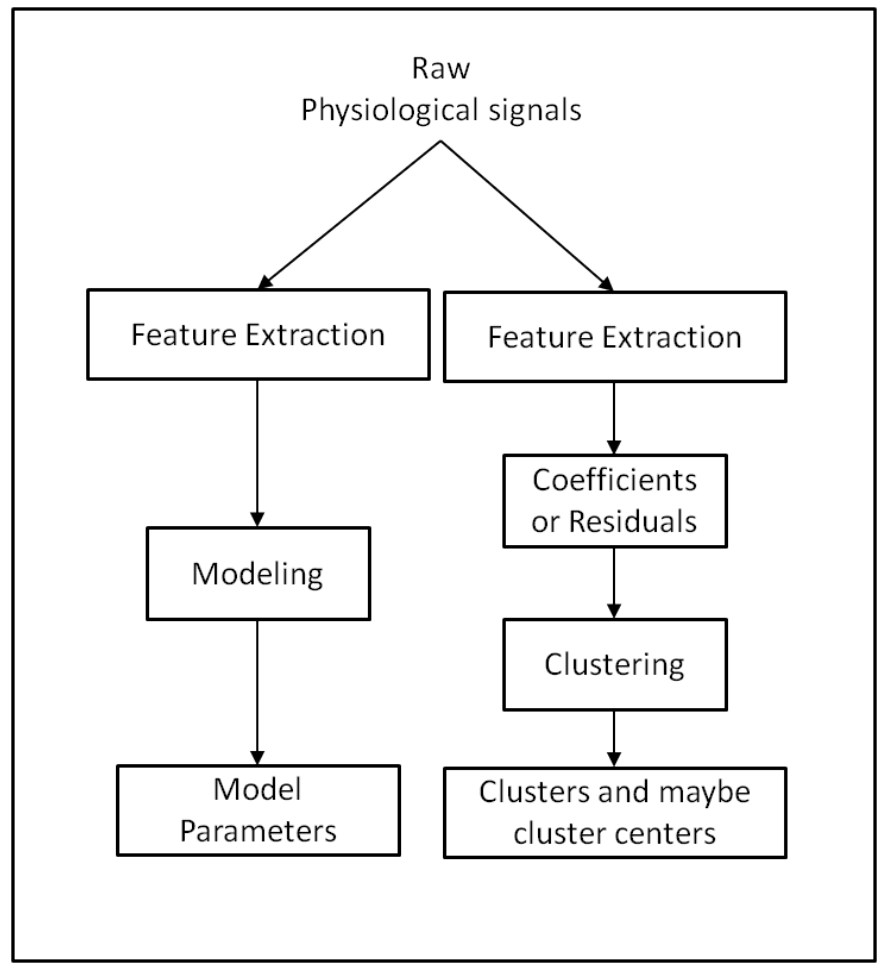

FIGURE 8. MODEL BASED SIGNAL ANALYSIS APPROACH

In the model based approach it is usually considered that the signal under observation is generated by some kind of model or by mixture of underlying probability distributions. Two independent cases of signals are considered similar if the models that characterize their respective signals or the remainder residuals obtained from each model after fitting is similar in nature. In this research the signals being collected under the two different conditions of attention and non-attention essentially are very similar in nature. It is the subtle hypothesized differences between these similar signals that need to be identified for this study. To do so, the complexity and sophistication of using model based 
approach increases tremendously and is something that might possibly be attempted in the future work of this research.

\subsection{Research Methodology - a Bird's Eye View Perspective}

The overall goal of this study is to be able to identify the engagement, attention and focus

of a person using only his/her physiological signals. Since the experiment involves the collection of various types of signals, each type of signal is analyzed in a different manner. The analysis methods chosen for each signal is catered and designed to best suit the inherent characteristics of the respective signal. The detailed description of each analysis has been presented later in the content of this proposal.

Here the overview and a global perspective of the entire research have been presented. 


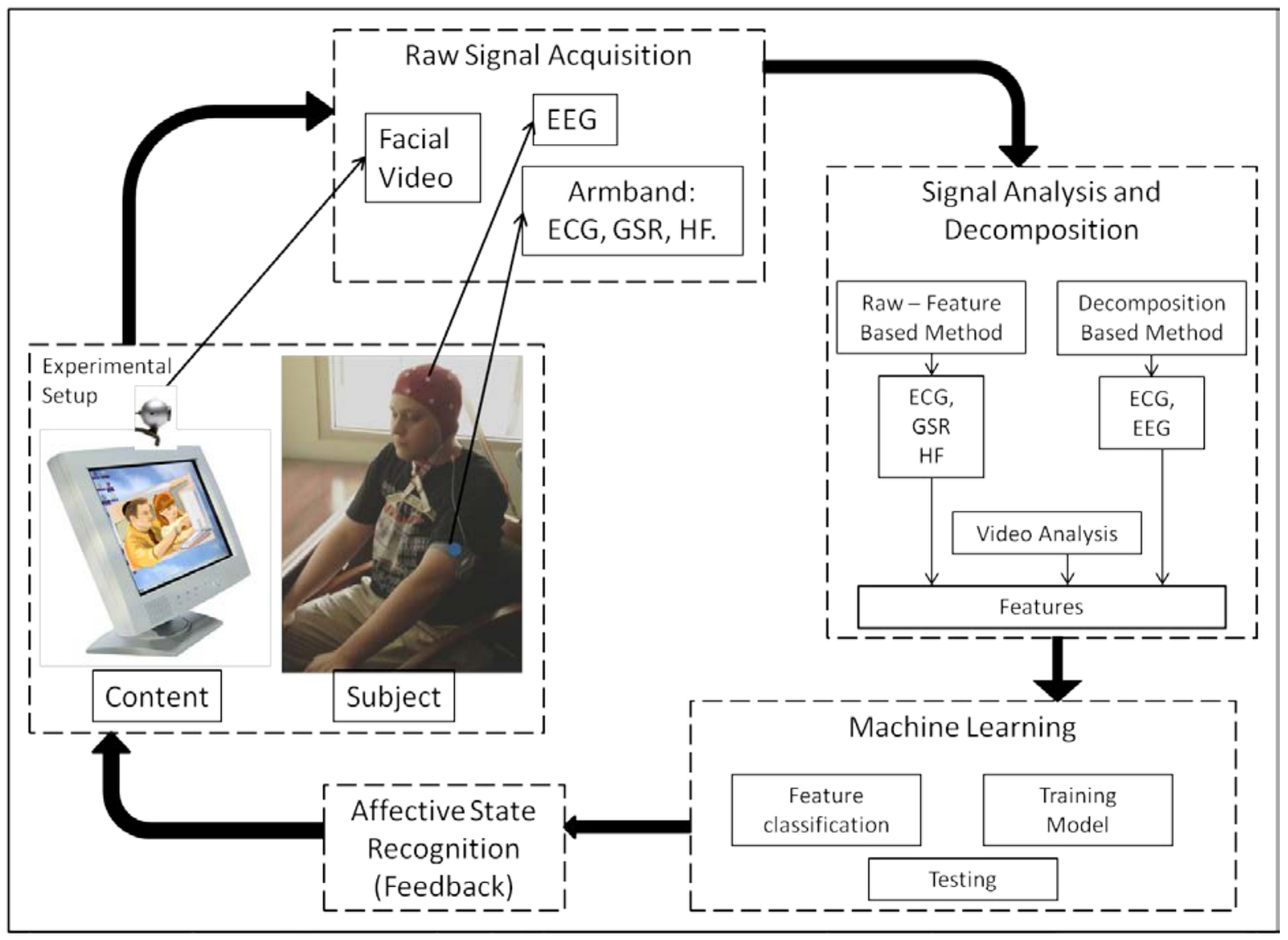

FIGURE 9. RESEARCH METHODOLOGY OVERVIEW

As shown in the above figure, the raw physiological signals as well as facial video is acquired from the subject as s/he views the 'interesting' and the 'non-interesting' videos. These signals are then analyzed and decomposed using some of the afore mentioned approaches.

It is to be noted that the overview diagram is a global perspective of the direction and aspirations of this study in its entirety, hence it shows the incorporation of GSR, HF signals as well as Video in the analysis and feature extraction stages. However, for the scope of this dissertation, results of only ECG and EEG analysis have been presented, 
while the analysis algorithms for the remaining signals are under various stages of development and testing.

After the physiological and video signals have been analyzed and decomposed, relevant and useful features are extracted and fed to the next stage which is machine learning and classification.

In the machine learning stage using feature sets from several previous subject, a classification model is computed which is used to test and classify the signals features from the current subject. The classification of the current acquired and processed physiological signal would fall under one of two categories; either 'Interesting' or 'Noninteresting'. This real-time classification facilitates the recognition of the 'Affect State' of the subjects' attention / focus levels and thereby predicts the engagement of the subject with the presented content.

The ability to quantize or enumerate a person's engagement level can be of vital importance, especially in the feedback stage. The feedback here is highly situation dependent and the feedback system must be customized for each specific application area. For example, if a person is driving an automobile and the system predicts low engagement levels and thereby recognizes lack of focus / attention in that individual, the systems feedback mechanism could alert the person by making a loud noise or announce instruction over the speakers in the car. It could also redirect the GPS to the nearest coffee shop before continuing to the destination. 
It is also to be noted that since the feedback is highly situation and application dependant, the affective state recognition, quantization and feedback mechanisms are a part of the future work of this research. 


\section{CHAPTER 5 Analysis of Electrocardiogram}

\subsection{Overview}

Electrocardiograph which is more popularly known as ECG or EKG (from the Dutch Electrokardiogram) is a physiological signal which comprises of tiny electrical discharges of the heart. This electrical signal is usually captured from the skin when the heart muscles depolarizes during each heartbeat. The idea of studying the ECG for detecting ailment has been around for little over a century now. Although the discovery of these electrical activities of the heart was discovered by Alexander Muirhead in 1872, it was not until 1903 when Willem Einthoven from the Netherlands created a breakthrough in naming the waves in the ECG and applying it towards medical sciences. For which he was awarded the Nobel Prize in Medicine (1924).

Historically, ECG has been extensively used for monitoring vital signs and diagnosing cardiac disorders of a patient. The ECG is a realistic record of the direction and magnitude of the electrical activity that is generated by depolarization and re-polarization of the atria and ventricles of the heart. The figure below depicts a cardiac cycle in an ECG signal which consists of the P-QRS-T waves. Within the intervals and amplitudes defined by the cardiac cycle lies majority of the clinically useful information [67]. A cardiac cycle of the ECG is also referred to as the sinus rhythm, since it is repeatedly occurs during each 
heart beat. Figure 10(a) shows a portion of the ECG from the collection in this study, figure 10(b) describes the significant points in the cardiac rhythm.

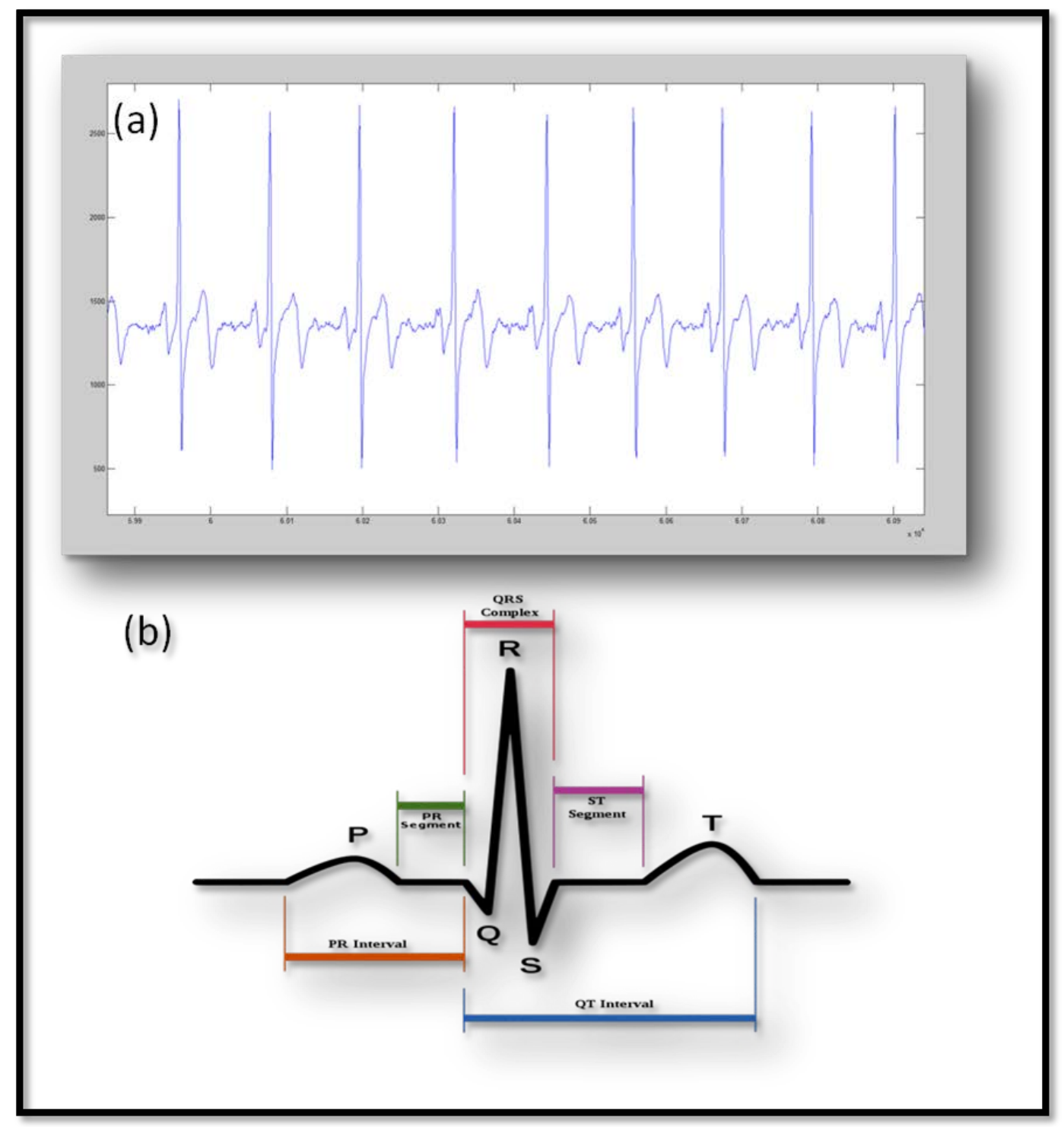

FIGURE 10. (A) ECG SIGNAL, (B) CARDIAC COMPLEX

Over the years researchers and physicians have developed several ways of analyzing the ECG signal [68-70]. Typically, cardiac cells in the normal state are electrically polarized the deviations in the normal electrical patterns indicate various 
cardiac disorders [71]. Hence based on this fact, a typical ECG analysis system extracts the fundamental features of the sinus rhythm (amplitude and intervals).

Physicians and some earlier methods of ECG analysis usually performed analysis only in the time domain. This might have been because they didn't have the means to automatically analyze the real-time recorded ECG with computer aided systems. Although analysis in the time domain alone has been proven useful, effective and is still practiced, time domain alone cannot provide certain subtle information which might be inherent within the signal. Especially considering the case of this research, the ECG collected is from healthy individuals during two stages of simple testing, i.e. during a phase of attention and a phase of lack of attention.

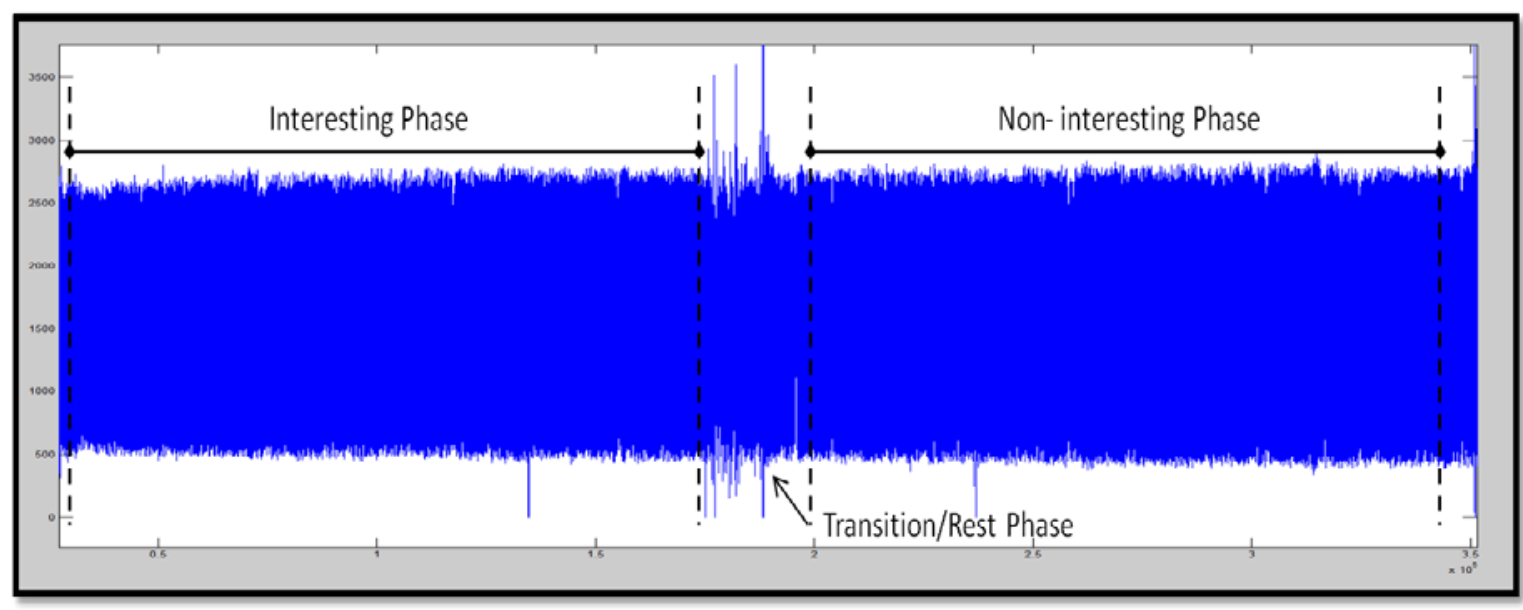

FIGURE 11. ECG SIGNAL FROM A SUBJECT

As shown in the figure11, visually the ECG collected from the same individual during both the test phase look strikingly similar. Hence to be able to find certain characteristic differences between the two cases of ECG, in this research, the analysis of the collected ECG has been performed in various domains and not limited to the time domain alone. 


\subsection{Analysis Methods}

The flowchart in figure12 below shows the procedure of analysis implemented in this project so far. It begins with the acquisition of raw ECG signal from the subject while the individual watches the two sets of video montages (interesting and non-interesting). As with most physiological signals after the raw signal has been collected, some preprocessing might to be performed on the signal before the signal can be analyzed further. After preprocessing the ECG signal is analyzed with two of the earlier described fundamental approaches, i.e. raw signal to feature based approach and decomposition based approach.

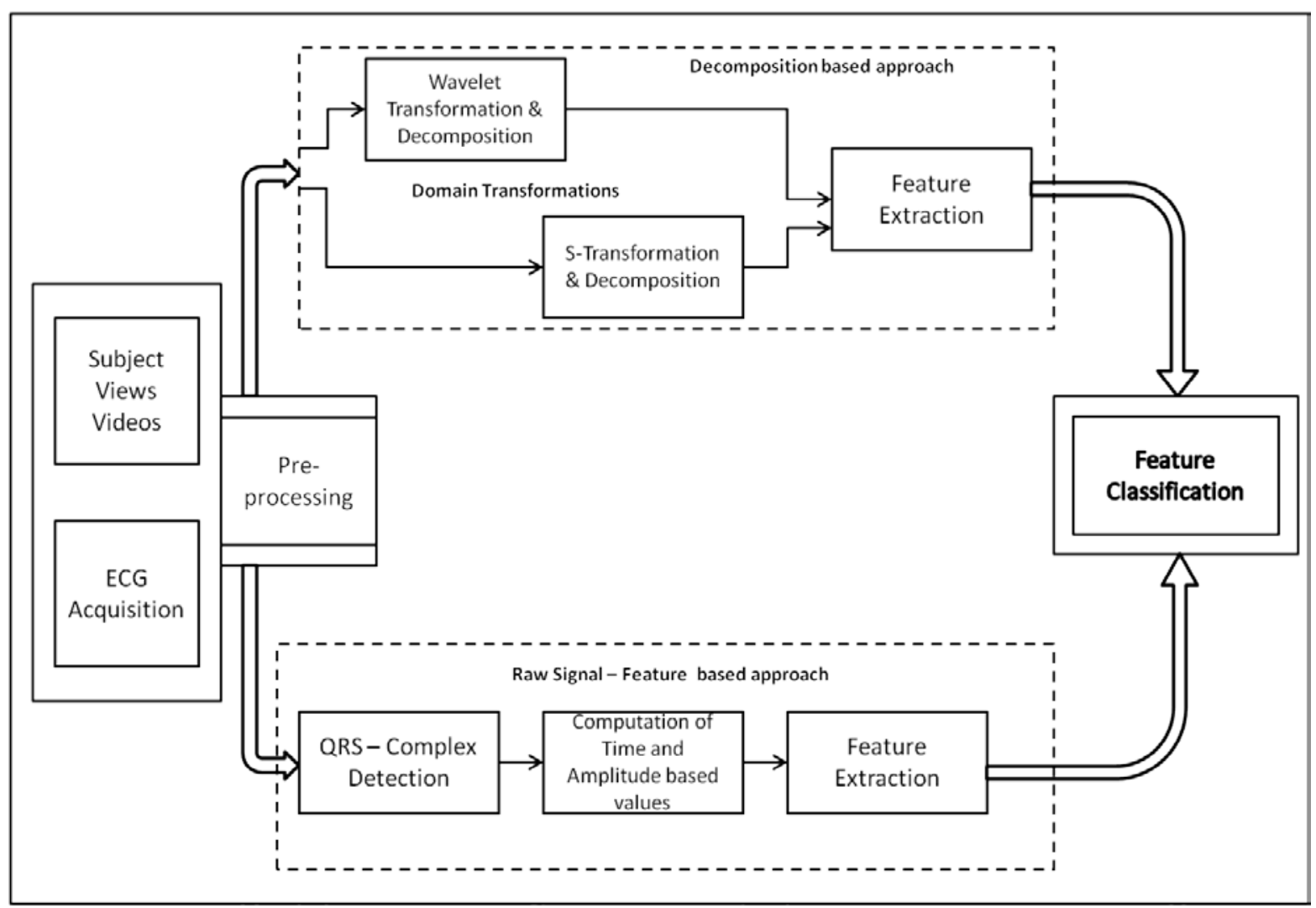

FIGURE 12. ANALYSIS IMPLEMENTATION PROCEDURE 
In the raw signal to feature based approach, the QRS complex of every detected heart wave is identified and extracted. Using each successive identified QRS complex certain time and amplitude based values are extracted. Using these values, certain statistical features are computed which are then fed into the machine learning phase of the analysis.

In the decomposition based approach, the pre-processed ECG signal is decomposed using two different domain transform methods. The first method is the 'Wavelet Transform' method which transforms and decomposes the signal into the wavelet domain thus facilitating the extraction of certain key features. The second method being the Stransform method, here 'S' stands for 'Stockwell' named after its inventor. After performing S-transform on the signal more features are extracted from the signal which is combined with all the other extracted features and then classified using machine learning techniques. Next the analysis methods and application have been explained in more detail.

\subsection{Preprocessing of ECG}

The acquired raw ECG signal contains some inherent unwanted artifacts that need to be dealt with before any analysis can be performed. The cause of these artifacts, which is usually frequency noise or baseline trend, could be due to a number of reasons such as subjects' movement causing motion artifacts, breathing patter artifact, loose skin contact of the electrodes, electric interference (usually found around $55 \mathrm{~Hz}$ ) etc. Therefore a pre-

processing step has been designed to ensure that the signal is as clean and artifact free before analysis. 


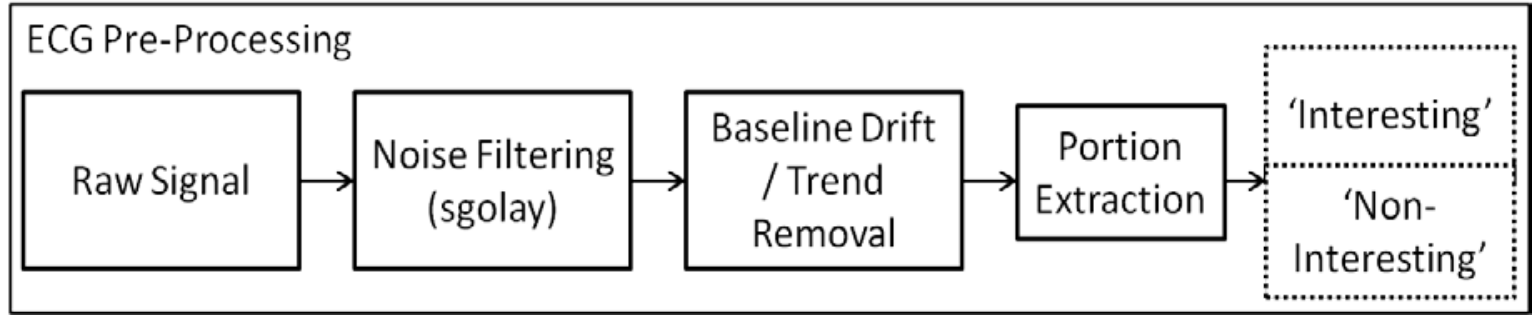

FIGURE 13. ECG SIGNAL PREPROCESSING

\subsubsection{Noise Removal}

As shown in the chart above (figure13), the raw ECG signal is first sent through a noise removal step. Noise removal in essence is filtering the signal to remove unwanted frequencies and retain only useful information. Since each signal has to be filtered differently based on the type inherent noise, for the ECG signal 'SGolay' filtering method has proven to be an adequate filter. The 'SGolay' stands for Savitzky-Golay filter. This filter is a digital polynomial filter based on least square smoothing mechanism. The SGolay filters are typically used to smooth out a noisy signal with a large frequency span. They perform better than standard averaging FIR filters, since these filters tend to retain a significant portion of the signals high frequency content while removing only the noise [72]. The application and performance of Savitzky-Golay filter on ECG Signal has been described in [73]. 


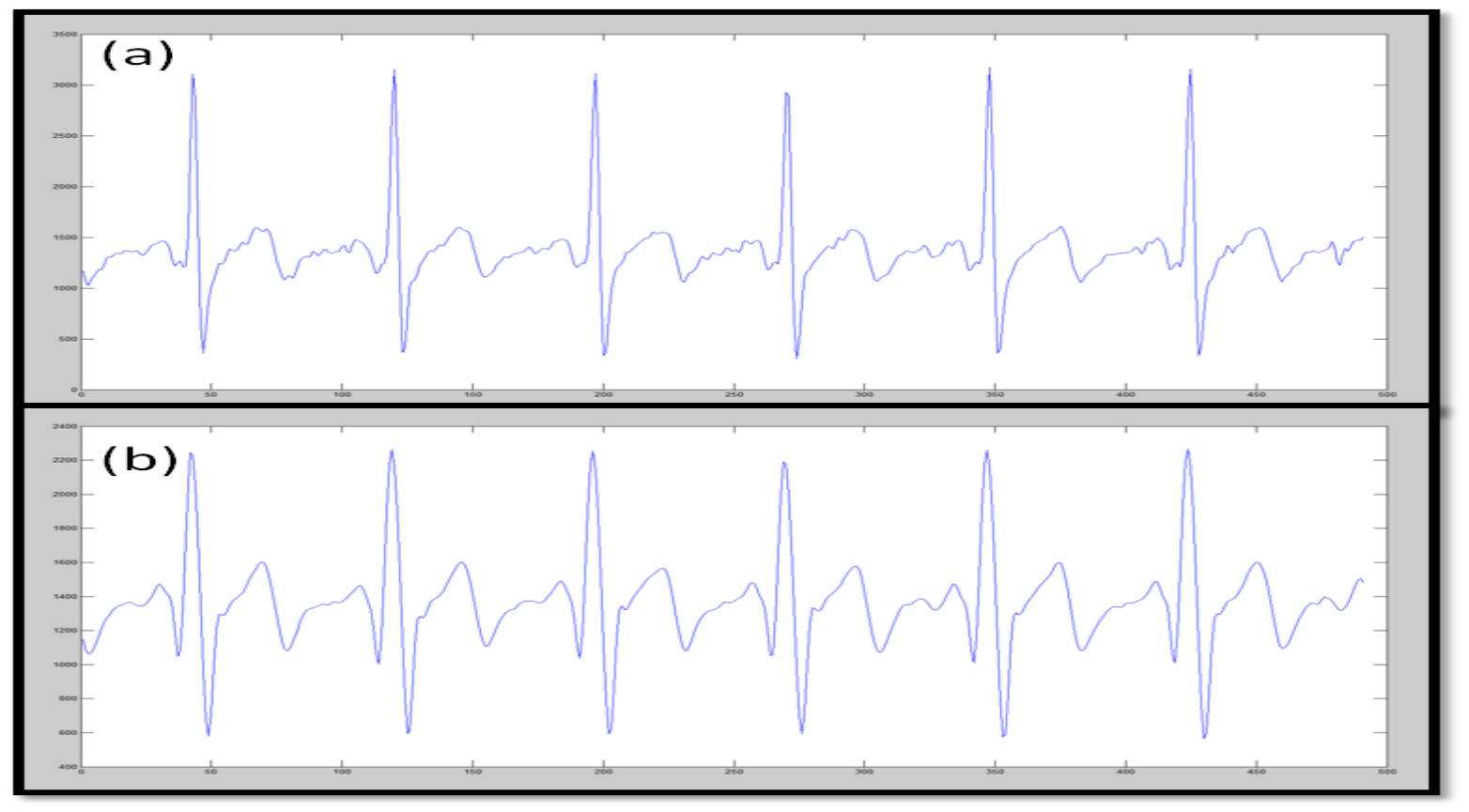

FIGURE 14. (A) BEFORE NOISE REMOVAL, (B) AFTER NOISE REMOVAL

The figure 14(a) shows the signal before filtering and 14(b) shows the signal after filtering. It can be seen that sgolay filter successfully removed the high frequency distortion that was present in the original signal

\subsubsection{Baseline Drift Removal}

Some of the most significant reasons for baseline drift in ECG recordings are due to respiration, muscle contraction, and electrode impedance changes due to subject's movement [74]. Baseline drift in the ECG signal alters the true amplitude of the sinus rhythm values and thereby it can influence the features computed, affecting the output of the entire system. 


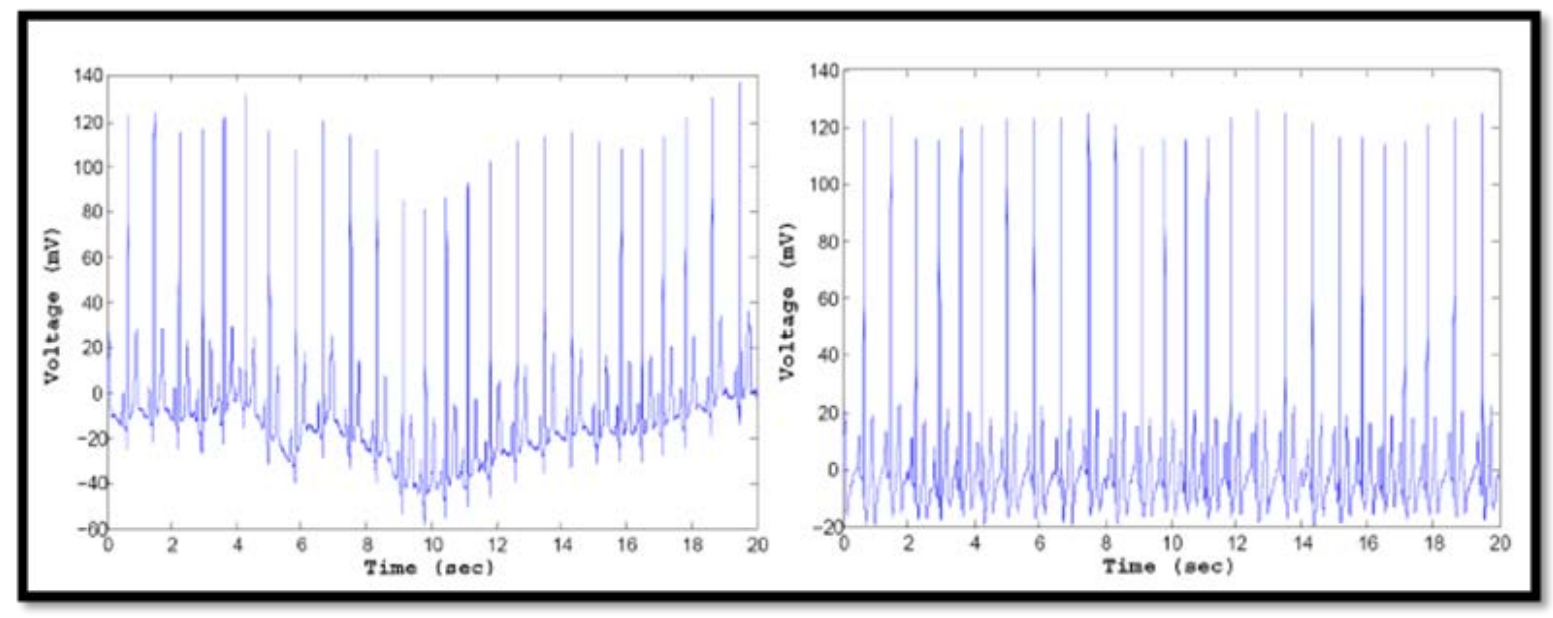

FIGURE 15. ECG BASELINE DRIFT REMOVAL, BEFORE AND AFTER

The method adopted to remove the baseline drift first determines the regression line that best fits the samples within a window of size equal to the sampling rate. Since the sampling rate of the acquired ECG signal is $128 \mathrm{~Hz}$, a one second window inside the signal will contain 128 points. Next the computer regression line is subtracted from the original signal window and thereby eliminating the drift.

Given $n$ points of the ECG signal $\left(x_{1}, y_{1}\right),\left(x_{2}, y_{2}\right), \ldots,\left(x_{n}, y_{n}\right)$, the best fit line associated with these points can be computed as follows:

$$
\begin{gathered}
m=\frac{n\left(\sum_{1}^{n} x y\right)-\left(\sum_{1}^{n} x\right)\left(\sum_{1}^{n} y\right)}{n\left(\sum_{1}^{n} x^{2}\right)-\left(\sum_{1}^{n} x\right)^{2}} \\
b=\frac{\sum_{1}^{n} y-m\left(\sum_{1}^{n} x\right)}{n} \\
y=m x+b
\end{gathered}
$$


Where $y$ is a point on the line, $m$ is the slope of the line and $b$ is the intercept. The computed best fit line for each window is then subtracted from the original signal window to obtain a baseline drift free signal.

\subsubsection{Portion Extraction}

After ECG signal has been filtered of noise and baseline drift, the signal is then split into two important sections based on the acquisition and experiment framework. These two sections or portions are 'Interesting' and 'Non-Interesting', which is directly based on the two video montages that the subject views one after another. The two portions of signals are extracted from the original signal using the timestamps that are recorded and indexed at the beginning and end of each portion of the signal acquisition.

It is important to separate these two sections of signals at this stage simply because in the Machine Learning (ML) stage, the features extracted from these two sections of signals need to be classified accordingly. The classified and supervised feature sets also known as 'training-set' are only to be used during the learning phase of the ML. During the learning phase a model is developed which will then be utilized to categorize and classify fresh data set aka 'testing-set' which have not been bifurcated into 'interesting' and 'noninteresting' portions and hence are not supervised. 


\subsection{QRS Complex Detection}

As shown earlier the methodology overview figure, one of the methods employed in analyzing the ECG signal is raw signal to feature based approach. In this approach the signal after pre-processing is sent through a QRS complex detection method.

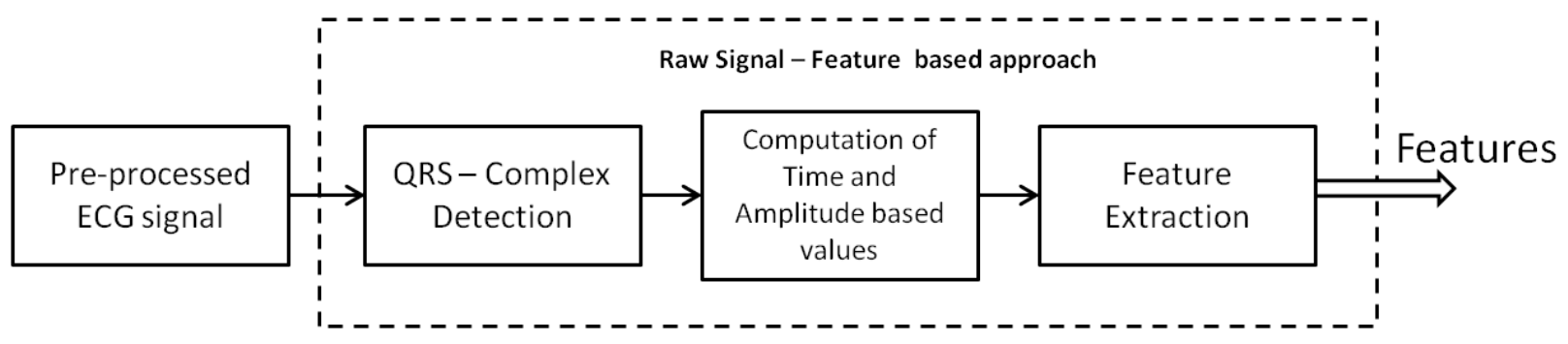

FIGURE 16. QRS COMPLEX DETECTION STEPS

Various QRS complex detection algorithms have been in development and use for decades now [75, 76]. Detection of QRS complex has been vital in clinical application and diagnosis since any irregularities detected in the sinus rhythm can be an indication of some underlying disease or disorders. Existing QRS complex detecting algorithms utilize methods such as Wavelet Transform [77-81], Artificial Neural Networks [82, 83], Mathematical Morphology [84-86] etc.

For this study, the QRS detection is performed using a novel algorithm developed by Yurong et al [87]. This QRS detection method was chosen for this study because, it had high detection accuracy as compared to other existing methods, it also minimized both false positives and missing peaks detection thus proving to be very useful for our study. In this method, Hilbert transform is applied to the pre-processed ECG signal to form a new signal in which the amplitude of the difference between the R wave and other waves, such 
as $\mathrm{P}$ wave and $\mathrm{T}$ wave, is greatly enhanced making QRS complex detection much more accurate. Also in case of the presence of inverted R waves, they are identified and thus solving the problem of missing any complexes. More details of how this method is implemented is given in [87]. However, for application of this algorithm with the dataset of this study, some modifications were made such as adaptation of the algorithm with 128HZ armband ECG etc.

After the pre-processed signal is processed with the QRS complex detection algorithm, certain key time and amplitude features are calculated from it.

\subsubsection{Time Based Values}

The figure below shows the detected QRS complex and the various time and amplitude based values that are to be computed from them. All values are computed inside 10 seconds windows of the original time series.

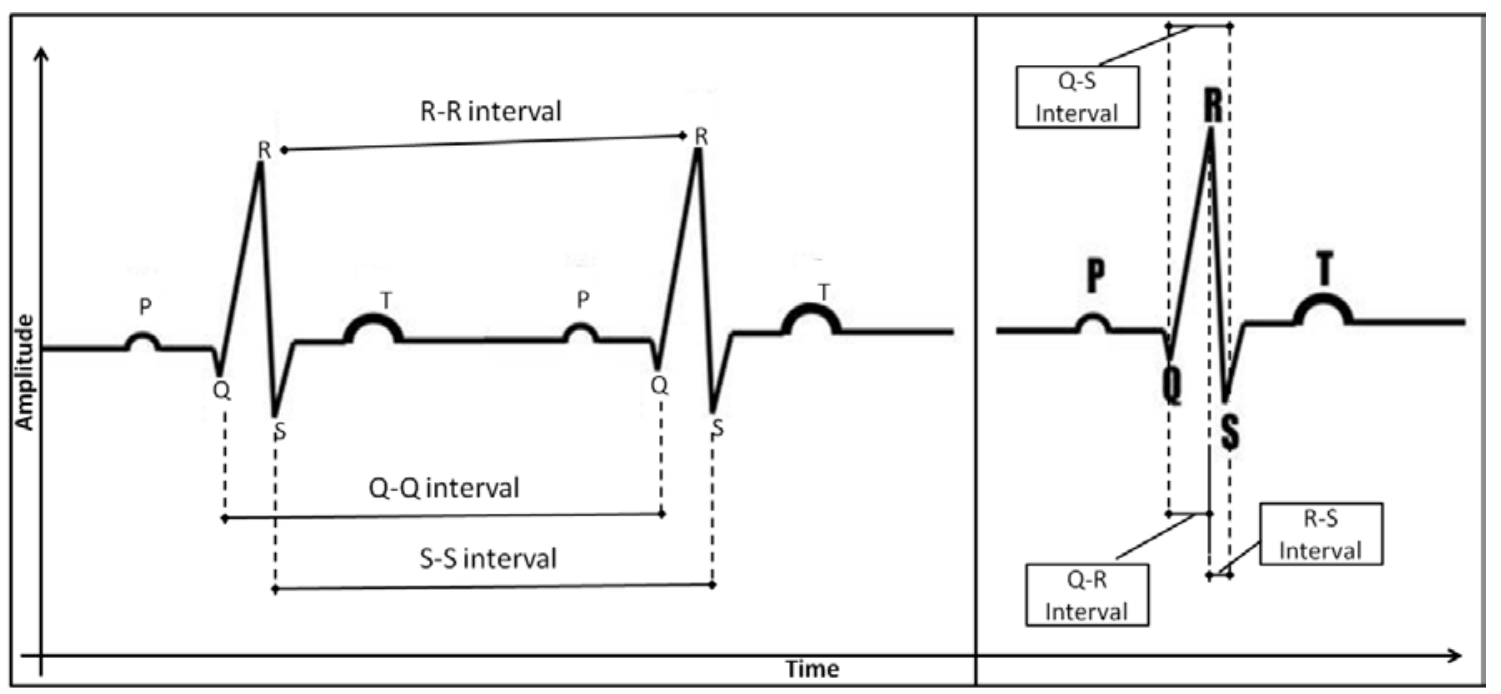

FIGURE 17. TIME BASED VALUES IN ECG 
For computing the values in time domain, the following formula is adopted:

$$
\text { int }=y-x ;(\text { time domain })
$$

Where $x$ is the first point of interest in time and $y$ is the next point of interest in time and int is the difference of the two time values, thereby giving us the interval between the two points of interest. In this manner, the following intervals are calculated:

$$
\begin{aligned}
& \text { R-R interval } \\
& \text { Q-Q interval } \\
& \text { S-S interval } \\
& \text { Q-S interval } \\
& \text { Q-R interval } \\
& \text { R-S interval }
\end{aligned}
$$

These values are further used in feature extraction step.

\subsubsection{Amplitude Based Values}

Using the amplitude information of the detected QRS complex, more values are computed. During pre-processing the steps of removing noise and baseline drift can sometimes affect the absolute amplitude values of the recorded ECG signal. Hence, to incorporate the amplitude values into the analysis, relative amplitude ratios of each significant point of the QRS complex is calculated. This is done as follows:

$$
a m p_{\text {ratio }}=\frac{x_{a m p}}{y_{a m p}}
$$


Where $x_{a m p}$ is the amplitude of the first point of interest, $y_{a m p}$ is the amplitude value of the second point of interest and $a m p_{\text {ratio }}$ is the ratio of the two points in the amplitude domain. Using this function the following amplitude based values are computed:

$$
\begin{aligned}
& \text { R-S amplitude ratio } \\
& \text { Q-R amplitude ratio } \\
& \text { Q-S amplitude ratio }
\end{aligned}
$$

These amplitude based values along with time based values are all used in the next step that is feature extraction.

\subsubsection{Feature Extraction}

With the computed time intervals and amplitude ratios of all detected QRS complex, feature extraction is then performed on these values. The values extraction is done inside windows of 10 seconds each. Hence the features computed are based on the values computed inside each 10 seconds window. The computed features are as follows:

For each set of values inside the 10 second window, $\mathrm{x}_{1}, \mathrm{x}_{2}, \ldots \mathrm{x}_{\mathrm{n}} \in \mathrm{X}$ (where $n$ is the number of elements in the window)

- Minimum

$$
\min =\operatorname{minimum}\left(x_{1}, x_{2}, \ldots, x_{n}\right)
$$

- Maximum

$$
\max =\operatorname{maximum}\left(x_{1}, x_{2}, \ldots, x_{n}\right)
$$


- Standard Deviation

$$
s t d=\frac{1}{n} \sum_{i=1}^{n} x_{i}^{2}
$$

- Window Mean

$$
\text { mean }=\frac{\sum_{i=1}^{n} x_{i}}{n}
$$

- Energy

$$
\text { energy }=\sum_{i=1}^{n}\left|x_{i}\right|^{2}
$$

- Power

$$
\text { power }=\frac{1}{n} \sum_{i=1}^{n}\left|x_{i}\right|^{2}
$$

\section{- Median}

The median is the middle value of a set of data containing an odd number of values, or the average of the two middle values of a set of data with an even number of values.

- Slope

$$
\text { slope }=\operatorname{mean}\left(\frac{\partial F}{\partial x}\right)
$$

Where $\left(\frac{\partial F}{\partial x}\right)$ corresponds to the difference in $x$ horizontal direction of

the susequent $x^{\prime} s$.

\section{- Skweness}

Skewness is a measure of the asymmetry of the data around the sample mean. If skewness is negative, the data are spread out more to the left of the mean than to 
the right. If skewness is positive, the data are spread out more to the right. The skewness of the normal distribution (or any perfectly symmetric distribution) is zero.

$$
\text { skewness }=\frac{\frac{1}{n} \sum_{i=1}^{n}\left(x_{i}-\bar{x}\right)^{3}}{\left[\sqrt{\frac{1}{n} \sum_{i=1}^{n}\left(x_{i}-\bar{x}\right)^{2}}\right]^{3}}
$$

\section{- Kurtosis}

Kurtosis is a measure of how outlier-prone a distribution is. The kurtosis of the normal distribution is 3. Distributions that are more outlier-prone than the normal distribution have kurtosis greater than 3; distributions that are less outlier-prone have kurtosis less than 3.

$$
\text { kurtosis }=\frac{\frac{1}{n} \sum_{i=1}^{n}\left(x_{i}-\bar{x}\right)^{4}}{\left[\frac{1}{n} \sum_{i=1}^{n}\left(x_{i}-\bar{x}\right)^{2}\right]^{2}}
$$

- Range

$$
\text { range }=\operatorname{maximum}\left(x_{1}, x_{2}, \ldots, x_{n}\right)-\operatorname{minimum}\left(x_{1}, x_{2}, \ldots, x_{n}\right)
$$

Let vector $\mathrm{D}$ represents the first order variations in $\mathrm{X}$, and vector $\mathrm{G}$ represents the second order variations such that:

$$
\begin{aligned}
& d_{i}=x_{i}-\mathrm{x}_{\mathrm{i}-1} \\
& g_{i}=d_{i}-\mathrm{d}_{\mathrm{i}-1}
\end{aligned}
$$


Now, using the signal vector $\mathrm{X}$ and vectors $\mathrm{D}$ and $\mathrm{G}$, the following first and second order factors are defined:

$$
\begin{gathered}
S_{0}=\sqrt{\frac{\sum_{i=1}^{n} x_{i}^{2}}{n}} \\
S_{1}=\sqrt{\frac{\sum_{i=2}^{n-1} d_{i}^{2}}{n-1}} \\
S_{2}=\sqrt{\frac{\sum_{i=3}^{n-2} g_{i}^{2}}{n-2}}
\end{gathered}
$$

Then the flowing features can be computed:

- Signal Complexity

$$
\text { Signal Complexity }=\sqrt{\frac{S_{2}^{2}}{S_{1}^{2}}-\frac{S_{1}^{2}}{S_{0}^{2}}}
$$

- Signal Mobility

$$
\text { Signal Complexity }=\frac{S_{1}}{S_{0}}
$$

\section{- Log of Variance}

Let the probability mass function be as each element be as follows $x_{1} \mapsto p_{1}, \ldots, x_{n} \mapsto p_{n}$, then

$$
\text { Variance }=\sum_{i=1}^{n} p_{i} *\left(x_{i}-\mu\right)^{2}
$$


Where, $\mu$ is the expected value, i.e.

$$
\mu=\sum_{i=1}^{n} p_{i} * x_{i}
$$

$$
\text { Therefore, } \quad \text { Log of variance }=\log _{2}(\operatorname{Variance}(x))
$$

- Mean of frequencies (Discrete Fourier domain)

$$
d f t\left(x_{k}\right)=\sum_{k=1}^{N-1} X(j) e^{j\left(\frac{2 \pi}{N}\right) k n}
$$

Where a net of $\mathrm{N}$ time samples, $d f t\left(x_{k}\right)$ represents the magnitude of sine and cosine

$$
\begin{aligned}
& \text { componenets in the samples given by } e^{j\left(\frac{2 \pi}{N}\right) k n} . \\
& \text { Hence, } \\
& \text { mean of fourier domain }=\text { mean }(\operatorname{dft}(x))
\end{aligned}
$$

- Variance of probability distribution

Probability Distribution Function $=P[a \leq x \leq b]=\int_{a}^{b} f(x) d x$

$$
\text { Variance of distribution }=\text { variance }(P)
$$

\section{- Sum of Auto-Correlation}

$$
\text { Autocorelation fucntion }=R(s, t)=\frac{E\left[\left(x_{t}-\mu\right) *\left(x_{t+r}-\mu\right)\right]}{\sigma_{t} \sigma_{s}}
$$

Where, $s$ and $t$ are different times in the time series, $\mu$ is the mean of $X, \sigma$ is the standard deviation of $\mathrm{X}$ and "E" is the expected value operator. 


$$
\text { Sum of AutoCorrelation }=\operatorname{sum}(R(s, t))
$$

\section{- Mean of Auto-Covariance}

$$
C(s, t)=E\left[\left(x_{t}-\mu_{t}\right) *\left(x_{s}-\mu_{s}\right)\right]
$$

Where, $\mathrm{s}$ and $\mathrm{t}$ are different times in the time series, $\mu$ is the mean of $\mathrm{X}$, and "E" is the expected value operator.

$$
\text { mean of autocorrelation }=\text { mean }(C(s, t))
$$

These features are computed for each of the obtained signal value (time and amplitude values) with 10 seconds windows, giving a total of features columns

$$
9(\text { values }) * 17 \text { (features per value })=153(\text { features columns })
$$

\subsubsection{Sympathetic and Parasympathetic Stimulations}

It is known that cognitive processes evoke patterned changes in physiological state of the body. This dynamic modulation of visceral state is neurally mediated by sympathetic and parasympathetic divisions of the autonomic nervous system [88]. The parasympathetic

nervous system (PSNS, or occasionally PNS) is one of the two main divisions of the autonomic nervous system (Figure). The autonomic nervous system is responsible for regulation of internal organs and glands, which occurs unconsciously. In a general sense, 
the ANS is considered to be an efferent system that controls visceral functions involving the heart, the gastro-intestines, and glandular secretion.

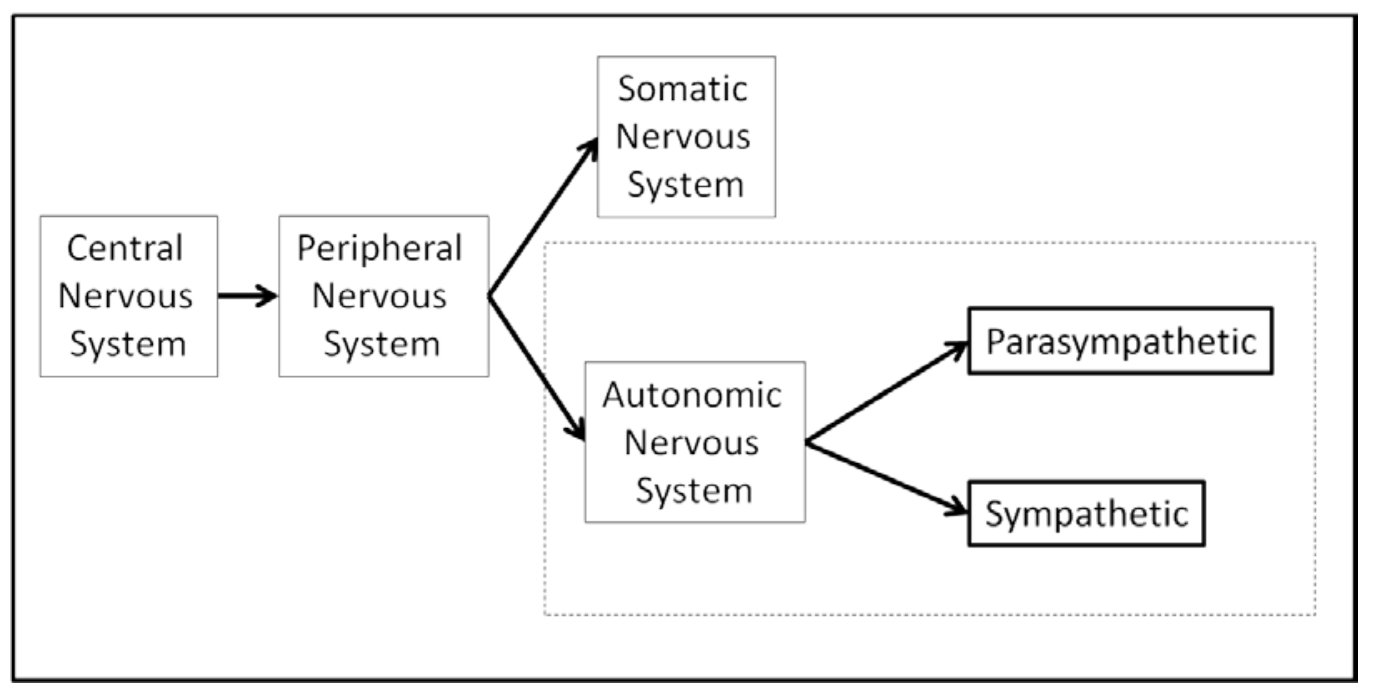

FIGURE 18: CENTRAL NERVOUS SYSTEM LAYOUT

The parasympathetic aspect of this system is responsible for stimulation of "rest-anddigest" activities that occur when the body is at rest. On the contrary the sympathetic nervous system is responsible for stimulating activities associated with the fight-or-flight response. Sympathetic and parasympathetic divisions typically function in opposition to each other. This natural opposition is more accepted as complementary in nature rather than antagonistic. In addition, studies of autonomic activity that accompanies attention, orienting and learning have demonstrated that the autonomic nervous system is not simply a 'non-cognitive' and automatic part of brain function. The autonomic and central nervous systems are intimately related, and new research is beginning to link brain function with autonomic function in a dynamic way [89]. 
Jennings and colleagues [90-92] have studied cognitive-autonomic interactions in a simple motor-preparation task, with a fixed foreperiod warning signal. The subject is presented with a light, which is followed 10 seconds later by a second light, and the subject presses a button as quickly as possible after the second light appears. Inhibition of the motor response during the warning period is accompanied by changes in autonomic action on the heart, such that the heart rate is decelerated. The motor inhibition may, moreover, be a component of the attention related processes that are activated in order for an action to be selected at the end of the foreperiod. However, it has been argued [93] that the minute changes in autonomic influences on the heart during the waiting period are induced by the cognitive processes that underlie 'expectancy'. With the directional motivation deduced from such studies, sympathetic and parasympathetic seemed a possible area of application that could enhance the categorization between the cases of attentiveness and the lack thereof in a subject.

Studies have assessed that the sympathetic nervous system stimulates heart's functioning and an increase in sympathetic stimulation reflects in an increase in hear rate. In contrast parasympathetic nervous system inhibits the hearts functions therefore an increase in parasympathetic stimulation causes a decrease in heart rate. So changes in heart rate directly translate to the fluctuations in Heart Rate Variability (HRV) indices. Hence HRV provides a valuable insight into the functioning of the autonomous nervous system. So taking the HRV values calculated using the ECG signal, a standard spectral analysis 
routine is applied upon it. Fourier Transform is applied on the HRV values which are computed from 10 second windows. Using Butterworth band-pass filtering method, the High Frequency (HF), Low Frequency (LF) components of the original signal are extracted. The HF power spectrum is evaluated in the range from 0.15 to $0.4 \mathrm{~Hz}$. This band reflects parasympathetic (vagal) tone and fluctuations caused by spontaneous respiration known as respiratory sinus arrhythmia. The LF power spectrum is evaluated in the range from 0.04 to $0.15 \mathrm{~Hz}$. This band can reflect both sympathetic and parasympathetic tone.

Using the information acquired in from the Fourier domain the following parameters are evaluated:

- Power of High Frequency (HF) 0.15 to $0.4 \mathrm{~Hz}$

- Power of Low Frequency (LF) 0.04 to $0.15 \mathrm{~Hz}$

- LF/HF Ratio

- The square root of the mean squared differences of successive RR intervals. (RMSSD)

The LF/HF Ratio is used to indicate balance between sympathetic and parasympathetic tone. A decrease in this score might indicate either increase in parasympathetic or decrease in sympathetic tone. It must be considered together with absolute values of both LF and HF to determine what factor contributes in autonomic misbalance. RMSSD Estimate of the short-term components of HRV thereby providing the Vagal Index. 
Therefore, the features that are computed form analysis of effects of autonomous nervous system are also added to the features obtained from signal value (time and amplitude values) with 10 seconds windows. Thereby giving a total of features columns 153 (HRV based features) +4 (ANS based features) $=157$ features in total.

These computed features are then sent to the machine learning stage for classification, training and testing. 


\subsection{Wavelet Transform}

As explained, sometimes analysis of a signal in time domain alone is insufficient, and transformation of the signal into different domains can help in better targeting the finer features in a signal. With time series signals an obvious domain transformation is into the frequency domain also known as the Fourier domain. Fourier transform helps in the analysis of the signals in the frequency domain; however in the Fourier domain time information is obscured since in this domain time information is averaged over the entire signal. So to overcome this shortcoming of Fourier transformation, Short Time Fourier Transform (STFT) is sometimes used. However STFT still averages time information over localized windows and hence does not truly represent both frequency and time information together. It tries to analyze finite length events in signals using sinusoidal basis functions that are not limited in time and it is also highly dependent on the size of the window.

Analysis methods which transform the time series data into a true representation of time-frequency domain allow the elucidation of local, transient or intermittent components of the original signal. There are several time-frequency analysis methods that are available for signal processing which basically perform high resolution decomposition in the timefrequency domain. STFT, Wigner-Ville transform (WVT), Choi-Williams distribution (CWD), Continuous Wavelet Transform (CWT), etc. are some of the available time-

frequency analysis methods. Of these the continuous wavelet transform has emerged as the most favored tool by researchers as it does not contain the cross terms inherent in the WVT 
and CWD methods while possessing frequency-dependent windowing which allows for arbitrarily high resolution of the high frequency signal components (unlike the STFT)[77] .

Many of the ideas behind wavelet transforms have been in existence for a long time. However, wavelet transforms analysis as we now know predominantly began in the mid-1980s. It was initially used to investigate seismic signals [94]. The application of wavelet transform analysis began taking more prominence at beginning of the 1990s within science and engineering. In recent years, Wavelet Transform analysis has proven consistently useful for bio-medical signal analysis. Some of the areas where Wavelet Transform has been used include: the EMG [95], EEG [96], clinical sounds [97], respiratory patterns [98], blood pressure trends [99] and DNA sequences [100].

It is known that cognitive processes evoke patterned changes in physiological state of the body. This dynamic modulation of visceral state is neurally mediated by sympathetic and parasympathetic divisions of the autonomic nervous system [88][1].

\subsubsection{Mathematical Formulation}

Wavelet transforms essentially exist in two distinct types: the Continuous Wavelet Transform (CWT) and the Discrete Wavelet Transform (DWT). For its practical implementation the continuous wavelet transform or CWT is computed over a finely discretized time-frequency grid. This discretization involves an approximation of the transform integral (i.e. a summation) computed on a discrete grid of a scales and b locations. 


$$
W_{\Psi, x}(a, b)=\frac{1}{\sqrt{|a|}} \int_{-\infty}^{+\infty} x(t) \Psi^{*}\left(\frac{t-b}{a}\right) d t \quad a \neq 0
$$

\section{Synthesis Equation (Inverse CWT)}

$$
x(t)=\frac{C_{\Psi}^{-1}}{a^{2}} \int_{-\infty-\infty}^{+\infty} \int_{-\infty}^{+\infty} W_{\Psi, x}(a, b) \Psi\left(\frac{t-b}{a}\right) d a d b \quad a \neq 0
$$

Where

- $\Psi^{*}(\mathrm{t})$ is the complex conjugate of the analyzing wavelet function $\Psi(\mathrm{t})$, or the mother wavelet.

- $\quad b$ is the shifting or dilation parameter of the wavelet and

- $a$ is the scaling or location parameter of the wavelet.

- $C_{\Psi}^{-1}$ is a constant

In general, the wavelet transform is approximated in this way over each time step for a range of wavelet scales; the inverse continuous wavelet transform is also computed as a discrete approximation. CWT in its true definition demands the use of all values of shifting and scaling. Therefore CWT is a heavy computational burden and in general an order or two in magnitudes more wavelet values generated than original signal components, thus 
leading to a lot of redundant information. In fact, the computation of ICWT requires double integration hence making the computation more expensive.

To overcome these issues with CWT, in this study for the analysis of the ECG signal the Discrete Wavelet Transform (DWT) method has been employed. The advantages of using DWT is that it allows the analysis of continuous signals by applying only discrete values of shift and scaling to form the discrete wavelets. Also, if the original signal is sampled with suitable set of scaling and shifting values, the entire continuous signal can be reconstructed from the DWT (using Inverse-DWT). A natural way of setting up the parameters $a$ (scaling) and $b$ (shifting) is to use a logarithmic discretization of the ' $a$ ' scale and link this respectively to the step size taken between ' $b$ ' locations or shifts. To link ' $b$ ' to ' $a$ ' discrete steps are taken to each location ' $b$ ', which are proportional to the ' $a$ ' scale. This kind of mother wavelet can be shown in the following form;

Discrete Mother Wavelet Representation

$$
\Psi_{m, n}(t)=\frac{1}{\sqrt{a_{0}^{m}}} \Psi\left(\frac{t-n b_{0} a_{0}^{m}}{a_{0}^{m}}\right)
$$

Where

- Integer's $m$ and $n$ control the wavelet shifting and scaling respectively

- $a_{0}$ is a specified fixed dilation step parameter set at a value greater than 1

- $b_{0}$ is the location parameter which must be greater than zero.

Analysis Equation (DWT) 


$$
W_{m n}=\int_{-\infty}^{+\infty} x(t) \Psi_{m n}^{*}(t) d t
$$

Synthesis Equation (Inverse DWT)

$$
\mathrm{x}(\mathrm{t})=\mathrm{c} \sum_{m n} \mathrm{~W}_{m n} \Psi{ }_{m n}(\mathrm{t})
$$

(Where c is a constant associated with the mother wavelet)

The application of DWT on the acquired and preprocessed ECG signal is explained next.

\subsubsection{Application of Discrete Wavelet Transform}

In this study, Discrete Wavelet Transform or DWT is applied to the pre-processed ECG signal.

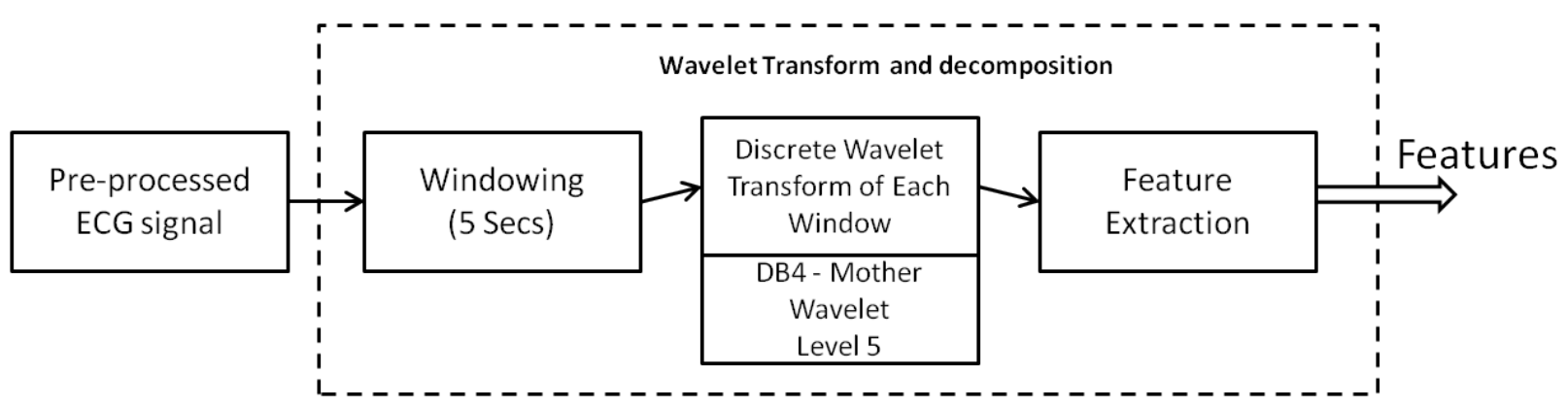

FIGURE 19. DISCRETE WAVELET TRANSFORM APPLICATION STEPS 
Windowing: As shown in the figure18, the raw ECG signal after preprocessing is first sent through the windowing step. In this step the interesting and boring portions of the ECG which are extracted during the pre-processing stage are sent through a windowing mechanism. In this mechanism, the pre-processed ECG signal is partitioned into tiny windows. These windows are non-overlapping and contain ECG data of 10 sec interval $(128 \mathrm{~Hz} * 10 \mathrm{sec}=640$ data-points $)$.

During experimentation, various window sizes were tried and tested. The window size of 10 seconds seemed to be the smallest window size to provide an ideal quality of features and result. With the vision of a practical application of this research, a system such as this would provide live results as the data is being collected at real-time. The system would use the last 10 seconds of acquired data to predict its classification output. A larger window size of $10+$ seconds for example would allow the system to average the output predictor based on an average feature extraction over a larger time period, which in turn might reduce the precision and accuracy of such a system.

Discrete Wavelet Transform: Each of the 10 second partitioned window is decomposed separately using DWT. A key aspect of DWT application is the choice of the mother wavelet which is used for the decomposition. Choosing an optimal wavelet function is a mootable issue, which depends on the application and the signals characteristics [101]. A common heuristic is to choose a mother wavelet which is as similar as possible in shape to the signal of interest [102]. Therefore in this experiment, a wavelet needed to be chosen which is similar in shape to the ECG sinus rhythm, with peaks resembling each of the 
significant point on the P-QRS-T complex. Amongst the many available families of wavelets available, some of the more popular ones for ECG decomposition are Daubechies proposed functions (db2, db3, db4, db5, db6, db7, db8) [103]. Symlets wavelets (sym2, sym3, sym4, sym5, sym6, sym7, sym8) within which sym3 and syn8 are commonly used [104]. There is also the family of biorthogonal wavelets (bior3.3, bio4.4, bio6.8) which are sometimes used for ECG applications [105].

Ingrid Daubechies, one of the pioneers on wavelet research, invented what are called compactly supported orthonormal wavelets -- thus making discrete wavelet analysis practicable. The names of the Daubechies family wavelets are written $\mathrm{dbN}$, where $\mathrm{N}$ is the order, and db the "surname" of the wavelet. After much experimentation, 'db4' from this family of Daubechies wavelets was chosen as the wavelet of choice for this study since it seemed to best fit the composite nature of the ECG signal acquired from the armband. 


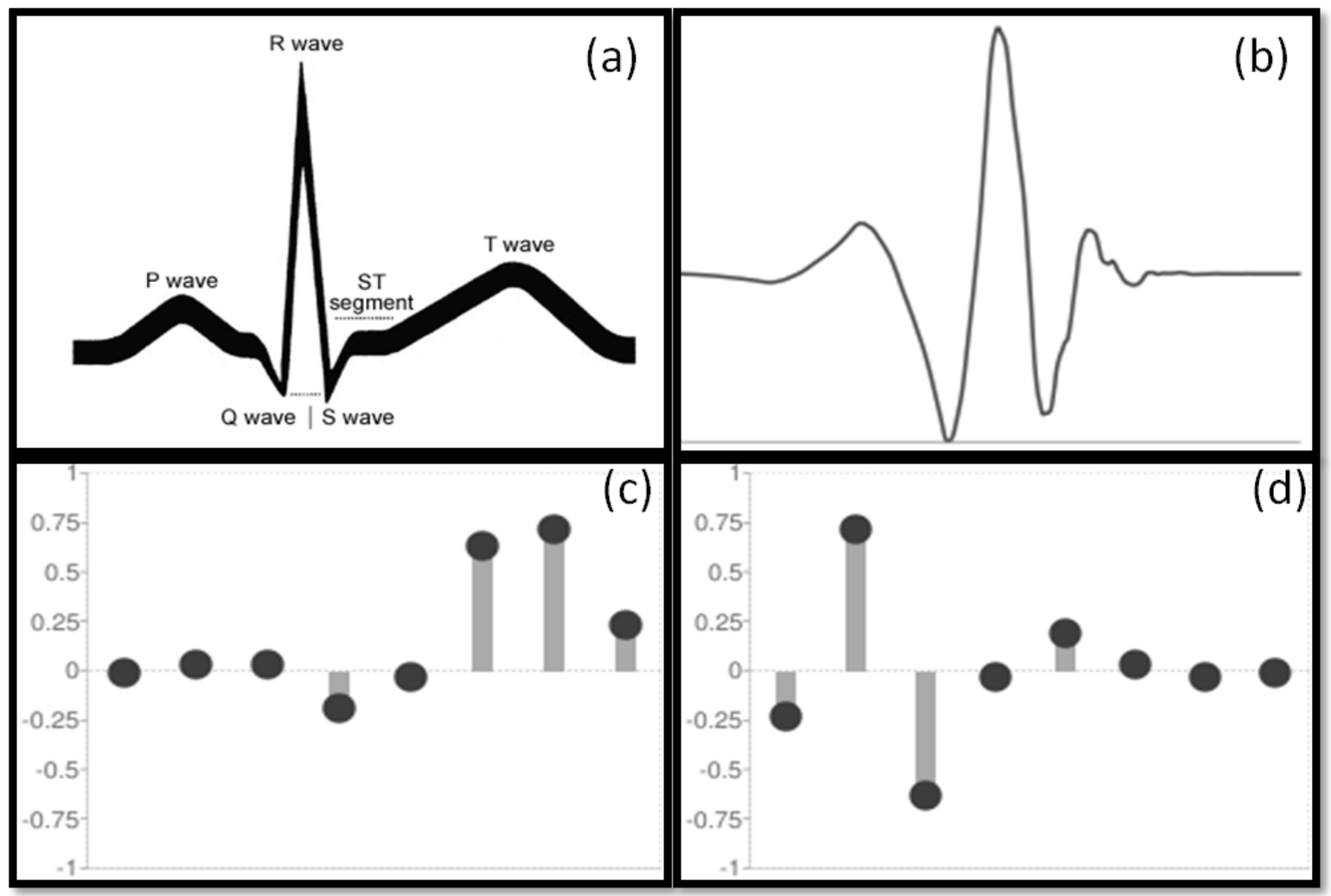

FIGURE 20. (A) IS THE SAMPLE ECG WAVE, (B) IS THE DB4 WAVELET FUNCTION, (C) DECOMPOSITION LOWPASS FILTER FOR DB4, AND (D) DECOMPOSITION HIGH-PASS FILTER FOR DB4.

In the Wavelet Transform procedure, using the chosen mother wavelet (in this case being db4), the pre-processed ECG signal is decomposed into cascading levels of filter banks by using low and high pass filter. The figure20 below shows the procedure in more detail; 


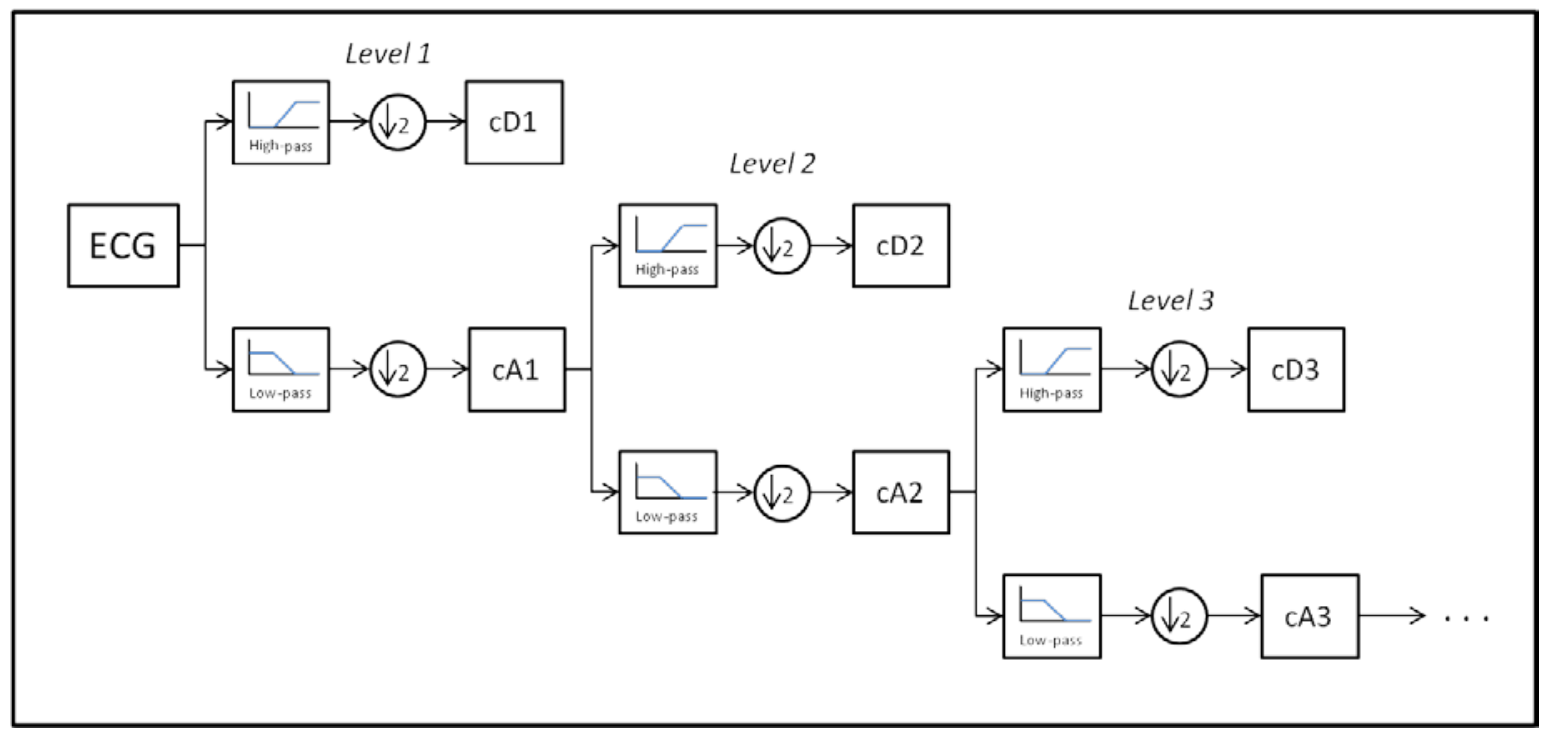

FIGURE 21. DECOMPOSITION PROCESS OF WAVELET TRANSFORM

The decomposition process in wavelet transform can be performed iteratively into several levels. The number of levels chosen for decomposition is application specific and also depends on the complexity of the signal. For the ECG signal used in this study, 5 levels of decomposition seemed to provide all the required useful information; further decomposition did not yield a better result.

As shown in the figure, the wavelet transform mechanism works with successive approximation with varying scale and shift levels of the mother wavelet so that the original signal is broken down into many lower resolution components. A computational viewpoint of the decomposition can be visualized as the signal passing through a low and high pass filter at each level. The output of the high pass filter yields approximation coefficients and the output of the low pass filter yields the detailed coefficients of the signal. Here cAn stands for approximation coefficient at level ' $n$ ' similarly; cDn stands for detail coefficient at level ' $n$ '. During each filter operation the signal is down sampled to roughly half the size 
input signal. The decomposition of each successive level is performed on the approximation coefficients of the previous level.

\subsubsection{Feature Extraction}

As mentioned previously, wavelet transform is performed on each of the 10 seconds windows that are extracted from the pre-processed ECG signal. The result after the decomposition of each window is the array of approximation and detail coefficients which comprise of the different resolution components of the signal. Since 5 levels of decomposition is performed for each window, feature extraction is performed on the detailed coefficient of each level and approximation coefficient of the final level. This ensures that each decomposed resolution of the original signal is utilized for the feature extraction step.

The features computed from these coefficients are as follows: (Here, $\left(\mathrm{x}_{1}, \mathrm{x}_{2}, \ldots \mathrm{x}_{\mathrm{n}}\right)$ represents the values of each coefficient from the $10 \mathrm{sec}$ window)

- Standard Deviation

Given in equation (8)

- Median

$$
\text { median }=\text { middle_value }\left(\operatorname{sort}_{\text {ascending }}\left(x_{1}, x_{2}, \ldots, x_{n}\right)\right)
$$

- Minimum

Given in equation (6)

\section{- Maximum}


Given in equation (7)

- Window Mean

Given in equation (9)

- Energy

Given in equation (10)

- Power

Given in equation (11)

- Slope

Given in equation (12)

\section{- Entropy}

Entropy is a statistical measure of randomness. It is very useful in evaluating the information present within a signal.

$$
\text { entropy }=-\operatorname{sum}(p \cdot * \log 2(p))
$$

(where $\boldsymbol{p}$ is the histogram of the signal)

\section{- Skweness}

Skewness is a measure of the asymmetry of the data around the sample mean. If skewness is negative, the data are spread out more to the left of the mean than to the right. If skewness is positive, the data are spread out more to the right. The skewness of the normal distribution (or any perfectly symmetric distribution) is zero. 
Equation provided in (13)

- Kurtosis

Kurtosis is a measure of how outlier-prone a distribution is. The kurtosis of the normal distribution is 3. Distributions that are more outlier-prone than the normal distribution have kurtosis greater than 3; distributions that are less outlier-prone have kurtosis less than 3 .

Equation provided in (14)

- Range

Equation provided in (15)

- Signal Complexity

Equation provided in (19)

- Signal Mobility

Equation provided in (20)

- Log of Variance

Equations provided in $(21,22,23)$

- Mean of frequencies (Fourier domain)

Equation provided in $(24,25)$

- Variance of probability distribution 
Equation provided in $(27,28)$

\section{- Sum of Auto-Correlation}

Equation provided in $(29,30)$

- Mean of Auto-Covariance

Equation provided in $(21,32)$

- Entropy of Frequency

This feature is computed using all level of detailed and final level of approximation coefficient. Hence this feature is added separately to each window.

$$
\text { Entorpy }(\text { frequencies })=-p \log (p)
$$

And $p$ is calculated as follows:

$$
p=\frac{\sum_{j=1}^{n}\left|A p p r_{j}\right|^{2}}{n \sum_{l=1}^{5} \sum_{i=1}^{m} \operatorname{Detl}_{i}}
$$

where $a c j$ are the approximation coefficients at level $5, \mathrm{n}$ is the number of elements in the approximate coefficient window and $d c i$ are the detail coefficients at level $l(1-5)$, $\mathrm{m}$ is number of elements in the detailed coefficient at each level. 
After the feature extraction has been performed, the total feature set for the wavelet transform step will contain;

(6 coefficients $(5$ detailed +1 approximation $) * 19$ (features per coefficient) $)+1$ (feature computed with all levels) $=115$ (features columns)

These computed features are then sent to the machine learning stage for classification, training and testing. 


\subsection{S-Transform (Stockwell transform)}

The S transform was proposed by R.G. Stockwell and his coworkers 1996. The distinction of $\mathrm{S}$ transform is that it produces decomposition of frequency dependant resolution in the time-frequency domain while entirely retaining the local phase information. In other words, The S-transform not only estimates the local power spectrum, but also the local phase spectrum, which is highly desirable in studying complex physiological signals such as the ECG.

When it comes to analyzing dynamic spectrum or local spectral nature of nonstationary observations such as the ECG some of the popular methods include, Short Time Fourier Transform (STFT) [106], Gabor transform [107], complex demodulation [108] which produces a series of band pass filtered voices and is also related to the filter bank theory of wavelets etc. Some methods represent the transformation in a combination of time and frequency domain such as the Cohen class [109] of generalized time-frequency distributions (GTFD), Cone-Kernel distribution[110], Choi-Williams distribution [111] as well as the smoothed pseudo Wigner distribution (PWD) [112]. However, one of the more popular methods for decomposition and analysis in time-frequency domain is Wavelet Transform, application of which in this study has already been described in detail.

Discrete Wavelet Transform or DWT performs decomposition of a signal that provides excellent time resolution while maintaining key spectral information or frequency resolution. However, the details of frequency responses can sometimes be sparse and variation in the dilation can lead to overlapping spectral response. Hence for identifying 
the more subtle and finer engrained changes in the ECG characteristics between 'interesting' and 'non-interesting' portions of the data acquisition routine, a clearer timefrequency representation of the ECG is desired.

Although $\mathrm{S}$ transform is similar to continuous wavelet transform in having progressive resolution, unlike wavelet transform, the S-transform retains absolutely referenced phase information. Absolutely referenced phase implies that the phase information calculated by the S-transform is referenced to time $t=0$, which is also true for the phase given by the Fourier transform. Only difference being the S transform provides the absolute referenced phase information for each sample of the time-frequency space.

\subsubsection{Mathematical Formulation}

There are two varieties of S-transform, continuous and discrete. The continuous S transform [113] is essentially an extension of the STFT. It can also be seen as a phase corrected format of the Continuous Wavelet Transform (CWT).

The STFT of a signal $h(t)$ is defined as

$$
\operatorname{STFT}(\tau, f)=\int_{-\infty}^{\infty} h(t) g(\tau-t) e^{-j 2 \pi f t} d t
$$

Where

- $\tau$ is the time of spectral localization,

- $f$ is the Fourier frequency

- and $g(t)$ denotes a window function. 
The $\mathrm{S}$ transform can be derived from the above STFT equation simply by substituting the window function $g(t)$ the Gaussian function,

$$
g(t)=\frac{|f|}{\sqrt{2 \pi}} e^{-\frac{t^{2} f^{2}}{2}}
$$

Therefore the S transform be mathematically defined as follows;

$$
S(\tau, f)=\int_{-\infty}^{\infty} h(t) \frac{|f|}{\sqrt{2 \pi}} e^{-\frac{(\tau-t)^{2} f^{2}}{2}} e^{-j 2 \pi f t} d t
$$

Since S transform essentially functions with the Gaussian window during decomposition, it can be deduced that with a wider window in the time domain the transformation can provide better resolution for lower frequency, and with a narrow Gaussian window the resolution for higher frequency is better accentuated.

For application of $\mathrm{S}$ transform in this study, the continuous $\mathrm{S}$ transform does not prove to be a practice choice. Simply because the acquisition of the ECG signal itself was performed with discrete sampling and also a continuous decomposition of this signal for all frequencies can time is simply not computationally pragmatic. Hence a Discrete version of the S transform has been adopted for the decomposition of the ECG signal.

The Discrete S Transform can be presented as follows;

Let $h[k T]$ be the discrete time series signal to be investigated, where $k=0,1, \ldots, N-1$ and $T$ is the time sampling interval. The discrete format of the Fourier transform can be shown as follows; 


$$
H\left[\frac{n}{N T}\right]=\frac{1}{N} \sum_{k=0}^{N-1} h[k T] e^{\frac{2 j \pi n k}{N}}
$$

Using the continuous S transform equation and the above equation, the time series, $h[k T]$ 's

S transform can be represented as follows; (making $f \rightarrow n / N T$ and $\tau \rightarrow j T$ )

$$
S\left[j T, \frac{n}{N T}\right]=\sum_{m=0}^{N-1} H\left[\frac{m+n}{N T}\right] e^{\frac{2 \pi^{2} m^{2}}{n^{2}}} e^{\frac{2 j \pi m j}{N}} \quad n \neq 0
$$

Where $\mathrm{j}, \mathrm{m}$, and $n=0,1, \ldots, \mathrm{N}-1$.

\subsubsection{Application of S Transform}

In this study, Discrete S Transform is applied to the pre-processed ECG signal.

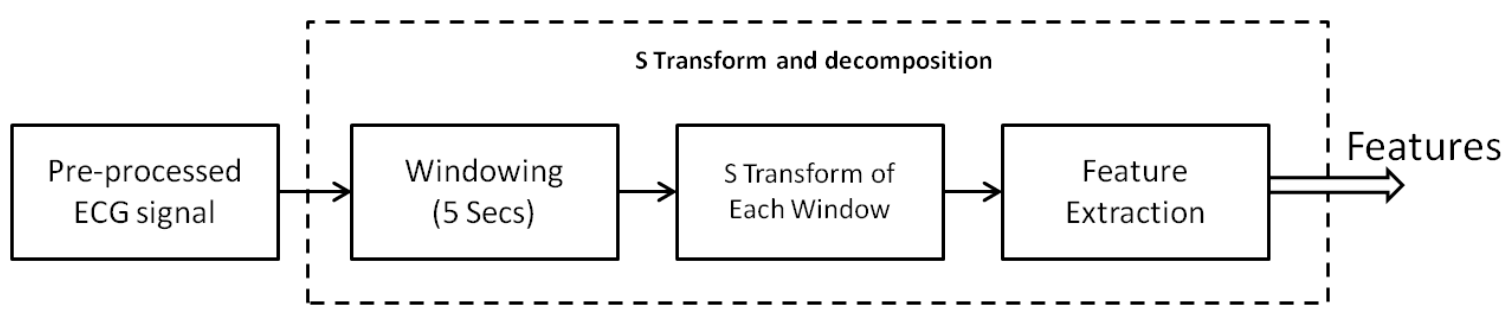

FIGURE 22. S TRANSFORM APPLICAION STEPS

Windowing: Similar to the windowing method in the Wavelet Transform step, the raw ECG signal after preprocessing is first sent through the windowing step. In this step the interesting and boring portions of the ECG which are extracted during the pre-processing stage are sent through a windowing mechanism. In this mechanism, the pre-processed 
ECG signal is partitioned into tiny windows. These windows are non-overlapping and contain ECG data of $10 \mathrm{sec}$ interval (128 Hz * 1- sec = 1280data-points).

For factors of consistency and for practical applications reasons, the window size is chosen as 10 seconds here as well.

$S$ transform of each window: After the windowing step, each of the 10 seconds long windows are defragmented using S-transform. The output of the S transform is a complex 2 dimensional matrix with rows representing the frequencies and the columns represent the time values. The $\mathrm{S}$ transform algorithm applied in this study is tuned to produce a step wise frequency range with step size being $1 \mathrm{~Hz}$ and the time interval between samples in the result is 1 step unit.

An example output of a 5 second window of an ECG data after S-transform is given as follows (for example purposes and details in viewing the image only a 5 second window has been illustrated); 


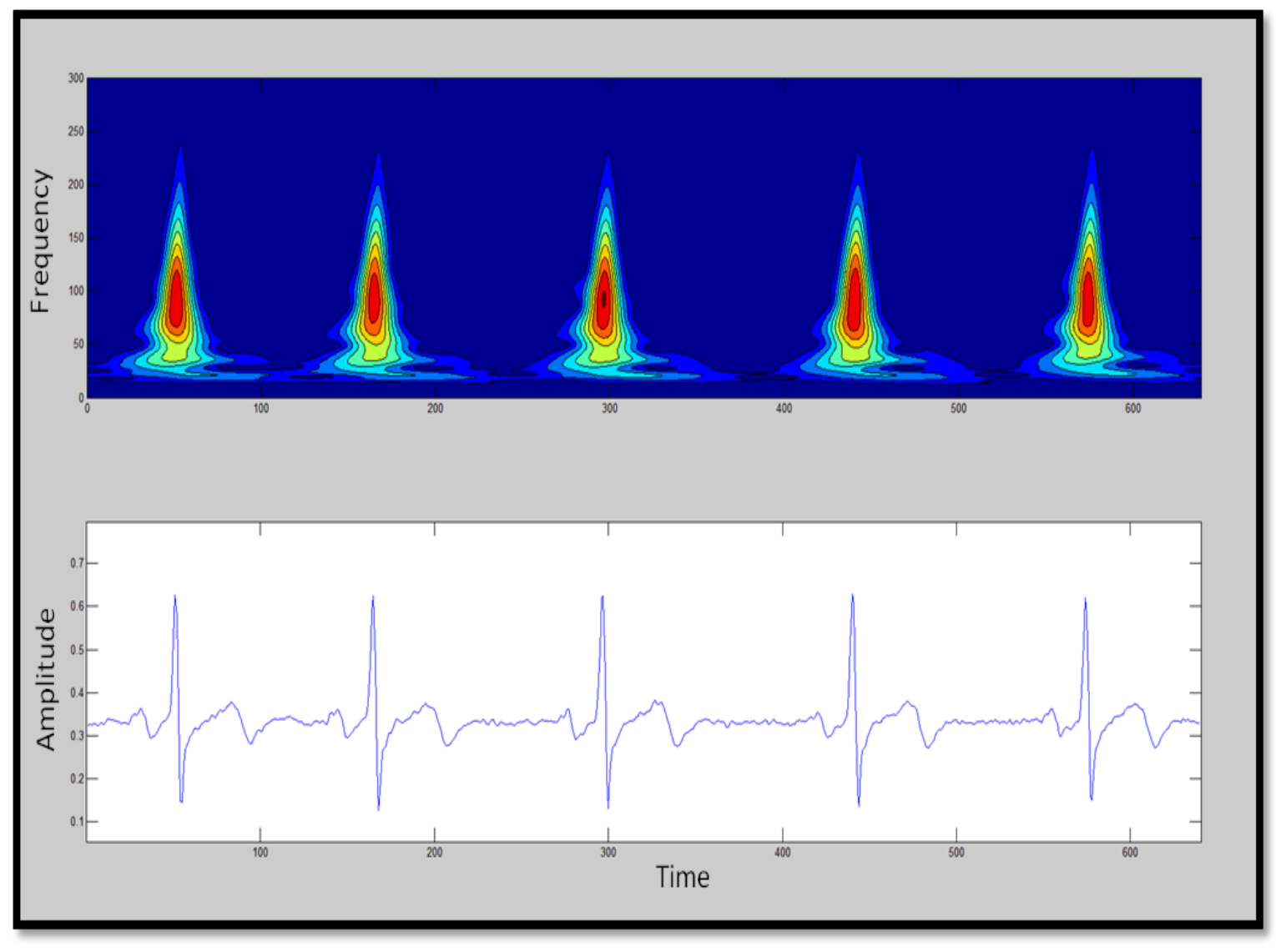

FIGURE 23. CONTOUR BASED VISUALIZATION OF AN ST WINDOW

The above figure22 shows the exact point to point representation of the original signal in the $\mathrm{S}$ transforms time-frequency domain. The $\mathrm{S}$ transform output matrix has been shown in a contour map display.

\subsubsection{Feature Extraction}

The output of each window is a frequency-time represented matrix. Each instance of the matrix is frequency point and a time point (by the row and column position respectively). So the entire output matrix can be presented as follows $S T(x, y)$, where $x$ is the frequency (row) location, and $y$ is the time (column) location. 
The extraction of features form the derived output matrix of ST is performed in two steps. First the output matrix is reduced from two dimensions to a single dimension. This is done by computing certain common statistics along the frequency dimension $x$, while retaining the discreteness in the time dimension $y$ as is.

The computed features along frequency for each time step $y$ are:

- Mean of frequencies $(f)$

$$
\operatorname{mean}(f)=\frac{\sum_{i=1}^{x} S T_{i, y}}{n}
$$

- Sum of frequencies $(f)$

$$
\operatorname{sum}(f)=\sum_{i=1}^{x} S T_{i, y}
$$

- Product of frequencies $(f)$

$$
\operatorname{product}(f)=\left(S T_{1, y} * S T_{2, y} * S T_{3, y} * \ldots * S T_{x, y}\right)
$$

- Standard Deviation of frequencies $(f)$

$$
\operatorname{std}(f)=\frac{1}{x} * \sum_{i=1}^{x} S T_{i, y}^{2}
$$

- Range

$$
\begin{aligned}
& \text { range }=\operatorname{maximum}\left(S T_{1, y}, S T_{2, y}, \ldots, S T_{x, y}\right) \\
& -\operatorname{minimum}\left(S T_{1, y}, S T_{2, y}, \ldots, S T_{x, y}\right)(50)
\end{aligned}
$$

At the end of step one we get an array of features from the frequency domain as follow:

$$
\text { Freq }_{f e t s}=[\text { mean }(f), \operatorname{sum}(f), \operatorname{product}(f), \operatorname{std}(f), \operatorname{range}(f)]
$$


The next step is to compute statistical features along the time domain:

- Mean

$$
\operatorname{mean}(S T)=\operatorname{mean}\left(f_{i}\right) ; \quad \text { where } f_{i} \in \text { Freq } q_{\text {fets }}
$$

- Sum

$$
\operatorname{sum}(S T)=\operatorname{sum}\left(f_{i}\right) ; \quad \text { where } f_{i} \in \text { Freq }_{\text {fets }}
$$

- Mean of Auto-Covariance

$$
\text { mean }(\text { autocovariance }(S T))=\text { mean }\left(\operatorname{autocovariance}\left(f_{i}\right)\right) \text {; }
$$

where $f_{i} \in$ Freq $q_{\text {fets }}$

- Sum of Cross-Correlation

$$
\begin{aligned}
& \operatorname{sum}(\text { autocorrilation }(S T))=\operatorname{sum}\left(\text { autocorrilation }\left(f_{i}\right)\right) ; \\
& \text { where } f_{i} \in \text { Freq }_{\text {fets }}
\end{aligned}
$$

- $\log _{2}$ of Variance

$$
\begin{aligned}
& \log _{2}(\text { variance }(S T))=\log _{2}\left(\text { variance }\left(f_{i}\right)\right) ; \\
& \text { where } f_{i} \in \text { Freqfets }
\end{aligned}
$$

Two other features that are calculated are:

- Mean of max frequencies

$$
\operatorname{mean}(\max (S T))=\operatorname{mean}\left(\max \left(S T_{1, y}, S T_{2, y}, \ldots, S T_{x, y}\right)\right)
$$

- Mean absolute deviation of frequencies

$$
\operatorname{mean}(\operatorname{abs}(S T))=\operatorname{mean}(\operatorname{abs}(S T-\operatorname{mean}(S T)))
$$


After the feature extraction has been performed, the total feature set for the S-Transform step will contain;

$(5($ features in step 1$) * 5($ features in step 2$))+2($ additional non iterative features $)=27$ (features columns per window) 


\subsection{Dual Tree Complex Wavelet Transform -An Introduction.}

The Discrete Wavelet Transform (DWT) is most commonly used in its maximally decimated format also known as dyadic filter tree form as shown in the figure in Wavelet Transform section. Over the years DWT has been utilized with great success across a variety of signal processing applications $[114,115]$. In a nutshell, the DWT replaces the infinitely oscillating sinusoidal basis functions of the Fourier transform with a set of locally oscillating basis functions called wavelets.

Despite DWT's enormous success in various applications, and despite its computational efficiency and sparse representation, the Wavelet transform suffers from a few fundamental and interrelated disadvantages.

- Oscillations

Developing singularity extraction and modeling the signal during wavelet based processing gets considerably complicated [116] since wavelets are essentially bandpass functions, which results in the oscillation of the wavelet coefficients around the positive and negative singularities.

- Shift Variance

In the wavelet domain processing, shift variance also complicates the processing, since a tiny shift of the signal can cause a disturbance in the oscillation patterns of the wavelet coefficient around singularities [117, 117, 118].

- Aliasing

Wavelet Coefficients are computed using discrete time down sampling functions which are bestrewed with non-ideal low and high pass filters, thereby resulting in 
substantial aliasing. Hence after decomposition of a given signal, if the resulting coefficients are processed in any manner, then the inverse or the synthesis function will reconstruct the signal with artifacts.

- Lack of Directionality The result of wavelet transform produces a pattern that is oriented along multiple directions. This lack of direction influences the processing of multi-dimensional signal analysis such as application of DWT on image, such as edge detection [119].

The Dual-Tree Complex Wavelet Transform (DTCWT) claims to overcome all the above shortcomings of a traditional DWT.

DCTWT Framework: The DCTWT was first introduced by Kingsbury in 1998 [117, 120]. The dual-tree approach is quite similar to standard DWT where in positive and negative bandpass filtering of the signals is performed at each level of decomposition. However, in DCTWT, two real DWT's are used simultaneously at each decomposition level. The first DWT gives the real part of the transform while the second DWT gives the imaginary part. The filter banks of analysis and reconstruction or synthesis of the DCTWT have been illustrated in the figures below. 


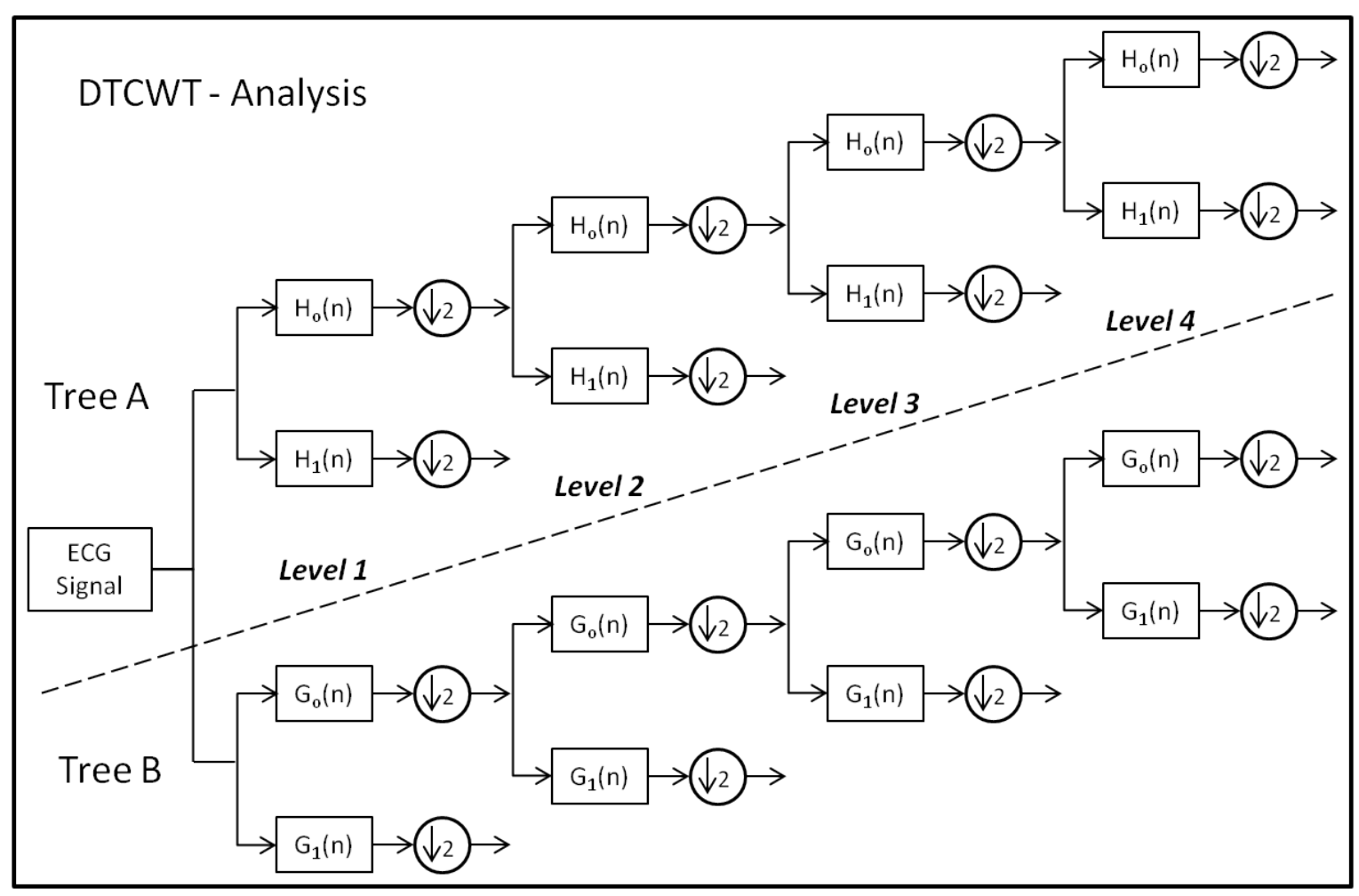

FIGURE 24. DUAL TREE WAVELET ANALYSIS ILLUSTRATION

Here $H_{0}(n)$ and $H_{1}(n)$ high pass and low pass filters respectively for tree $A$. Similarly, $G_{0}(n)$ and $G_{1}(n)$ are high and low pass filters for tree B. At each level of decomposition the input signal is down sampled to roughly half its original size. Also the output of each level is the detailed and approximation coefficient of the input signal. 


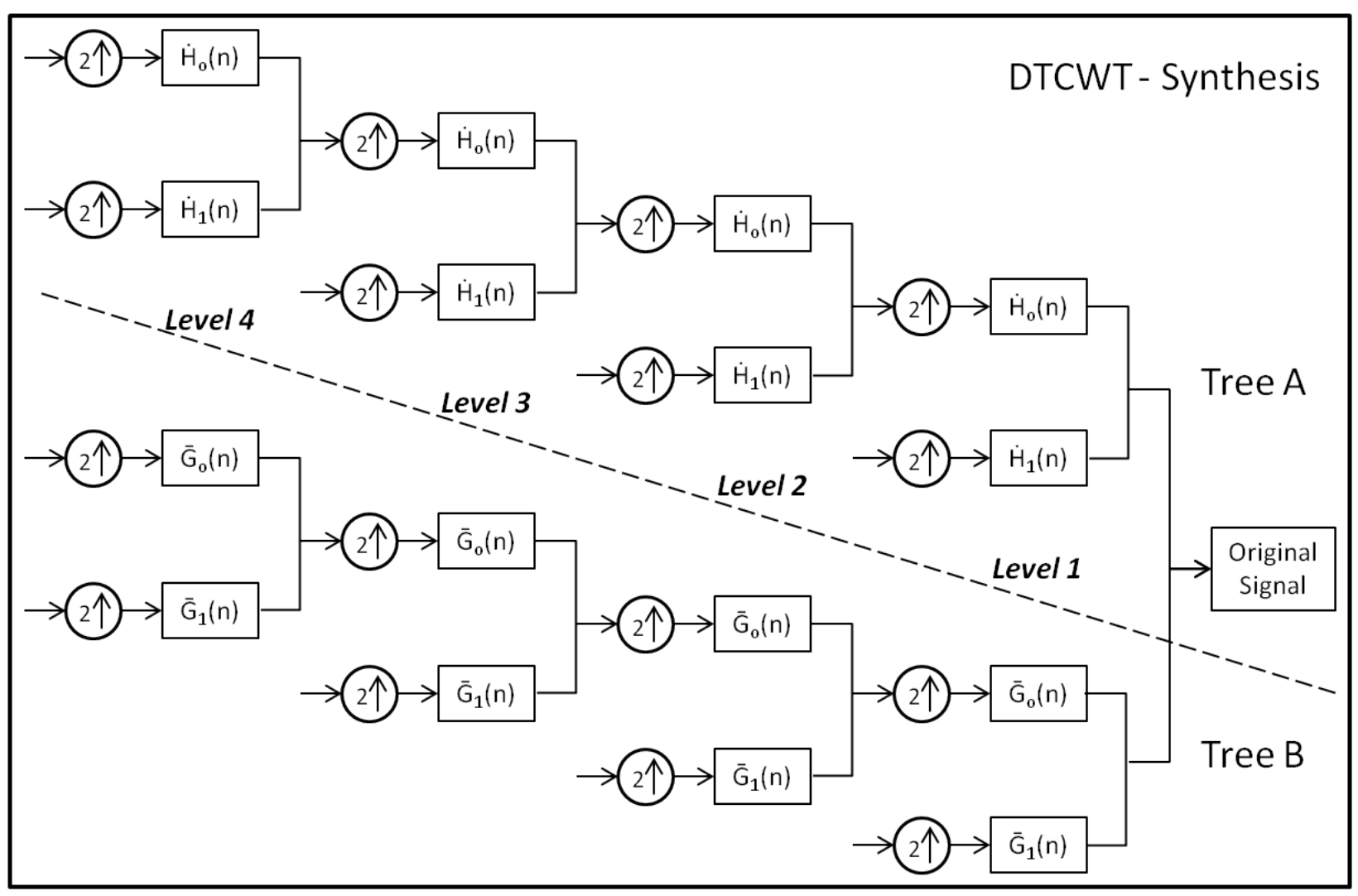

FIGURE 25. DUAL TREE WAVELET SYNTHESIS ILLUSTRATION.

Here $\dot{\mathrm{H}}_{0}(\mathrm{n})$ and $\dot{\mathrm{H}}_{1}(\mathrm{n})$ high pass and low pass filters respectively for tree A. Similarly, $\overline{\mathrm{G}}_{0}(\mathrm{n})$ and $\overline{\mathrm{G}}_{1}(\mathrm{n})$ are high and low pass filters for tree B. At each level of decomposition the input signal is down sampled to roughly half its original size.

It is important to understand that the Fourier transform does not suffer from the four problems listed for DWT. An essential aspect of this is that the magnitude of the Fourier transform does not oscillate positive and negative but rather provides a smooth positive envelope in the Fourier domain. Furthermore, the magnitude of Fourier transform has a simple linear phase offset which encodes the shift thereby making it shift invariant. Also the coefficients of Fourier are not aliased and do not rely on a complicated aliasing 
cancelation property to reconstruct the signal. Finally, the sinusoids of the M-D Fourier basis are highly directional plane waves.

The reason for Fourier transform not suffering from such problems is simply because it is based on complex-valued oscillating sinusoids.

$$
e^{j \Omega t}=\cos (\Omega t)+j \sin (\Omega t)
$$

With $\mathrm{j}=\sqrt{-1}$. The oscillating cosine and sine components that form the real and imaginary parts respectively form a Hilbert transform pair. Thereby, they are $90^{\circ}$ out of phase with each other. Together they form an analytical signal $e^{j \Omega t}$. With the same ideology developing a Complex Wavelet Transform (CWT) as provided in equations 35 and 36 but with a complex valued scaling function along with a complex valued wavelet.

$$
\psi_{c}(t)=\psi_{r}(t)+j \psi_{i}(t)
$$

Here $\psi_{r}(t)$ is real and even while $j \psi_{i}(t)$ is imaginary and odd. Just as in the case of the complex sinusoidal in Fourier transform the $\psi_{r}(t)$ and $j \psi_{i}(t)$ form an Hilbert transform pair with $90^{\circ}$ phase shifts.

Projecting the signal on to $2^{\frac{j}{2}} \psi_{c}\left(2^{j} t-n\right)$, we get the complex format of the wavelet coefficient

$$
d c(j, n)=d_{r}(j, n)+j d_{i}(j, n)
$$

With the magnitude as $\left|d_{c(j, n)}\right|=\sqrt{\left[d_{r}(j, n)\right]^{2}+\left[d_{i}(j, n)\right]^{2}}$ 


$$
\begin{array}{r}
\text { And phase } \\
/ d_{c}(j, n)=\arctan \left(\frac{d_{i}(j, n)}{d_{r}(j, n)}\right) \\
\text { Where }\left|d_{c}(j, n)\right|>0
\end{array}
$$

Any CWT based on wavelets of compact support cannot exactly possess the Hilbert transform/analytic signal properties, and this means that any such CWT will not perfectly overcome the four DWT shortcomings. The key challenge in dual-tree wavelet design is thus the joint design of its two frequency bands to yield a complex wavelet and scaling function that are as close as possible to analytic. However the cost for all of this is a moderate redundancy produced by the two coefficients generated at each level for each filter, thereby Dual Tree Wavelet Transform falls under the expansive wavelet transform methods.

In application filter banks (FB) are used to perform analytic CWT splits at each output level. These filter banks are similar representatives of the mother wavelet in DWT however the filter banks are usually designed based on certain prerequisite conditions that allow the real and complex aspects of the low and high pass filters to be analytical towards complex wavelet applications. In this application the two filter banks used are 'qshift_b' which are Q-Shift 14,14 tap filters where Q shift represents $1 / 4$ samples shift at each level. The second type of filter bank used is 'near_sym_b' which is Near-Symmetric (closely related to the waveform of ECG) with 13, 19 tap filters. 


\subsubsection{Feature Extraction}

For feature extraction we use the real and imaginary coefficients derived from the DTCWT decomposition, and the real part of the approximate coefficient. Hence for each 10 sec window of the ECG signal, 5 levels of DTCWT is performed on it and the real and complex detailed coefficients from levels 1 through 5 for each level are taken and the real values of the $5^{\text {th }}$ level approximate coefficient is considered.

The features computed from these coefficients are as follows: (Here, $\left(\mathrm{x}_{1}, \mathrm{x}_{2}, \ldots \mathrm{x}_{\mathrm{n}}\right)$ represents the values of each coefficient from the $10 \mathrm{sec}$ window)

- Standard Deviation

Given in equation (8)

- Median

$$
\text { median }=\text { middle_value }\left(\operatorname{sort}_{\text {ascending }}\left(x_{1}, x_{2}, \ldots, x_{n}\right)\right)
$$

- Minimum

Given in equation (6)

- Maximum

Given in equation (7)

- Window Mean

Given in equation (9)

- Energy 
Given in equation (10)

- Power

Given in equation (11)

- Slope

Given in equation (12)

- Entropy

Given in equation (39)

\section{- Skweness}

Skewness is a measure of the asymmetry of the data around the sample mean. If skewness is negative, the data are spread out more to the left of the mean than to the right. If skewness is positive, the data are spread out more to the right. The skewness of the normal distribution (or any perfectly symmetric distribution) is zero.

Equation provided in (13)

\section{- Kurtosis}

Kurtosis is a measure of how outlier-prone a distribution is. The kurtosis of the normal distribution is 3. Distributions that are more outlier-prone than the normal distribution have kurtosis greater than 3; distributions that are less outlier-prone have kurtosis less than 3.

Equation provided in (14) 
- Range

Equation provided in (15)

- Signal Complexity

Equation provided in (19)

- Signal Mobility

Equation provided in (20)

- Log of Variance

Equations provided in $(21,22,23)$

- Mean of frequencies (Fourier domain)

Equation provided in $(24,25)$

- Variance of probability distribution

Equation provided in $(27,28)$

- Sum of Auto-Correlation

Equation provided in $(29,30)$

- Mean of Auto-Covariance

Equation provided in $(21,32)$ 
- Entropy of Frequency

Equation provided in $(40,41)$

After the feature extraction has been performed, the total feature set for the wavelet transform step will contain;

(11 coefficients (10 detailed +1 approximation) $* 19$ (features per coefficient) $)+1$

(feature computed with all levels) = 210 (features columns)

These computed features are then sent to the machine learning stage for classification, training and testing. 


\section{CHAPTER 6 Analysis of Electroencephalogram}

\subsection{Overview}

Electroencephalograms or EEG is the physiological signal that maps the activity of the neurons in the brain by measuring electrical potentials through surface electrodes which are strategically placed on the scalp of an individual. The EEG signal exhibits complex behavior and non-linear dynamics. In the past wide range of work has been done in understanding the complexities associated with the brain through multiple windows of mathematics, physics, engineering and chemistry, physiology etc [121, 122].

The EEG was first recorded by German psychiatrist, Hans Berger in 1929. Since then it has become an irreplaceable aspect of medical and clinical diagnostics. EEG is heavily used in diagnosis and management of patients with seizure disorders, brain tumors, stroke, brain damage cause through trauma, sleep disorders, and several other neurological conditions characterized by abnormal and distinctive wave patterns. A variety of remarkable diagnostic techniques have been developed over the last few decades, since EEG provides a convenient and relatively inexpensive way to study and understand the physiological manifestations of the brain.

In relation to this research, the use of EEG for neurofeedback (or biofeedback) is becoming increasingly popular. The use of EEG for detecting and identifying attention/focus in individuals is not a new concept. In 1997, Neuropathways EEG Imaging 
system became the first U.S. patented digital EEG neurofeedback device, displaying brain wave voltages and frequencies in real time, this was extensively used in the development of an interventional feedback system for helping children cope with Attention Deficit Hyperactive Disorder (ADHD) [123, 124]. Similar systems have also been developed for improving concentration and other cognitive functions of both Attention related disorder and head trauma patients [125].

The intention of acquiring and analyzing EEG in this project is to set a benchmark of sorts for attention recognition. The key point of this study is to see if physiological signals that can be collected from a portable armband can be comparably efficient in recognizing an individual's attention and focus. So the following section describes the signal processing methods used on the subjects EEG signals.

\subsection{Analysis Method}

EEG recordings are typically analyzed in an event-related fashion when one wants to gain insights into the relation of the EEG and experimental events. The standard analysis method is to focus on event-related potentials (ERPs) by averaging. ERPs are basically the measure of the brain response that is directly related to an internal of external stimulus. However, another approach is to concentrate on event related oscillations (EROs). ERO is generally a measure of brain response to a typical activity over a period of time. Oscillatory activity in the brain is widely observed at different levels of observation and is thought to play a key role in many cognitive functions such as information transfer, perception, motor control and memory [126-128]. 
Since in this study detecting and identifying a particular type of response or ERP based on a single activity is not the objective, the focus is more towards recognizing and categorizing EROs that display changes in behavior between the two stages of data collection (interesting and non-interesting). Oscillations are characterized by their amplitude and phase. The amplitude of an EEG oscillation is typically between 0 and 10 $\mu \mathrm{V}$. According to a classification of different types of oscillatory activity by Galambos there are spontaneous, induced, and evoked rhythms, all of which are differentiated by their response rhythm to the stimulus.

The figure below shows the methodology designed for EEG signal analysis in this project. Raw EEG signal is collected from the subject along with other physiological signals while they watch the two sets of video montages (interesting and non-interesting). Decomposition based approach is used for analysis. EEG signal also requires some preprocessing before being analyzed, so some preprocessing is performed wherein the different fundamental band-components of the signal are extracted.

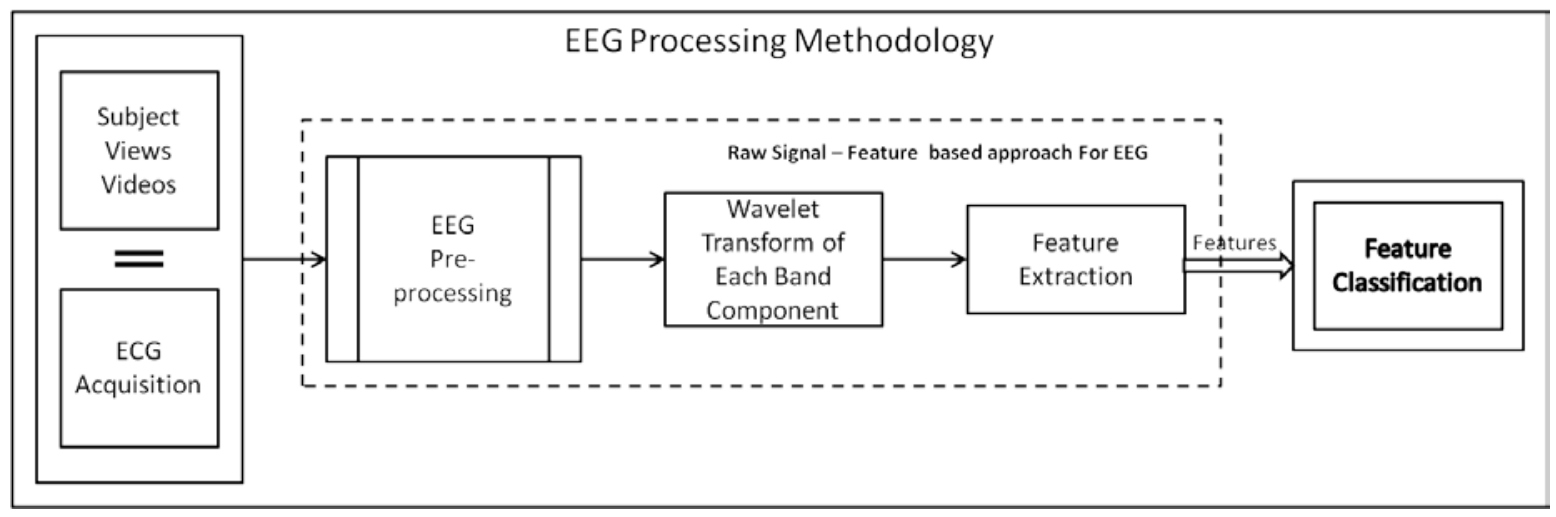

FIGURE 26. EEG PROCESSING METHODOLOGY 
After pre-processing, the different components of the EEG signal are then decomposed using Wavelet Transform. Each of the signal components is decomposed differently in this step based on the core makeup of the signal. After decomposition, the various computed coefficients are then sent through feature extraction step, where various statistical features are computed and assembled for the feature classification step.

Next, the preprocessing and analyses steps have been explained in more detail.

\subsection{Preprocessing of EEG}

The analysis of continuous EEG signals is complex, due to the large amount of information received from every electrode. These to reduce the complexity of the signal information different and somewhat distinct components have been identified which put

together provides the EEG signal itself. These different components have been provided with their own nomenclature, and are categorized by the frequency of their emanations or some cases the shape of their waveform. Although none of these waves is ever emitted alone, the state of consciousness of the individuals may make one frequency range more pronounced than others [129]. 
A typical raw EEG signal during acquisition would look something like the figure below.

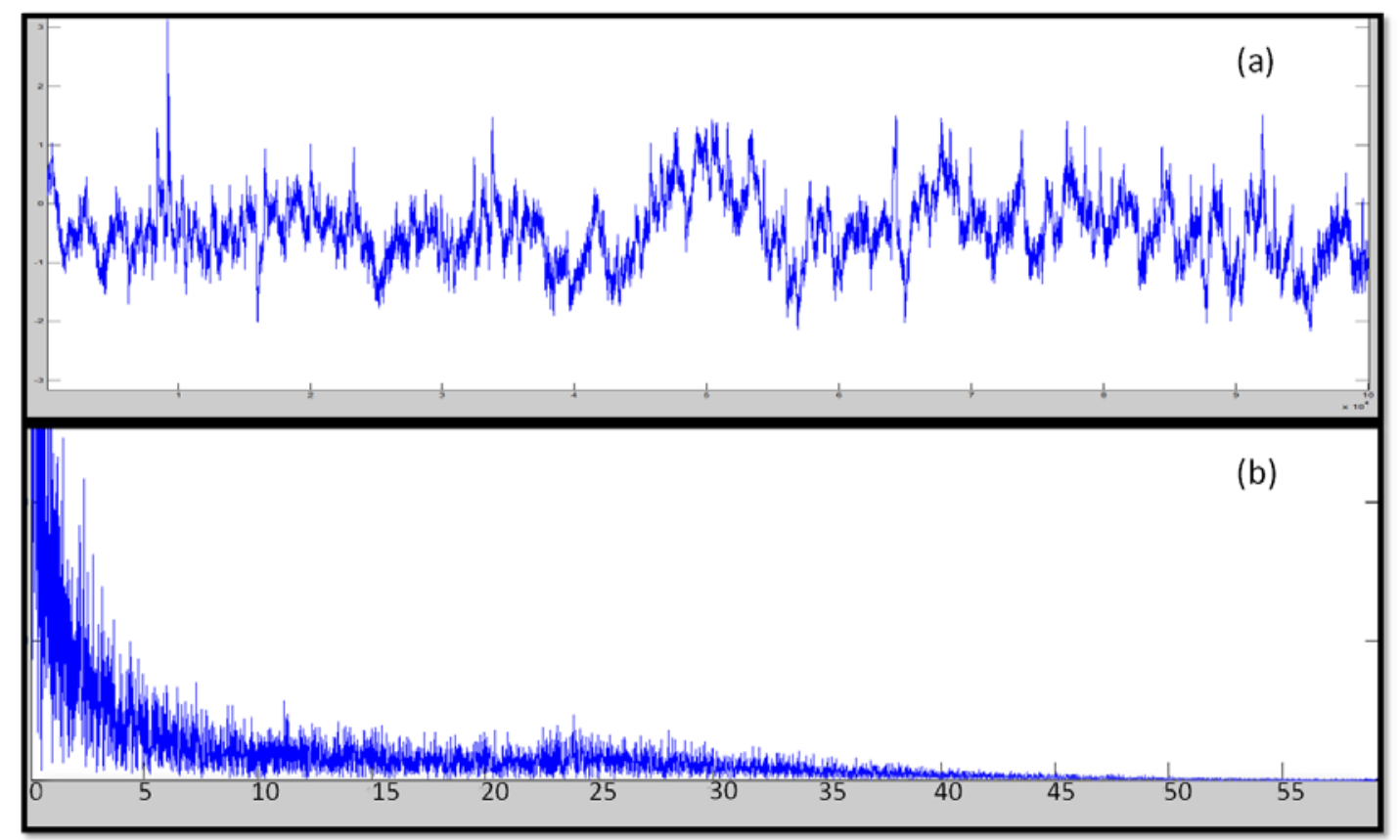

FIGURE 27. (A)RAW EEG SIGNAL (B) EEG FREQUENCY RESPONSE

The different frequency based Band Components of the EEG signal are as follows:

\section{Delta Waves}

Delta waves lie within the range of 0.5 to $4 \mathrm{~Hz}$. Delta waves are primarily associated with deep sleep, and in the waking state, were thought to indicate physical defects in the brain.

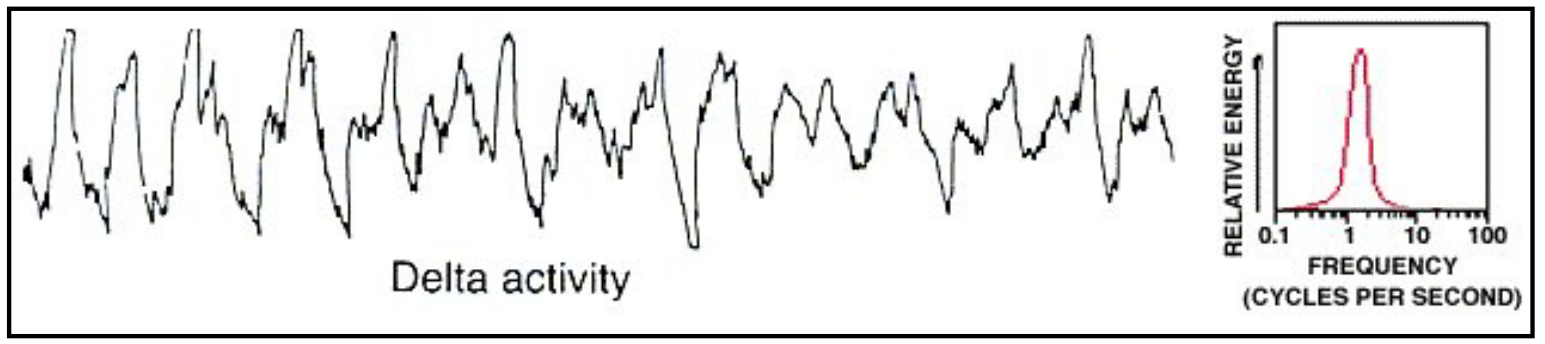

FIGURE 28. DELTA WAVE ILLUSTRATION 


\section{Theta Waves}

Theta waves lie within the range of 4 to $8 \mathrm{~Hz}$, with an amplitude usually greater than $20 \mu \mathrm{V}$. Theta arises from emotional stress, especially frustration or disappointment. Theta has been also associated with access to unconscious material, creative inspiration and deep meditation. The large dominant peak of the theta waves is around $7 \mathrm{~Hz}$.

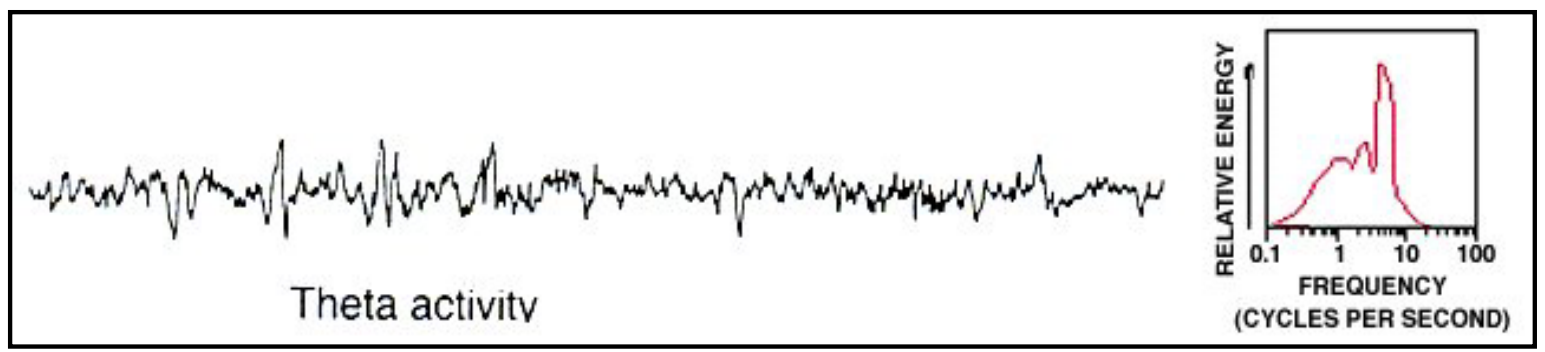

FIGURE 29. THETA WAVE ILLUSTRATION

\section{Alpha Waves}

The rate of change lies between 8 and $13 \mathrm{~Hz}$, with $30-50 \mu \mathrm{V}$ amplitude. Alpha waves have been thought to indicate both a relaxed awareness and also inattention. They are strongest over the frontal (back of the head) cortex and also over occipital cortex. Alpha is the most prominent wave in the whole realm of brain activity and possibly covers a greater range than has been previously thought of. It is frequent to see a peak in the beta range as high as $20 \mathrm{~Hz}$, which has the characteristics of an alpha state rather than a beta, and the setting in which such a response appears also leads to the same conclusion. Alpha alone seems to indicate an empty mind rather than a relaxed one, a mindless state rather 
than a passive one, and can be reduced or eliminated by opening the eyes, by hearing unfamiliar sounds, or by anxiety or mental concentration.

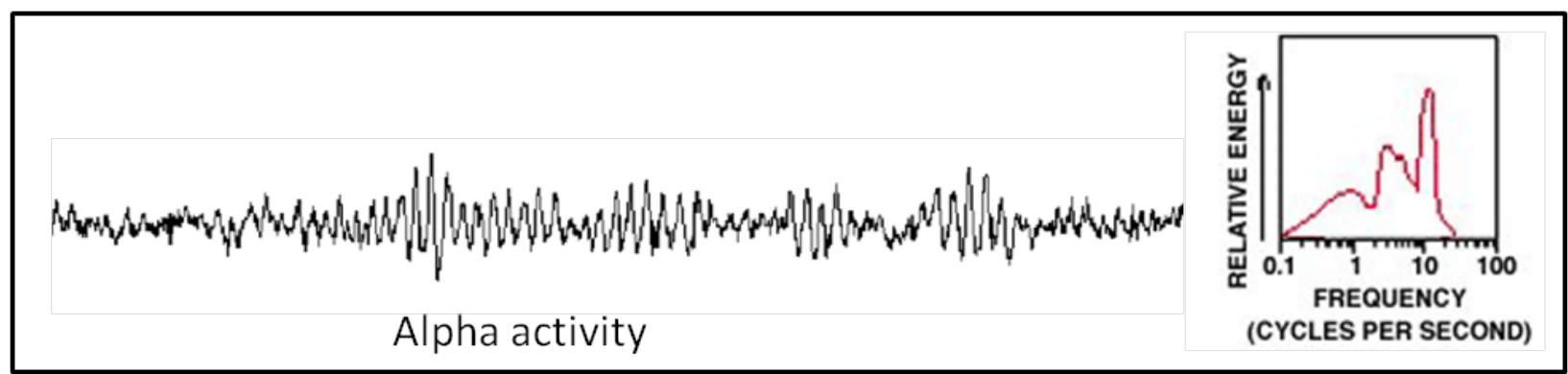

FIGURE 30. ALPHA WAVE ILLUSTRATION

\section{Beta Waves}

The rate of change lies between 13 and $30 \mathrm{~Hz}$, and usually has a low voltage between $5-30 \mu \mathrm{V}$. Beta is thought that this band reflects the mechanism of consciousness that is the binding together of distinct modular brain functions into coherent percepts capable of behaving in a re-entrant fashion.

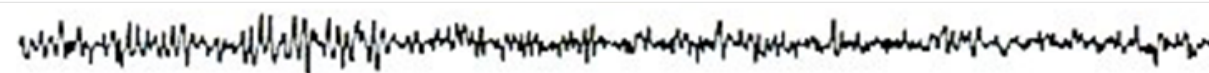

Beta activity

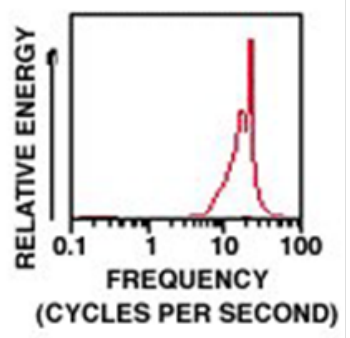

FIGURE 31. BETA WAVE ILLUSTRATION

\section{Gamma Waves}


Gamma waves lie within the range of $35 \mathrm{~Hz}$ and up. is the brain wave usually associated with active thinking, active attention, and focus on the outside world or solving concrete problems.

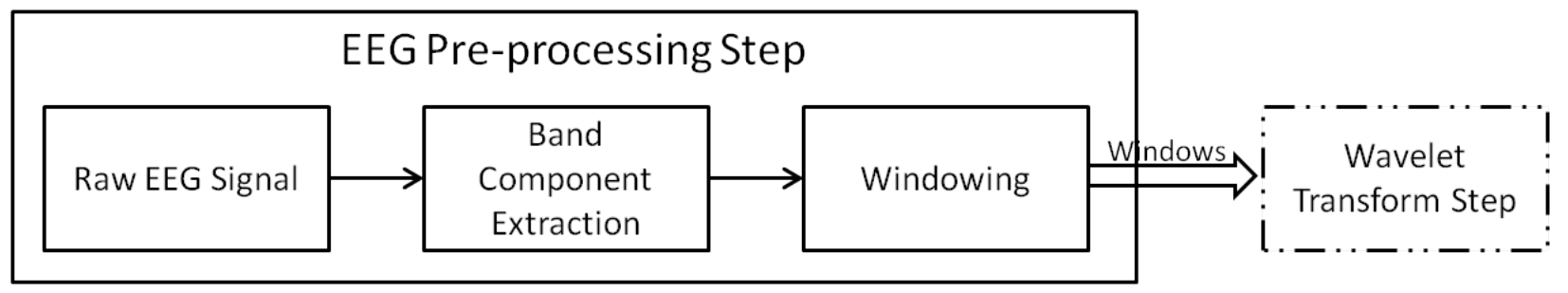

FIGURE 32. EEG PREPROCESSING STEP

To separate these frequency band components from the original EEG signal, a digital filter is required. Since each of the band components lie within specified frequencies, a band-pass filter has been used for the component extractions step. A digital band-pass filter is a time series filter mechanism which allows only certain specified frequency to pass by, while rejecting or attenuating the remaining frequencies of the input signal. There are several different types of band-pass filters that can be used for this purpose. However, in this research the Butterworth filter is used. The Butterworth class of filter was first described in a 1930 paper by the British engineer Stephen Butterworth after whom it is named. The filter response is described by Butterworth polynomials [130]. Butterworth filters are maximally flat, meaning that the response in the frequency domain is the smoothest possible curve of any class of filter of the equivalent order [130]. So using Butterworth filters of order 4, the following frequencies were separated into the various band components of the EEG signal;

- Delta $0.5-4 \mathrm{~Hz}$ 
- Theta $4-8 \mathrm{~Hz}$

- Alpha 8-13Hz

- Beta $13-30 \mathrm{~Hz}$

- Gamma 36-44Hz

Each of these resulting bands is then sent through the 'windowing' step. In this step the interesting and boring portions of the band components based on the timestamps of the original EEG are extracted and sent through a windowing mechanism. In this mechanism, the each band component signal is partitioned into tiny windows. These windows are nonoverlapping and contain ECG data of $10 \mathrm{sec}$ interval. The EEG signal is acquired at a sampling rate of $1000 \mathrm{~Hz} .(1000 \mathrm{~Hz} * 10 \mathrm{sec}=10000$ data-points/window $)$.

Each of the 10 second partitioned window is then decomposed separately in the following step using Discrete Wavelet Transform (DWT).

\subsection{Applying Discrete Wavelet Transform on EEG}

For psychiatry and clinical neuroscience applications, physicians find the ability to study precisely the fundamental dynamics and behavior of the brain's neuroelectirc characteristics very useful. The small yet complex varying frequency structure found in scalp-recorded EEG waveforms contains detailed neuroelectric information about the millisecond time frame of underlying processing systems, and many studies indicate that waveform structure at distinct scales holds significant basic and clinical information [131134]. Small-scale neural rhythms, in particular event related oscillation EROs, have been 
regarded as fundamental to perception and cognition [132]. Wavelet analysis provides a powerful method of isolating such rhythms for study.

There are several applications of Wavelet transform on EEG analysis. It has been used in removal of noise from raw EEG waveforms since wavelet coefficients facilitate the precise noise filtering mechanism by zeroing out or attenuating any coefficients associated primarily with noise before reconstructing the signal with wavelet synthesis [135-137]. Wavelet analysis of EEG has also been extensively used for signal processing applications in intelligent detection systems for use in clinical settings [137, 138]. Wavelet transform has also been used for compression EEG signals, Wavelet compression techniques have been shown to improve neuroelectric data compression ratios with little loss of signal information [136, 139, 140]. It can also been seen that for component and event detection as well as spike and transient detection within the EEG waveforms. Wavelet analysis has proven quite effective in many research studies [138, 140, 141].

As explained earlier in the ECG analysis section, performance of the Wavelet transform depends on the mother wavelet chosen for decomposition of the signal. A common heuristic is to choose one similar to the shape of the signal of interest. So for the set of band components that is extracted from the original EEG signal different mother wavelets that suit different bands are applied during decomposition. Considering the shape and complexity of each band component and by referring published literature, several different mother wavelets were experimented for decomposition in this study. 
For the analysis of the Gamma wave component, the mother wavelet chosen is the 'bior3.9' from the bi-orthogonal family of wavelets. Delta, Theta and Alpha wave components are decomposed using 'db4' as their mother wavelet from the Daubechies family of wavelets. Finally Beta waves are decomposed using 'coif3' as the mother wavelet from the Coiflets wavelet family.

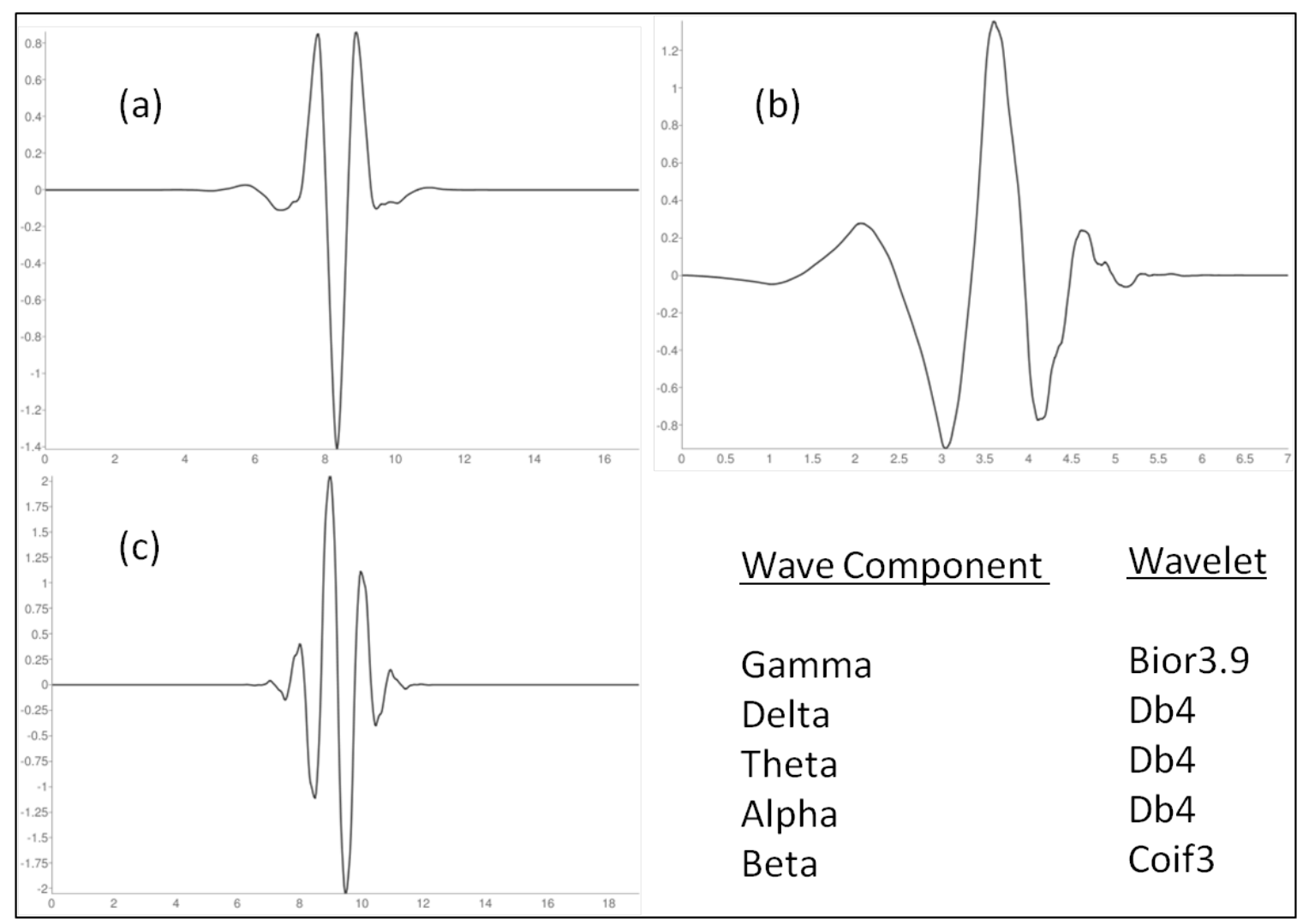

FIGURE 33. (A)‘COIF3’ WAVELET (B) ‘DB4’ WAVELET AND (C) ‘BOIR3.9’ WAVELET.

The decomposition process in wavelet transform can be performed iteratively into several levels. The number of levels chosen for decomposition is application specific and 116 
also depends on the complexity of the signal. For window of the EEG signal band components, 5 levels of decomposition seemed to provide all the required useful information; further decomposition did not yield a better result. The detailed coefficients of all the stages from 1 through 5 and the approximation coefficient of level 5 are retained for feature extraction step.

\subsection{Feature Extraction}

The features computed from these coefficients are as follows: (Here, $\left(\mathrm{x}_{1}, \mathrm{x}_{2}, \ldots \mathrm{x}_{\mathrm{n}}\right)$ represents the values of each coefficient from the 10 sec window)

- Standard Deviation

Equation provided in (8)

- Entropy

Equation provided in (39)

- Log of Variance

Equation provided in (21)

- Mean of frequencies (Fourier domain)

Equation provided in $(24,25)$ 
- Variance of probability distribution

Equation provided in $(27,28)$

- Sum of Auto-Correlation

Equation provided in $(29,30)$

- Mean of Auto-Covariance

Equation provided in $(31,31)$

After the feature extraction has been performed, the total feature set for the wavelet transform step will contain;

6 coefficients $(5$ detailed +1 approximation $) * 7$ (features per coefficient $)=42$ (features columns per band component)

In total there are 5 extracted band components, so,

42 (features per band component) * 5 (different band components) $=210$ (total features from EEG)

These computed features are then sent to the machine learning stage for classification, training and testing. 


\section{CHAPTER 7 Analysis of other physiological signals: Galvanic Skin Response and Heat Flux}

As mentioned earlier the armband is capable of acquiring other physiological signals such as galvanic skin response and het flux. Both these signal are hence collected during the experiment and analysis to see if it yield useful information of the person's level of cognitive attention.

\subsection{Analysis of Galvanic Skin Response}

Skin conductance, also known as galvanic skin response (GSR), psycho-galvanic reflex (PGR), skin conductance response (SCR), electro-dermal response (EDR), or skin conductance level (SCL), is a measure the electrical conductance of the skin. Human skin is a good conductor of electricity, so when a weal electric current is delivered to the skin,

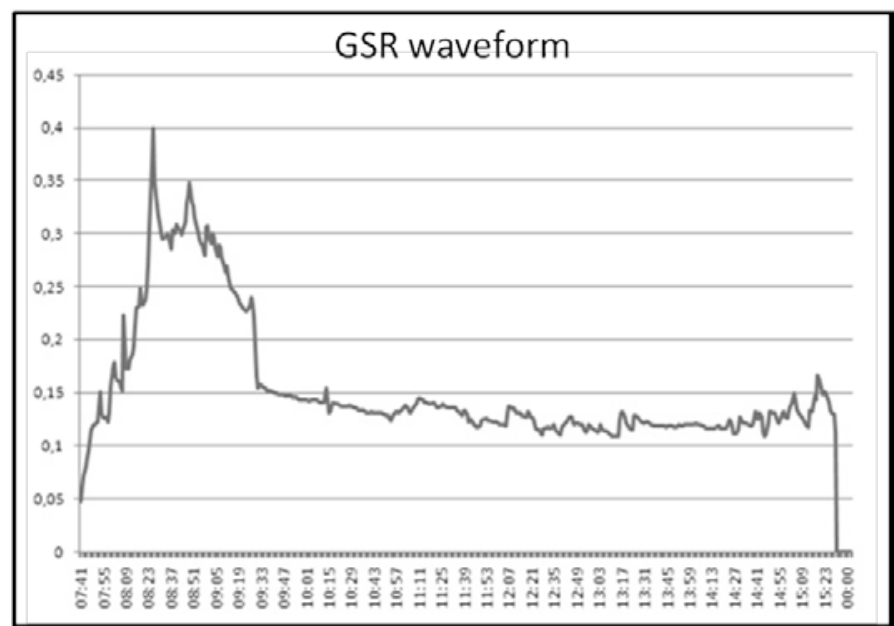

FIGURE 34. GSR WAVEFORM changes in the skin's conduction of that current can be measured. A GSR amplifier applies a constant voltage to the skin through electrodes. In this experiment, the armband has an 
embedded GSR amplifier. The output of the GSR amplifier is the skin's conductance expressed in units called microSiemens $(\mu S)$.

The human skin is an adaptive organ which helps in the maintenance of the body's water balance and temperature. The skin is comprised of three layers, the epidermis, dermis and sub-dermis. Sweat glands which are located in the sub-dermis deposit moisture in the layers of the skin when activated. Sweat glad activation is a simple physiological survival mechanism. The activation is controlled via the sympathetic response of the autonomic nervous system.

The GSR signal is characterized by two types of inherent properties; Tonic and Phasic. Phasic skin conductance refers to the changes in the skin conductance when discrete environmental stimuli like sights, smells, sounds, etc. is presented. Tonic skin conductance is the baseline level of skin conductance. Tonic skin conductance patterns are usually dominant when there is a lack of any particular external stimuli. Tonic skin conductance levels vary over time in individuals depending on his or her psychological state and autonomic regulation. Phasic changes in the skin conductance happens when the sweat glands deposit moisture in the epidermis, and skin conductance returns to normal or tonic levels when the moisture reduces in the layers of the skin [142, 143].

Typically the different features that can be extracted and quantified from GSR signals are amplitude (in $\mu S$ ), latency, rise time, and half recovery time. The amplitude of a GSR at any given time can either be the absolute reading of the amplifier or the difference between the tonic skin conductance level at the time the response was evoked and the skin 
conductance at the peak of the response. Latency Is the time period between when the stimulus was produced and when the response is recognized on the GSR signal. Latency values are usually 3 seconds or less. Rise time is the difference in time between the onset of the response in GSR and the peak of the recorded response. Typical values of rise time are 1 to 3 seconds. Half recovery time is the time between the peak of the response and the point in the signal when the conductance returns to amplitude that is half of the peak amplitude.

The GSR data that was collected from the armband for this experiment turned out to be low frequency of $32 \mathrm{~Hz}$. Although usually despite the low frequency the information could perhaps have been useful for analysis, but in this case each subject's data after acquisition seemed to provide very little evidence of information. After a thorough investigation and going back and forth with the manufacturers of the armband it was realized that due to the sedentary nature of the experiment conducted wherein the subject barely moves and has no physical activities, the armband was not able to pick up the subtle changes in GSR. Hence it collected no information of use. This was primarily because the armband's GSR sensor was designed to collect data best during physical exercise which results in increased moisture content in the skin due to sweating. Since the subject of this experiment did not have any physical activities there was no large changes in the moisture content on their skin. Hence for our future work it is highly recommended that a different GSR sensor needs to be used which has large fidelity high frequency sensors that are capable of acquiring useful galvanic conductance information of the skin even during little 
to no physical activity by the subject. Due to these issues found with the GSR data, it is not going to be included in the scope of this research thesis. However, due to the extensive work in algorithm development specifically for GSR data that has already been developed, it would be rather easy and straight forward to include GSR data into the analysis once the right data acquisition sensors are available.

\subsection{Analysis of Heat Flux}

Temperature is used as one of the most common indicator of a person's well being, since many medical conditions directly affect the core body temperature. Heat Flux [HF] is the amount of heat that the body emits. Studies have shown that Heat Flux is effective in detecting switches in emotional and/or physical contexts. Physical movements and sometimes emotional changes causes the body to warm up and therefore emit heat. Different environmental conditions can also have an effect on Heat Flux [143].

The proprietary heat flux sensor being used in the armband is a robust and reliable device that reflects a measure of the amount of heat being dissipated by the body. The sensor uses very low thermally resistant materials and extremely sensitive thermocouple arrays. It is placed in a thermally conductive path between the skin and the side of the armband exposed to the environment. A high gain internal amplifier is used to bring the

signal to a level that can be sampled by the microprocessor located in the SenseWear Armband. 
Skin temperature is also measured using a highly accurate thermistor-based sensor located on the backside of the armband near its edges and in contact with the skin. Continuously measured skin temperature is reflective of the body's core temperature activities. The near-body ambient temperature sensor measures the air temperature immediately around the wearer's armband. This sensor also uses a highly accurate thermistor-based sensor and directly reflects the change in environmental conditions around the armband; for example, walking out of an air-conditioned building on a hot day.

Studying the changes in the heat dissipation of the body or heat flux could be another source of information in recognizing the attention levels of an individual.

For this study the HF data collected from the armband was at $32 \mathrm{~Hz}$. Since it was a low frequency signal the signal is used as is and no noise filtering is performed on it in fear of losing what little information it might contain. So the raw HF signal is partitioned using the same windowing mechanisms as used before where 10 seconds non-overlapping windows are used and each of the extracted windows are sent through feature extraction step. Just as in the other analysis aspects, several statistical features are extracted such as Standard Deviation, Entropy, power, Energy, Skewness, Kurtosis etc. Totally 18 different features are extracted per window of the HF signal.

These computed features are then sent to the machine learning phase for checking the accuracy of classification. 
As it turns out, with by-subject validation across all available HF data (18 subjects), the accuracy was around 53\% with random forest method used for classification. Due to the low overall accuracy and performance statistics of HF signal, it has not been included in the studies final results which is provided in the results section. The causes for HF to underperform might partly be because of the low sampling rate of the HF signal acquired and partly because the experiment lacked any physical activities. HF signal is more informative for analysis of experimental conditions where physical activities might be involved. 


\section{CHAPTER 8 MACHINE LEARNing AND ClassificATION MOdEL}

The Machine learning paradigm has become an essential and significant aspect of today's computing. Machine learning methods and algorithms are being increasing used to solve complex problems and applications of this are being integrated more and more into our daily lives. Generally speaking, machine learning deals with the design of mathematical constructs for computers that in essence mimic the fundamental learning functionalities of humans. These mathematical constructs are designed to learn rules from data, adapt to changes, and improve performance of a given program. Many of the programs that are developed today are tackling highly complex problems that cannot be explicitly described to the computer. These problems such as identifying faces in images, autonomous driving, drug interaction discovery, finding patterns in large volumes of scientific data, performance optimization of systems etc cannot be cannot be described or delineated just with a set of rules. Hence to solve such vast and complex problems with the use of computers, the fundamental principles of learning itself has to be embedded into the programs of today as a computation process. This type of embedding machine learning aspects to computational solution development is known as computational learning theory.

A famous definition of Machine learning by Tom M. Mitchell says that 'it is a computer program that is said to learn from experience $\mathrm{E}$ with respect to some class of 
tasks $\mathrm{T}$ and performance measure $\mathrm{P}$, if its performance at tasks in $\mathrm{T}$, as measured by $\mathrm{P}$, improves with experience E' [144]. What that means is that machine learning algorithms capture certain key characteristics of unknown underlying probability distributions within a given dataset. Recognizing such characteristics within the data allows the program to predict, categorize and classify new and unseen yet similar input data.

The taxonomy of machine learning algorithms can be organized based on the type of outcome the algorithm provides. There is supervised learning type where functions that map input variables a desired and specified output using a labeled training set, after which it predicts on unlabeled data set which is the testing set. Unsupervised learning models a set of inputs to clusters during training. Semi-supervised learning combines both supervised and un-supervised learning models. Reinforcement learning is a type of machine learning where the learning mechanism includes a feedback in the form of rewards or weights. Transduction type of machine learning tries to predict new outputs based on specific and fixed cases of observations. Finally the learning to learn type of machine learning learns from its own inductive bias based on previous experiences.

Over the years several different approaches have been developed in machine learning such as decision tree learning, association rule learning, genetic or evolutionary computing, artificial neural networks, support vector machines, clustering etc. All of these are fundamentally modeled upon the functionalities of human learning mechanisms. The use of specific machine learning depends highly on the nature of application. 
In this application the result after signal processing on acquired psychological signals is a large set of features. Since the data was collected in a systematic and controlled environment, the features extracted from respective portions of the signals can be classified under the two presumed categories: 'attention' and 'non-attention'. Hence supervised learning method is used for this study to developed classification heuristics.

\subsection{Feature Space Reduction}

While dealing with data that has large number of features one can encounter certain issues while using these features to develop classification model. This is especially true for some machine learning methods where large feature spaces can be a hindrance from obtaining useful classification models.

Given the different methods of analysis performed on the ECG signal, the combined feature set turns out to be a very large. To emphasize we have:

- 157 features from HRV and its related values

- 27 features from Stockwell Transform

- 210 features from Dual Tree Complex Wavelet Transform

So in total by combining all the features computed from ECG signal analysis we get 394 features.

From EEG analysis, a total of 210 features are obtained. These features are computed for each window. There by forming several instances of such features for a given dataset. So before sending these features for developing and testing a classification 
model, it is important to identify those features that are most useful in providing information towards classification and decision making. To do this 'info-gain' method is used. Info-gain is a measure of impurity which computes an index for each feature based on the quality of information it provides to classify the instance between the two labeled classes. In this case the label of 'attention' and 'no-attention'. The following is the computation equation behind info-gain method.

$$
\begin{gathered}
\text { Measure of Impurity } \\
\text { InfoGain: } I(v)=-\sum_{i=0}^{c-1} p\left(\frac{i}{v}\right) \log _{2} p\left(\frac{i}{v}\right) \quad \text { (65) } \\
\text { Where; } \\
\mathrm{I}(\mathrm{v})=\text { impurity measure of feature ' } \mathrm{v} \text { '. } \\
\mathrm{p}(\mathrm{i} / \mathrm{v})=\text { proportion of observation from class ' } \mathrm{i} \text { ' at feature ' } \mathrm{v} \text { '. } \\
\mathrm{C}=\text { number of classes }
\end{gathered}
$$

By obtaining the indexes for each of the features in the feature table, the features can be ranked in the order of most informative towards decision making between the classes. The info-gain indices are obtained across all subjects where a by-subject feature ranking is performed. In this, in each iteration a particular subject's feature set is considered as the test set while the remaining subjects feature sets are pooled together to form the training set. These test and training sets will be used towards machine learning model development. Using the feature set of each respective subject, the feature ranking is performed using 
info-gain measure. After obtaining the info-gain indices, the features are ranked based on the value of its index informing the quality of the feature. Using these ranking across all subjects the top 10, 20, 3040 etc features are used towards development of the classification models. This allows for the selection of the least number of features required to obtain the highest form of classification accuracies. The next step is to apply these selected features using a machine learning method.

\subsection{Random Forest}

There are several machine learning algorithms to choose from, where the choice simply depends on the type of dataset and its complexity.. Although several machine learning algorithms such as C4.5, Support Vector Machine, Classification via Regression etc were experimented with, it consistently was proven than with the kind of features that is being dealt with in this study 'Random Forest' based classification method worked best, outperforming the rest mentioned classifiers every time.

Leo Breiman developed random forest classification method which is basically an ensemble classifier that consists of multiple decision trees [150]. It is a very accurate classifier which displays great success with multiple datasets. It is especially useful with data mining extremely large datasets and databases. Unlike the other tree-based classifiers random forest uses multiple trees or a forest to develop decisions and classifications. Although in this study it is being used to develop models based on supervised data, random forest can be used for unsupervised data learning as well [151]. Random forest has been popular for applications in bio-signal and bio-medicine [152]. 
In the Random Forests method many classification trees are grown to develop the rules for decisions and classifications. To classify a new object from an input vector, the input vector is put down each of the trees in the forest. Each tree gives a classification, and the tree "votes" for that class. Over all the grown tress, the forest chooses the classification having the most votes. When the training set for the current tree is drawn by sampling with replacement, about one-third of the cases are left out of the sample. This OOB (out-of-bag) data is used to get a running unbiased estimate of the classification error as trees are added to the forest. It is also used to get estimates of variable importance. After each tree is built, all of the data are run through the tree, and proximities are computed for each pair of cases. If two cases occupy the same terminal node, their proximity is increased by one. At the end of the run, the proximities are normalized by dividing by the number of trees. Proximities are used in replacing missing data, locating outliers, and producing illuminating lowdimensional views of the data.

In random forests, there is no need for cross-validation or a separate test set to get an unbiased estimate of the test set error. It is estimated internally, during the run. To do this, each tree is constructed using a different bootstrap sample from the original data. About one-third of the cases are left out of the bootstrap sample and not used in the construction of the kth tree. Then each case that is left out is then put in the construction of the kth tree to get a classification. In this manner, a test set classification is obtained for each case in about one-third of the trees. At the end of the run, $\mathrm{j}$ is taken to be the class that 
gets most of the votes every time case $n$ was OOB. The proportion of times that $\mathrm{j}$ is not equal to the true class of $n$ averaged over all cases is the OOB error estimate.

Each tree is grown as follows: If the number of cases in the training set is $\mathrm{N}$, sample $\mathrm{N}$ cases at random - but with replacement, from the original data. This sample will be the training set for growing the tree. If there are $\mathrm{M}$ input variables, a number $\mathrm{m}<<\mathrm{M}$ is specified such that at each node, $\mathrm{m}$ variables are selected at random out of the $\mathrm{M}$ and the best split on these $\mathrm{m}$ is used to split the node. The value of $\mathrm{m}$ is held constant during the forest growing. Each tree is grown to the largest extent possible. There is no pruning.

Every time a split of a node is made on variable $m$ the gini impurity criterion for the two descendent nodes is considered less than the parent node.

Gini Measure of Impurity:

$$
\begin{gathered}
\text { Gini: } I(v)=1-\sum_{i=0}^{c-1} p\left(\frac{i}{v}\right)^{2} \\
\text { Where; } \\
\mathrm{I}(\mathrm{v})=\text { impurity measure of feature ' } \mathrm{v} \text { '. } \\
\mathrm{p}(\mathrm{i} / \mathrm{v})=\text { proportion of observation from class ' } \mathrm{i} \text { ' at feature ' } \mathrm{v} \text { '. } \\
\mathrm{C}=\text { number of classes }
\end{gathered}
$$


Adding up the gini index decreases for each individual variable over all trees in the forest gives a fast variable importance that is often very consistent with the permutation importance measure.

Random forest classification method is famous for its efficiency on large datasets. It has an effective way of estimating missing data and maintains accuracy when a large proportion of the data is missing.

\subsection{Parameter Selection for Random Forest}

The output characteristics of random forest implementation can vary based on certain parameters that random forest uses. These parameters need to be chosen such that the best classification output can be achieved for a given input dataset. These parameters are number of trees and input seed. Here an ensemble random forest classifier has been designed; where in several random forests are used to provide the final classification output. Using the classification output from each of the random forests a probability based average voting method is used to compute a single ensemble output. By default the random forest implementation uses the first feature column as the seed input. However since here many forests are being used, different feature columns are supplied as seed values. This allows each forest to grow uniquely based on the seed feature. Thereby making the ensemble output more generalized across all features. The two parameters of the ensemble 
random forest that are tuned here is number of trees in each forest and the number of forest.This ensemble classifier has been nicknamed as 'Forest of Forest'.

Number of trees: Typically the number of trees that are grown inside the random forest implementation is limited to a default number in random forest implementation. Having a fixed number of trees for varying number of features during implementation may not always provide the best classification accuracies. Hence, in this study the number of the trees is computed dynamically based on the number of features that is input as the feature set. This computation is done as follows:

$$
\text { Number of trees }=\text { ceil }(10+\text { NumberOfFeatures } / 10)
$$

Where ceil is the operation of rounding the number off to the next integer value.

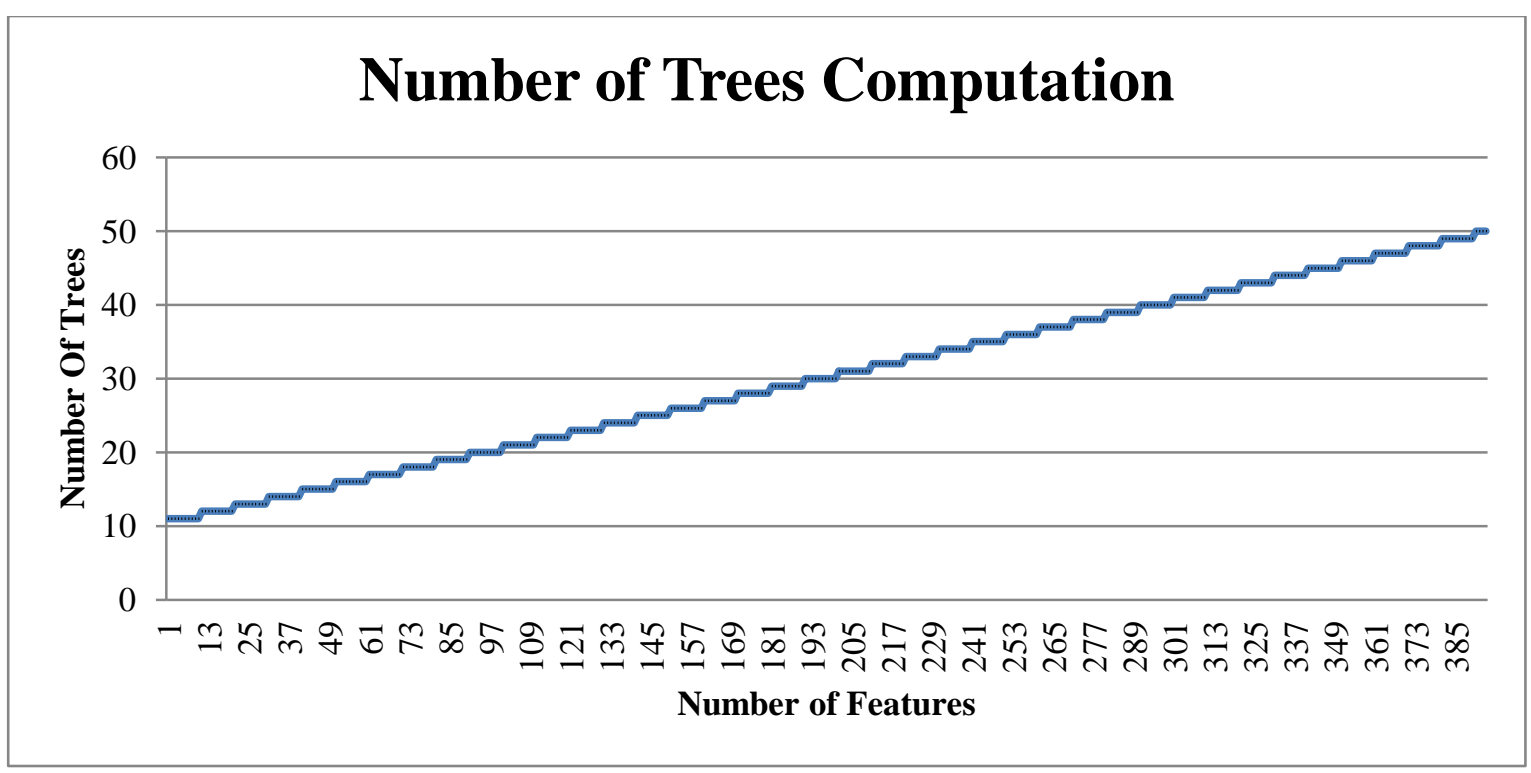

FIGURE 35: DYNAMIC COMPUTATION OF NUMBER OF TREES 
A controlled linear increase has been applied in this case so as to have a control over the number of trees that are used for the random forest implementation. Having either a fixed number of trees or a same number of trees as the features is not a good way of implementing random forest especially when varying sizes of features are being experimented with.

Number of forests: Typically the depth of trees that are grown inside the random forest implementation is limited to a default number in random forest implementation. Having a fixed number of forests for varying number of features during implementation may not always provide the best classification accuracies. Hence, in this study the number of forests is computed dynamically based on the number of features that is input as the feature set. This computation is done as follows:

$$
\text { Number of Forests }=\operatorname{ceil}(2 * \log (\text { NumberOfFeatures }))
$$

Where ceil is the operation of rounding the number off to the next integer value. 


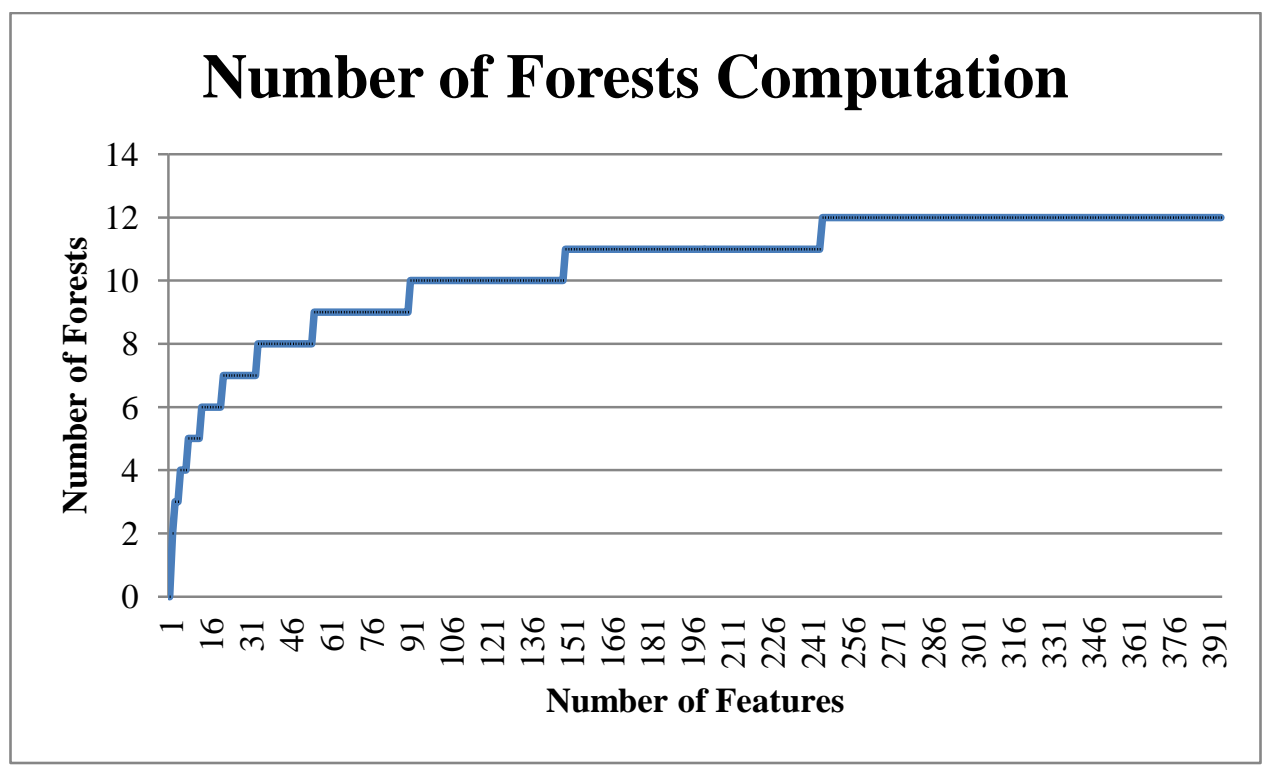

FIGURE 36: COMPUTATION OF DEPTH OF TREES DYNAMICALLY

A logarithmic scale has been implemented to control the number of forests being used for developing models for varying sizes of feature sets. Since neither fixed nor uncontrolled number of forests are particularly useful in practical applications of random forest with varying number of features in the data set. This allows a more controlled development of the overall ensemble model.

This dynamic parameter selection has been implemented for the combined feature set analysis of ECG and the results have been discussed in the following results section. 


\section{CHAPTER 9 RESULTS}

After every analysis described in this manuscript, the features computed from each of them is fed to the machine learning stage. In the machine learning step, although various classifier methods such as C4.5, Classification via Regression, SVM etc. were tested and in turn it was realized that 'Random Forest' classifier worked the best for our purpose, hence for developing the classifier we used random forest classification method with parameters as suited. For developing the classification model for each of the classifier 'by-subject' or 'leave one subject out' based training and test sets are prepared. In this type of training and testing, out of the given number of subject say $x, x-1$ subjects are subjects are used for training and developing the classification model, while the xth subject's data is used for testing the developed model. This procedure is repeated in a round robin fashion until each of the subject's data in the total collected data has been tested with a classification model developed exclusively for it. So for each type of classification method, the average accuracies and other statistics have been presented over all the subjects.

The output of all classification is obtained first in the format of a confusion matrix.

TABLE 1. CONFUSION MATRIX TEMPLATE

\begin{tabular}{|c|c|c|}
\hline Confusion Matrix & attention & Non-attention \\
\hline attention & True Positives & False Positives \\
\hline Non-attention & False Negatives & True Negatives \\
\hline
\end{tabular}


All the calculations presented hence forth stem from this output of the confusion matrix.

Accuracy is simply calculated by

$$
\text { Accuracy }=\frac{\text { truePositives }+ \text { trueNegetives }}{\text { total number of instances }}
$$

The classification sensitivity and specificity calculated as follows:

$$
\begin{aligned}
& \text { Sensitivity }=\frac{\text { truePositives }}{\text { truePositives }+ \text { falseNegative }} \\
& \text { Specificity }=\frac{\text { trueNegetives }}{\text { trueNegetives }+ \text { falsePositives }}
\end{aligned}
$$

In the following subsections, various formats of the computed results have been presented. First the results from individual computation methods have been show to understand the effectiveness and contribution of each of these methods towards the overall goal. Next the results from the combined feature set has been provided where all available ECG data and EEG data has been utilized. Following which a comparison analysis is provided wherein the analysis from the corresponding ECG and EEG is presented for the relevant subject cases. Finally analysis results that is performed based on design towards practical application is provided. 


\subsection{Results from Individual Analysis Methods}

Since the project encompasses several different and distinct mechanisms in signal processing to analyze the given signals, it is important to understand how much each of these analysis methods contribute to the overall projects output. To do this, in this section the features extracted from each of the signal processing mechanisms applied earlier are individually applied to machine learning paradigm and the resulting outputs are discussed. The classification methods used is random forest, in total there are 23 ECG datasets and 15 EEG datasets.

As mentioned earlier, the application of Discrete Wavelet Transform (DWT) alone did not yield satisfactory results especially in comparison with HRV feature analysis and ST feature analysis. Hence an alternate format of wavelet based method called Dual Tree Complex Wavelet Transform (DTCWT) was applied by which the results seem to improve vastly. The following is a comparison of the previously obtained output from DCT and the results obtained from DTCWT.

\subsubsection{Results of Discrete Wavelet Transform}

Using the Random Forest Classification method, the accuracies of the classification on the feature set obtained by DWT analysis alone on by-subject test data set with respective training sets are as follows; 
TABLE 2: DWT CLASSIFICATION ACCURACIES BY SUBJECT

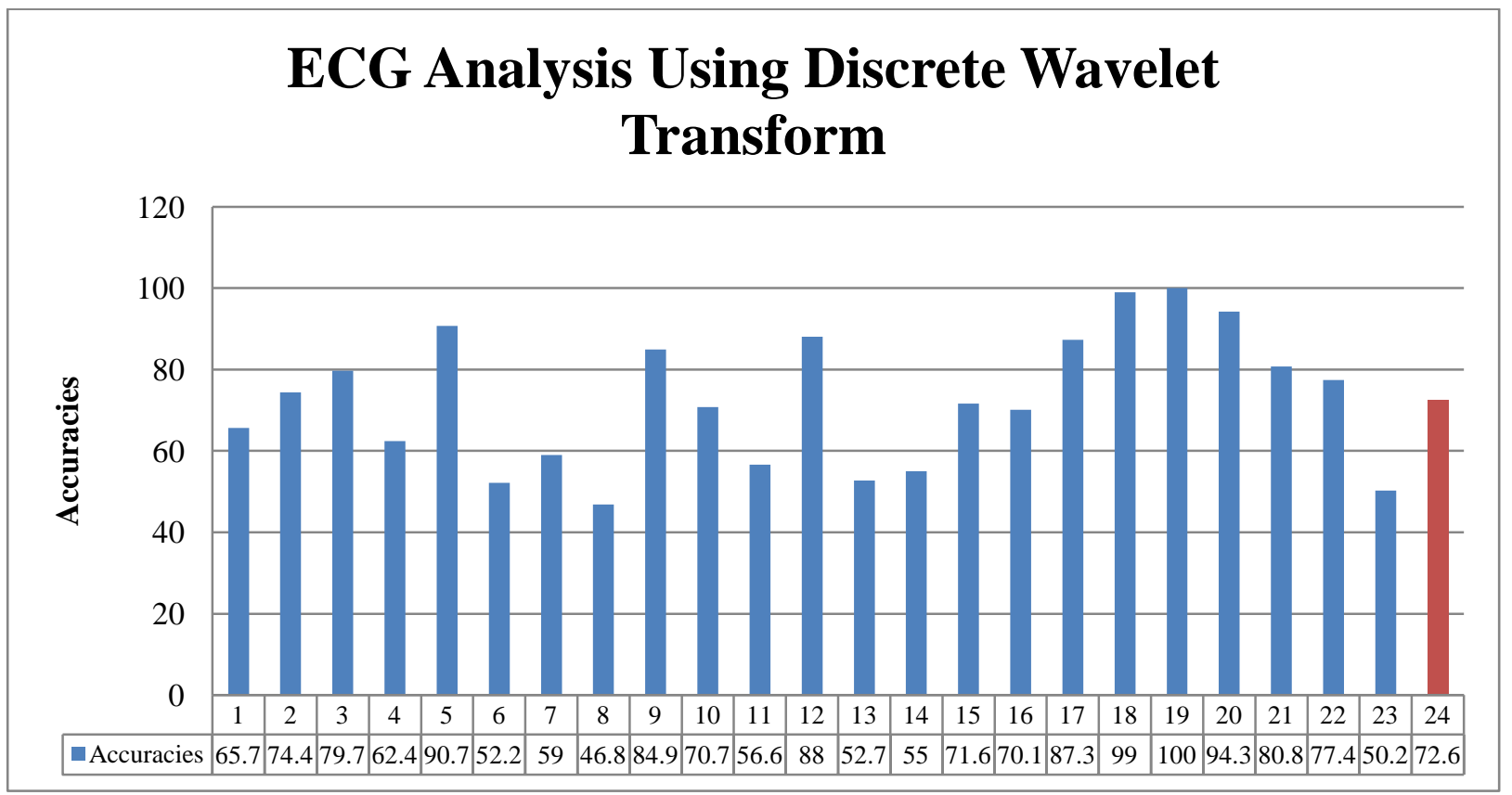

In the table columns 1-23 shows the different accuracies obtained from each by-subject validation for each subject. The mean accuracy of features is $72.6 \%$, which is shown in the table as the red bar on the $24^{\text {nd }}$ column. The mean specificity of the classification over all subjects is $63.20 \%$ and the overall average sensitivity of the classification on DWT features of ECG is $81.98 \%$. 


\subsubsection{Results of Dual Tree Complex Wavelet Transform (DTCWT)}

Using the Random Forest Classification method, the accuracies of the classification on the feature set obtained by DTCWT analysis on by-subject test data set with respective training sets are as follows;

TABLE 3: DTCWT CLASSIFICATION ACCURACIES BY SUBJECT

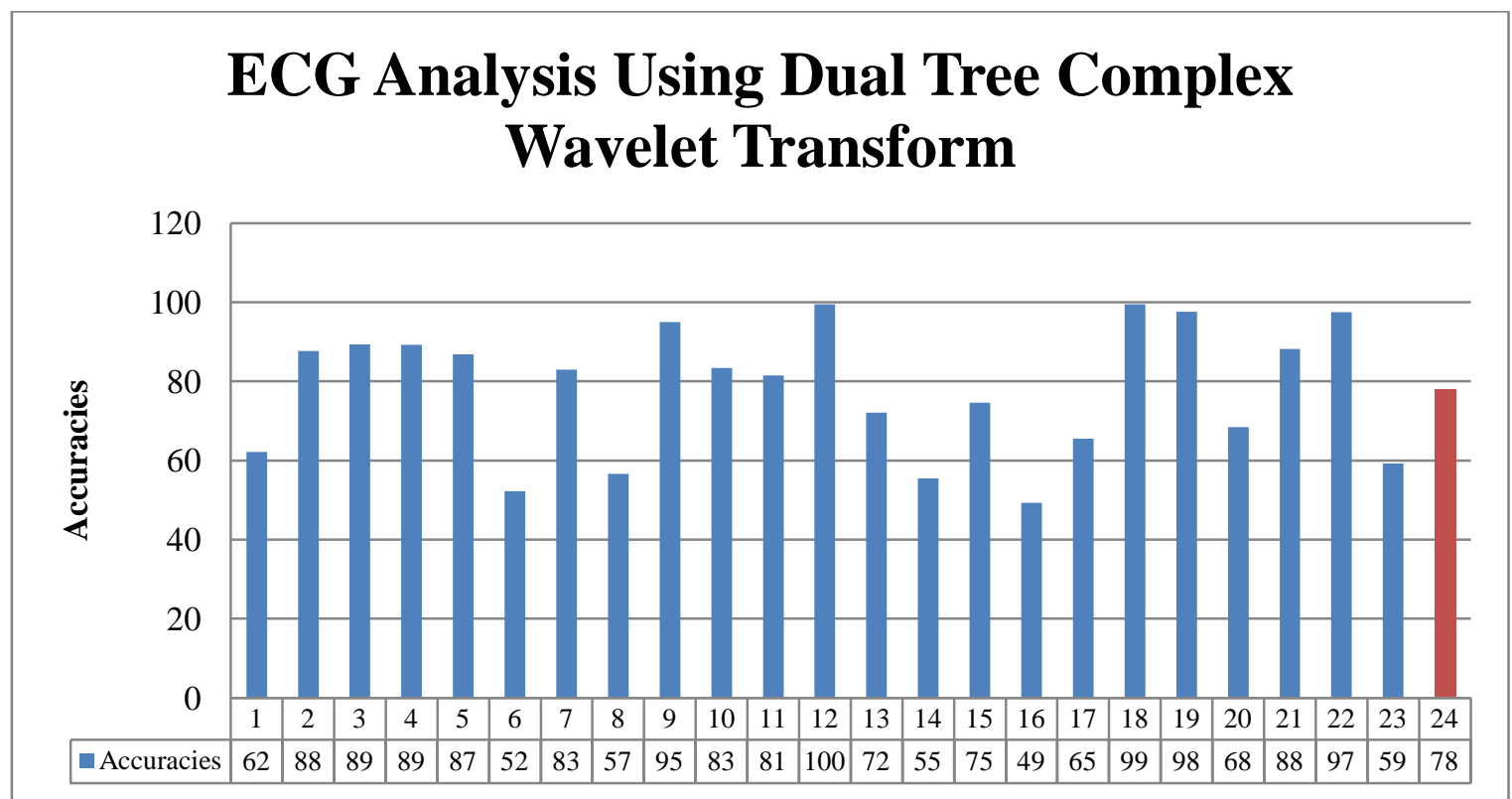

In the table columns 1-23 shows the different accuracies obtained from each by-subject validation for each subject. The mean accuracy of features is $77.98 \%$, which is shown in the table as the red bar on the $24^{\text {nd }}$ column. The mean specificity of the classification over all subjects is $68.88 \%$ and the overall average sensitivity of the classification on DCTWT features of ECG is $87.08 \%$. 
As it can be seen, the mean accuracy of ECG analysis using Discrete Wavelet Transform was around $73 \%$ while the mean accuracy of ECG analysis using Dual Tree Complex Wavelet Transform was around 78\%, which a overall increment of about 5\%. With regards to the sensitivity and specificity as well, the DTCWT seemly outperforms DWT. Since DTCWT shows significant improvements in results as compared to DWT, the former has been employed in the final analysis while the later method has not been used.

\subsubsection{Results of Stockwell Transform (ST)}

Using the Random Forest Classification method, the accuracies of the classification on the feature set obtained by ST analysis on by-subject test data set with respective training sets are as follows;

TABLE 4: S TRANSFORM CLASSIFICATION ACCURACIES BY SUBJECT

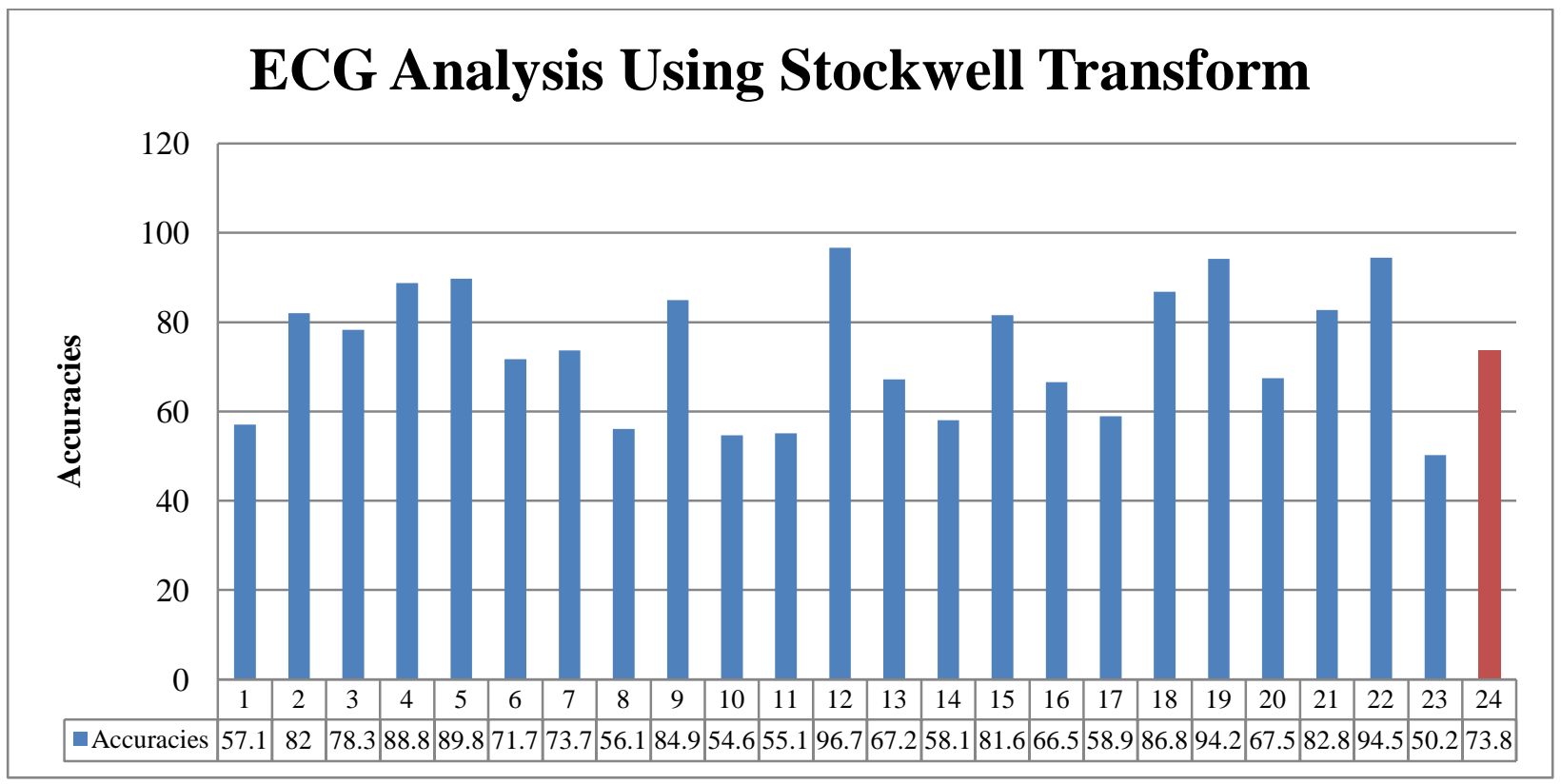


In the table columns 1-23 shows the different accuracies obtained from each by-subject validation for each subject. The mean accuracy of features is $73.77 \%$, which is shown in the table as the red bar on the $24^{\text {nd }}$ column. The mean specificity of the classification over all subjects is $66.83 \%$ and the overall average sensitivity of the classification on ST features of ECG is $80.96 \%$.

\subsubsection{Results of Heart Rate Variability (HRV) and Related Values}

Using the Random Forest Classification method, the accuracies of the classification on the feature set obtained by HRV analysis on by-subject test data set with respective training sets are as follows; 
TABLE 5: HRV VALUES CLASSIFICATION ACCURACIES BY SUBJECT

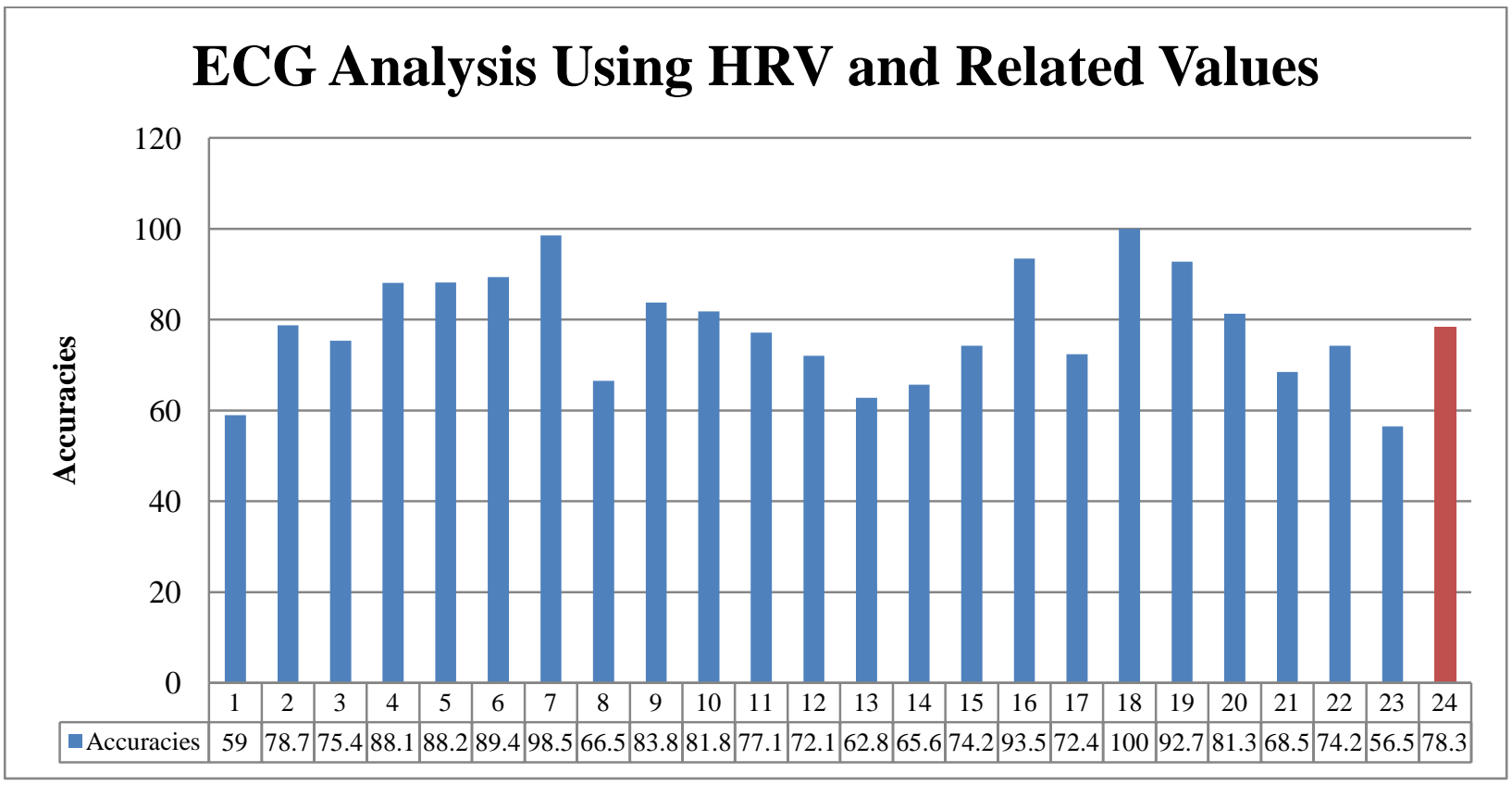

In the table columns 1-23 shows the different accuracies obtained from each by-subject validation for each subject. The mean accuracy of features is $78.27 \%$, which is shown in the table as the red bar on the $24^{\text {nd }}$ column. The mean specificity of the classification over all subjects is $69.80 \%$ and the overall average sensitivity of the classification on HRV features of ECG is $86.79 \%$.

\subsubsection{Results of combined feature set of ECG analysis}

The features obtained from each of the ECG analysis methods namely, DTCWT, HRV, ST, are combined to form the total feature extraction process. The classification accuracy obtained from the combination of these feature sets provide the complete picture with respect to the accuracy of classification obtained by this research on ECG. 
Using the Random Forest Classification method, the accuracies of the classification on the feature set obtained by HRV analysis on by-subject test data set with respective training sets are as follows;

TABLE 6:COMBINED FEATURESET OF ECG CLASSIFICATION ACCURACIES BY SUBJECT

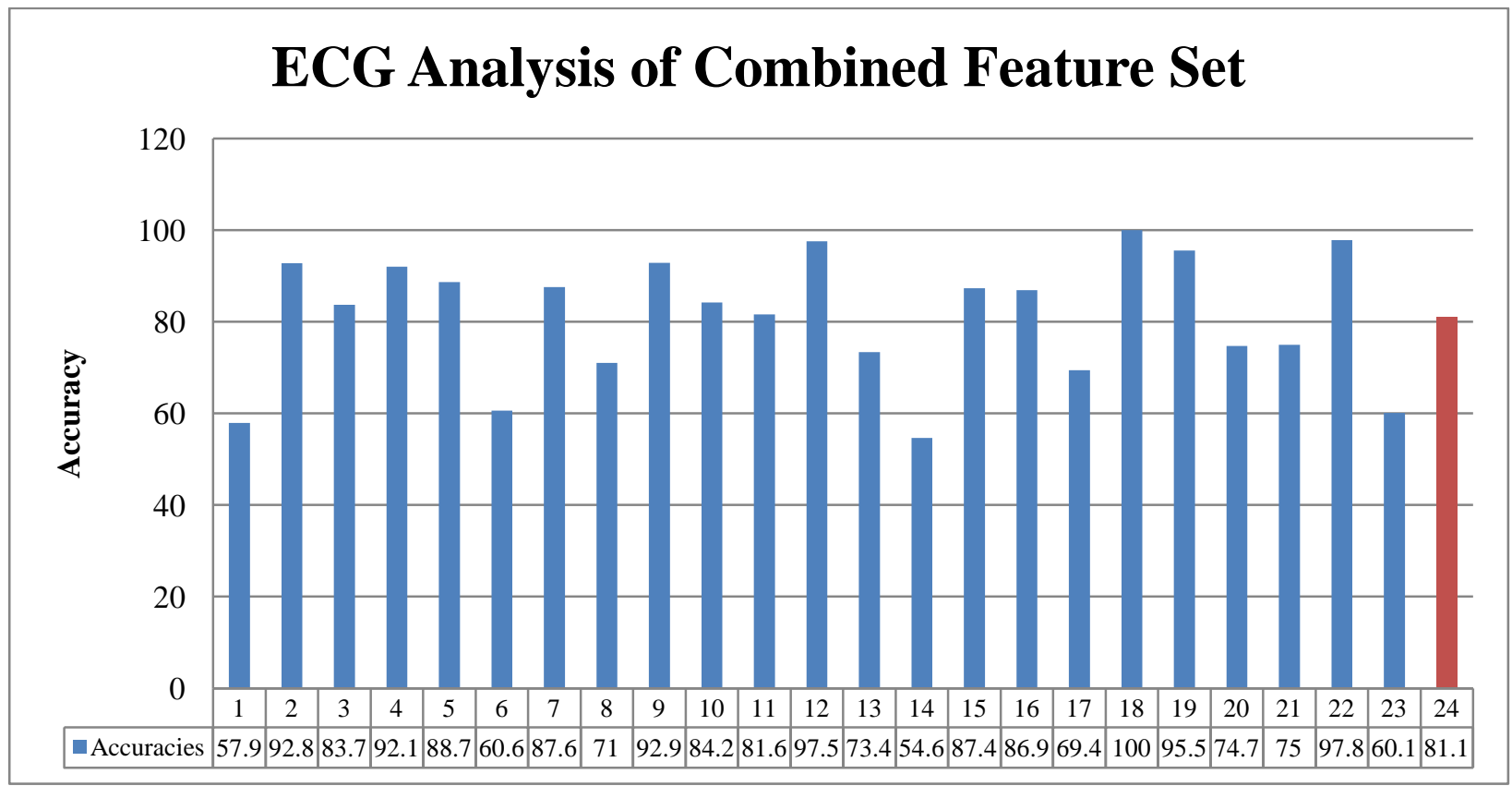

In the table columns 1-23 shows the different accuracies obtained from each by-subject validation for each subject. The mean accuracy of features is $81.11 \%$, which is shown in the table as the red bar on the $24^{\text {nd }}$ column. The mean specificity of the classification over all subjects is $74.78 \%$ and the overall average sensitivity of the classification on combined feature set of ECG is $87.74 \%$.

It can be seen that the mean accuracy of classification of the entire feature set is $81.11 \%$ which is a great improvement than classification results obtained from any one analysis 
method alone. Also the values specificity and sensitivity has become greater than those obtained from individual methods, thereby providing a more robust classification. So combing different signal analysis methods helped improve the classification between cases of attention and no-attention using ECG.

\subsubsection{Results of feature set from EEG analysis}

Since EEG data is being used to compare the accuracy of classification of ECG analysis, feature set from discrete wavelet transform analysis of EEG is obtained. There are in total 15 datasets available for EEG.

Using the Random Forest Classification method, the accuracies of the classification on the feature set obtained by DWT analysis on by-subject test data set with respective training sets of EEG are as follows; 
TABLE 7: EEG ANALYSIS CLASSIFICATION ACCURACIES BY SUBJECT

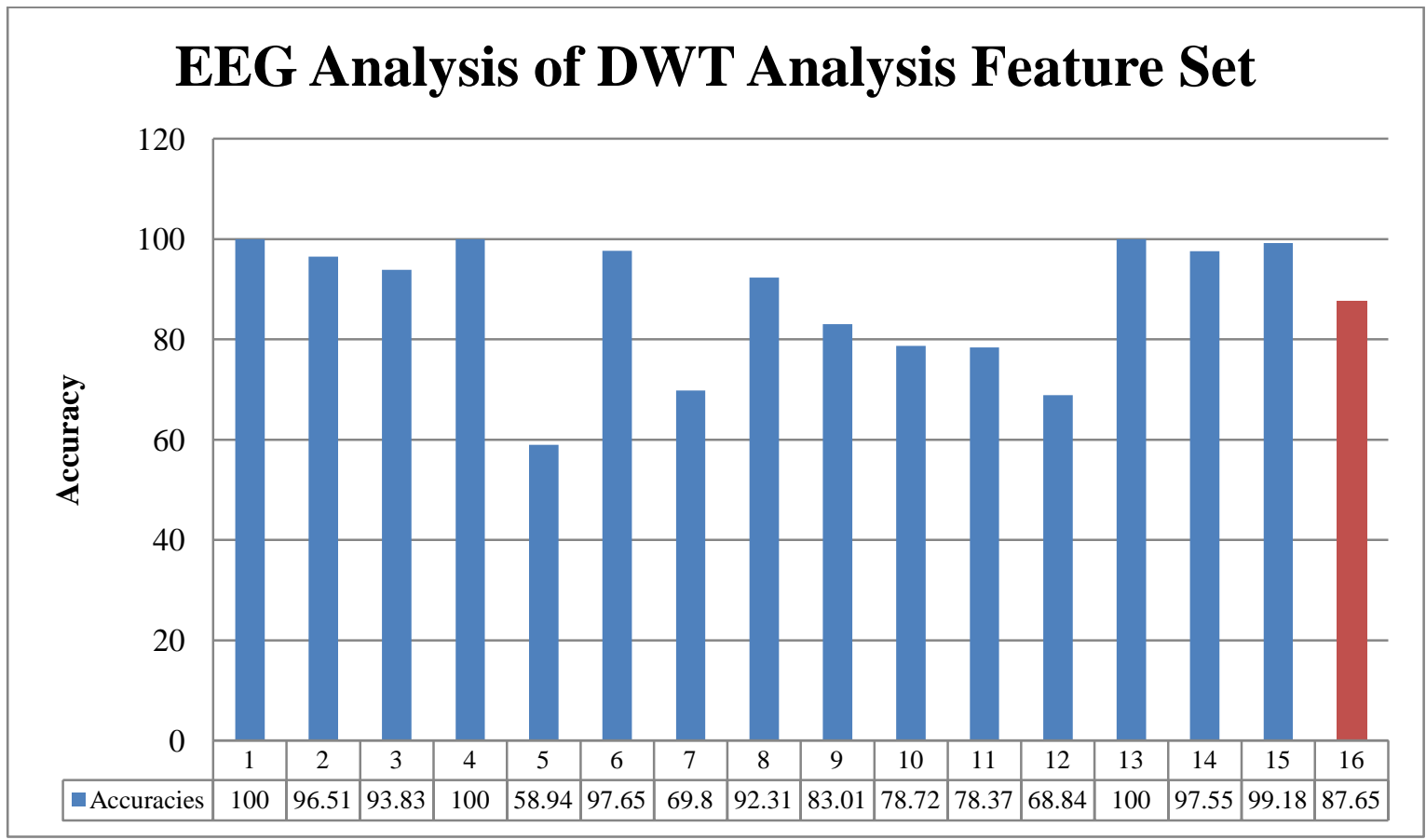

In the table columns 1-15 shows the different accuracies obtained from each by-subject validation for each subject. The mean accuracy of features is $87.64 \%$, which is shown in the table as the red bar on the $17^{\text {nd }}$ column. The mean specificity of the classification over all subjects is $83.78 \%$ and the overall average sensitivity of the classification on feature set of EEG is $90.63 \%$.

With the classification accuracy of ECG analysis (combined features) being $81.11 \%$ and classification accuracy of EEG analysis being $87.64 \%$ it could be said that using ECG for recognizing cognitive attention in subjects is very comparable to that from EEG analysis. However, although these results provide a good direction towards the projects hypothesis, 
the results may not be comparable as equal grounds. The reason being, the number of ECG subjects is just not the same as the EEG subjects. So for a more comprehensive comparison between cases of ECG and EEG, the next section provides subject based ECG to EEG comparisons. Which may be helpful in giving a more clearer picture of how closely the ECG analysis and EEG analysis is comparable. 


\subsection{Comparison of ECG and EEG analysis based on individual subjects}

Due to several circumstantial reasons, the both the ECG and EEG data is not available across the spectrum of subjects on who the experiment was conducted on. None the less, there are 13 specific cases where both ECG and EEG data are available. Using these available cases this section provides a comparison of performance between ECG analysis the corresponding EEG analysis.

\subsubsection{Accuracy Comparison}

Here the ECG data and EEG data of each subject is taken and feature analysis is performed on them separately. After developing a classification model using the feature set of the

remainder of the subjects, the classification statistics of both EEG and ECG is obtained individually. The obtained results are compared side-by-side in the given chart below.

Using the Random Forest Classification method, the accuracies of the classification on the feature set obtained from analysis each subject's ECG and EEG dataset are as follows; 
TABLE 8: ECG AND EEG ANALYSIS CLASSIFICATION ACCURACIES COMPARISON BY SUBJECT

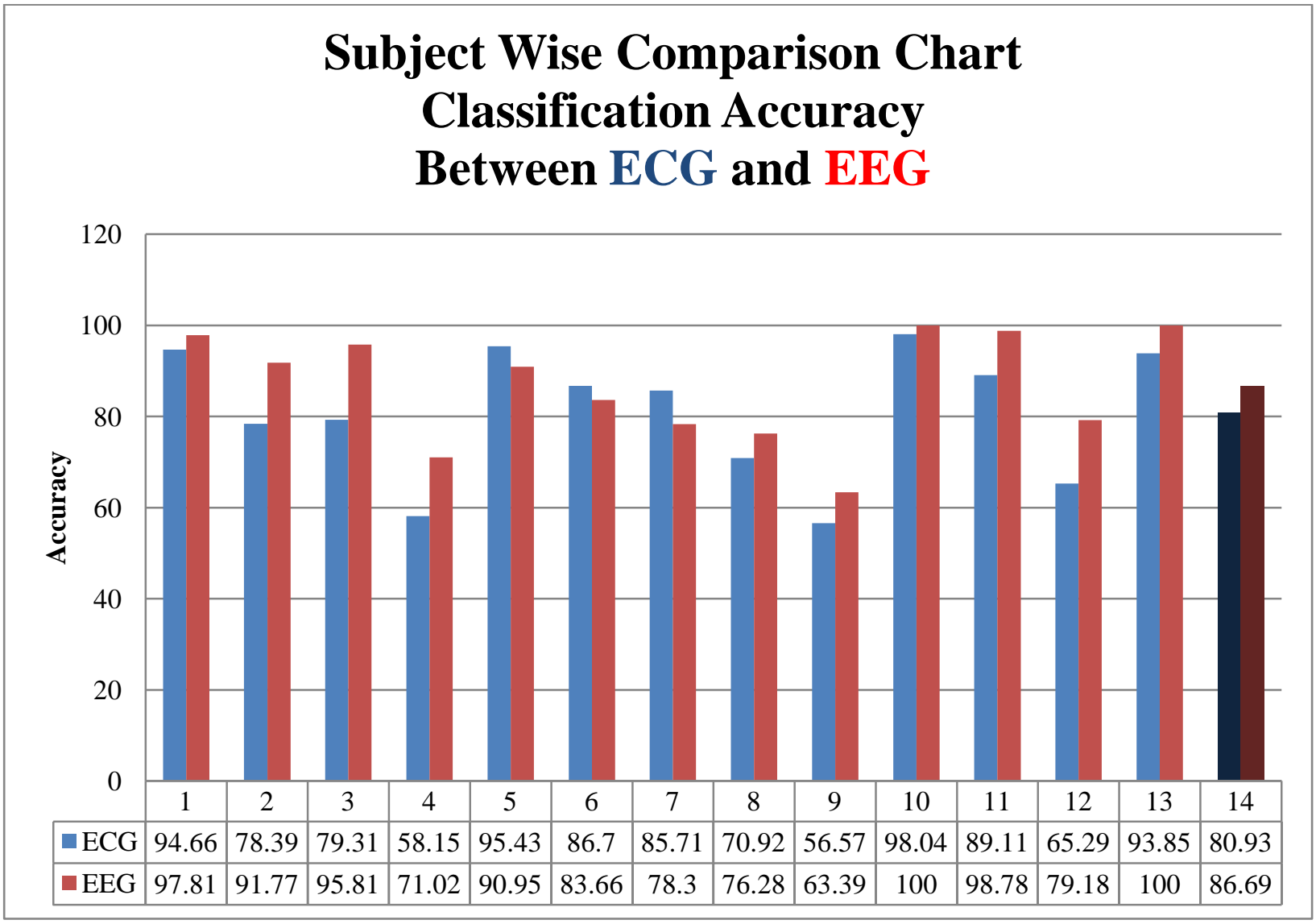

In the table columns 1-13 shows the different accuracies obtained from for ECG (in blue) against EEG (in red) for each by-subject validation of each subject. The mean accuracy classification of ECG is $80.93 \%$, whilst the mean accuracy of the classification of EEG feature set is $86.68 \%$ which is shown in the $14^{\text {nd }}$ column of the table.

For this comparison we can see that in all subject cases ECG results seem to match fairly close as compared to the results obtained from EEG. This shows that analyzing ECG analysis for recognizing cognitive attention is very comparable to that of analyzing EEG. 
This of course holds good when one considers the classification results obtained from EEG for recognizing cognitive attention as the ground truth. It can also be seen that in a few cases the classification results of ECG seems to outperform that of its counterpart EEG analysis. This could possibly be either due to weak EEG signal quality or ECG analysis showing signs of high sensitivity. In any case the comparison of the classification output for each subject ECG against EEG shows a very promising deduction that the project has been able to achieve its goal of using ECG in recognizing cases of attention and noattention in subject especially when in comparison to the assumed ground truth of the analysis output from EEG.

\subsubsection{Preview of intermittent outputs with instance wise comparison}

As described earlier the features are extracted using a windowing method where 10 seconds non-overlapping windowed sections of the original input signal are used. All features extracted from each window is considered as a single instance. This is especially relevant when dealing with the machine learning phase where all the each feature set instance is used to produce a single output of either ' 0 ' for attention and ' 1 ' non cases of lack of attention. So to see how ECG fares in comparison with EEG, for each feature set instance output, a few subject example outputs have been provided below.

Figure 37 and 39 shows the classification output for each feature instance of a particular subject during the section where the subject was asked to view the interesting windows. 
The bars in figure 37(a) figure 39(a) indicate that ECG analysis output for that particular feature set instance shows signs of lack of attention or in other words subject getting bored while viewing the interesting videos, however the EEG analysis during the same instance set does not show any bars in figure 37(b) and figure 39(b). The lack of bars in the EEG analysis shows that there was no lack of attention detected during the viewing of the interesting videos. This shows that ECG misclassified for some of the instance i.e. it shows false positive. This may be due to the high sensitivity nature of the ECG analysis, or just wrongly classified feature instances.

Figure 38 and figure 40 shows the classification output for each feature instance of a particular subject during the section where the subject was asked to view the noninteresting windows. The bars in figure 38(a) and figure 40(a) indicate that ECG analysis output for that particular feature set instance shows signs of lack of attention or in other words subject getting bored while viewing the set of boring videos, Similarly the EEG analysis during the same instance set show bars in figure 38(b) and figure 40(b). The EEG analysis also shows that there was lack of attention was detected during the viewing of the boring videos. 


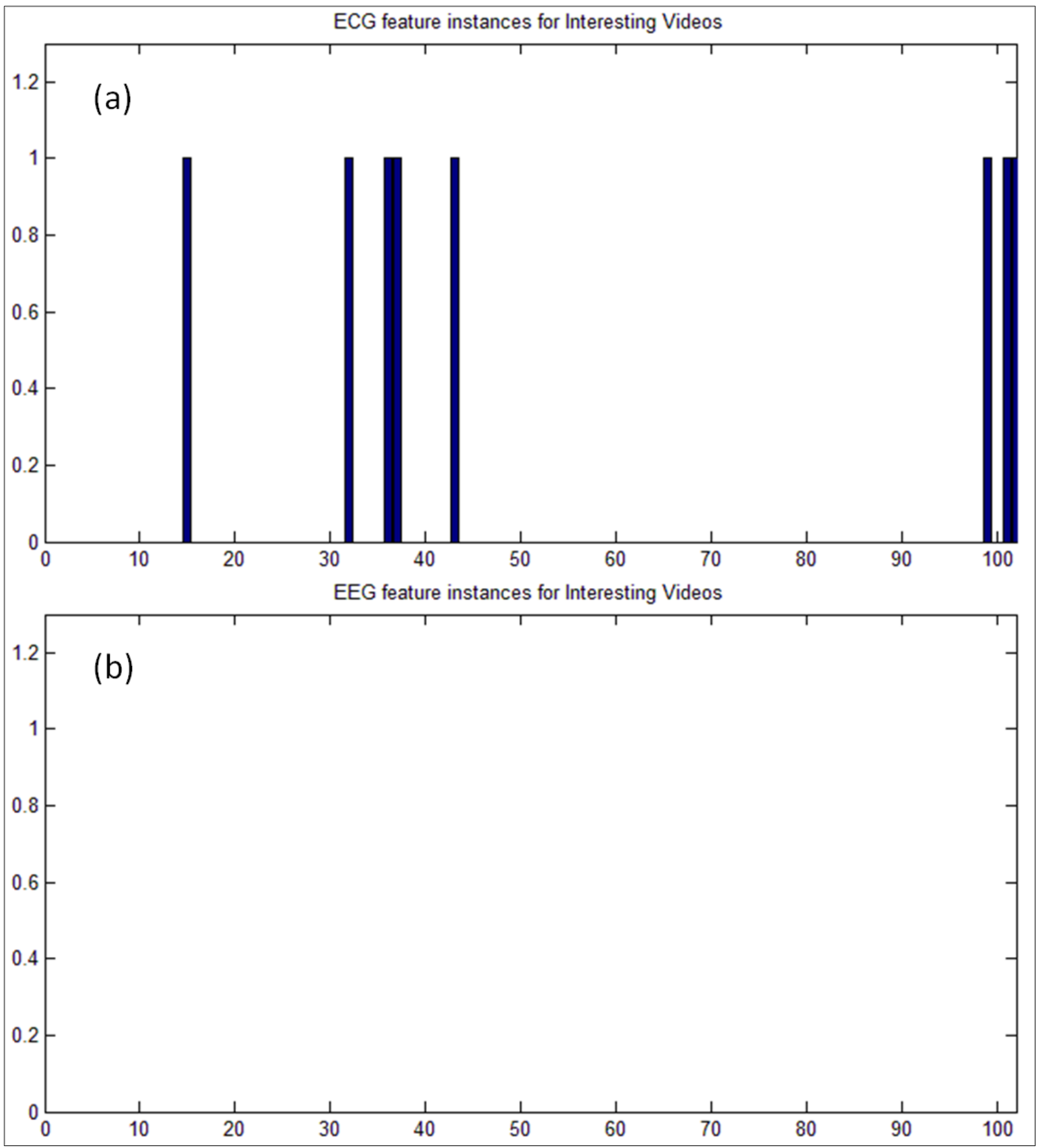

FIGURE 37: CASE 1 INSTANCE BASED COMPARISON OF CLASSIFICATION OUTPUT BETWEEN (A) ECG AND (B) EEG DURINNG INTERESTING VIDEOS SECTION 


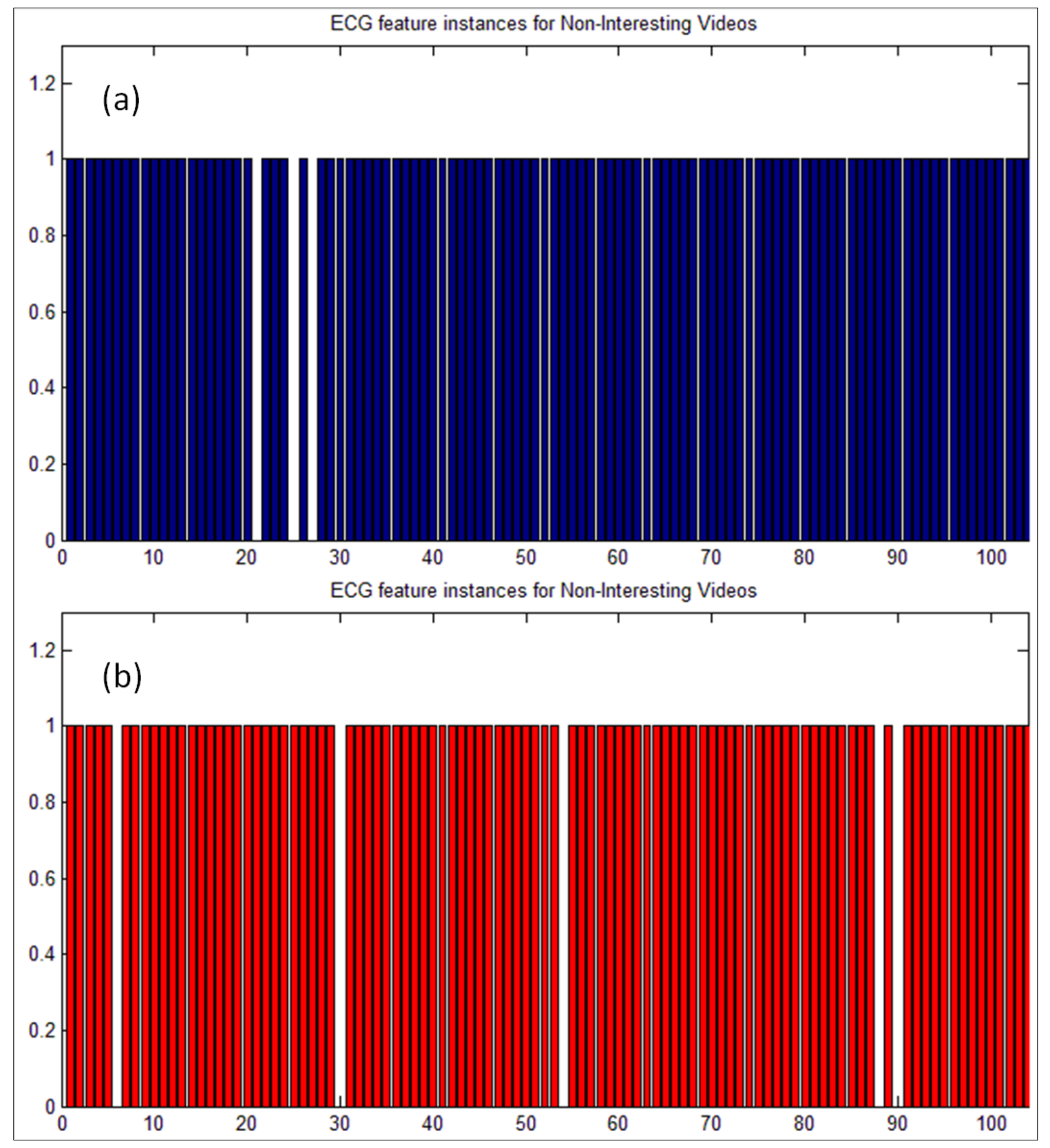

FIGURE 38: CASE 1 INSTANCE BASED COMPARISON OF CLASSIFICATION OUTPUT BETWEEN (A) ECG AND (B) EEG DURINNG BORING VIDEOS SECTION 


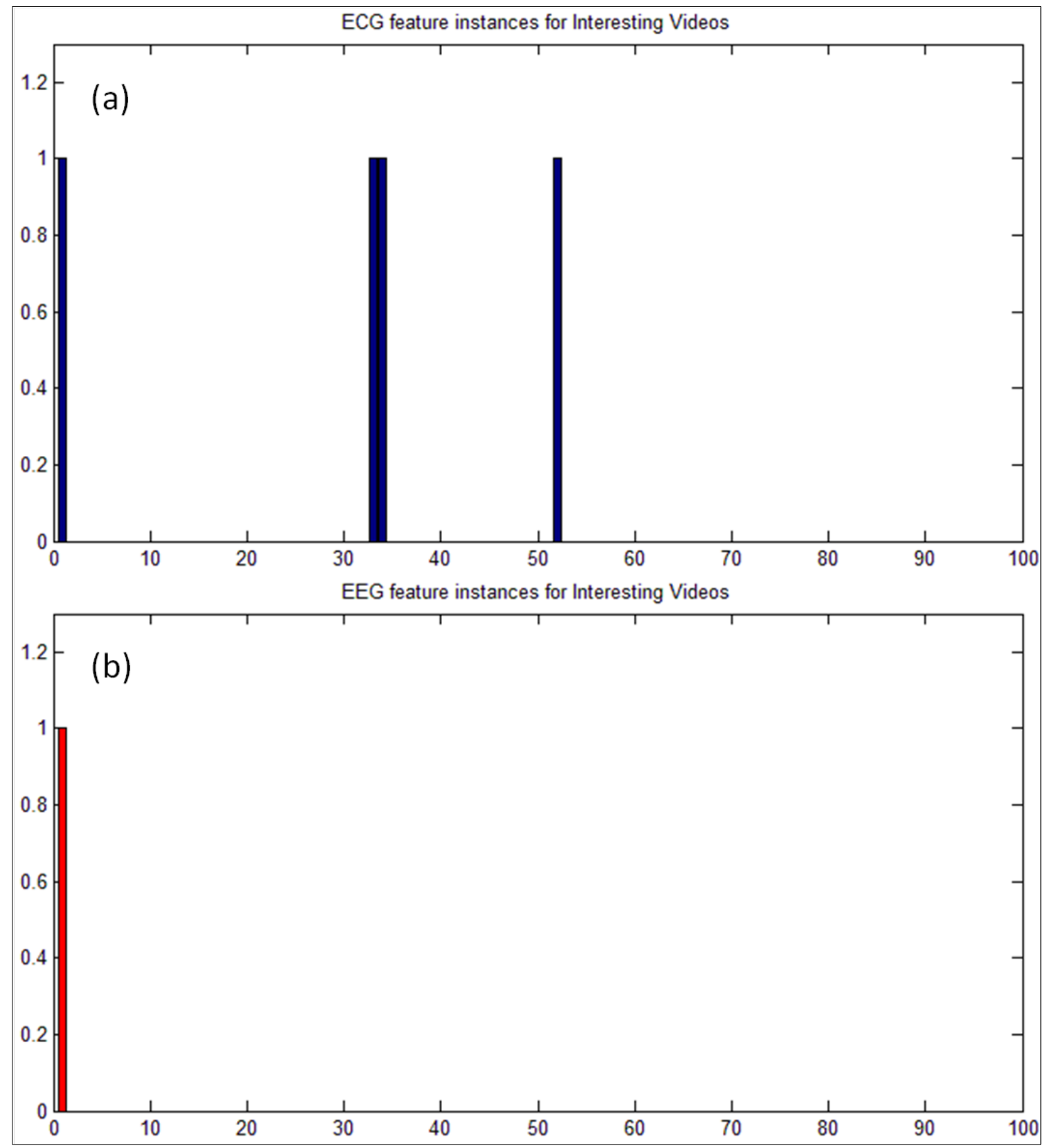

FIGURE 39: CASE 2 INSTANCE BASED COMPARISON OF CLASSIFICATION OUTPUT BETWEEN (A) ECG AND (B) EEG DURINNG INTERESTING VIDEOS SECTION 


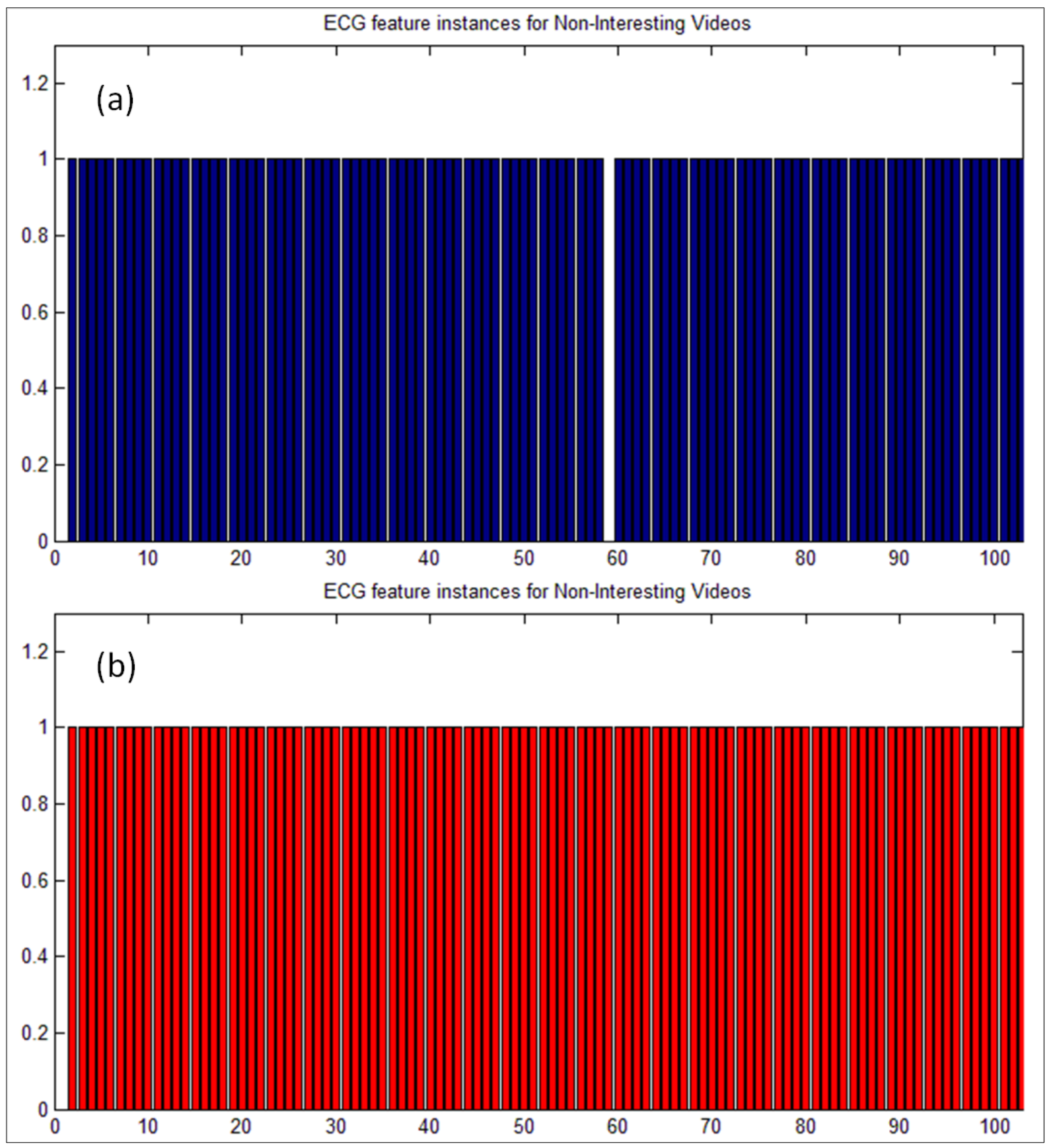

FIGURE 40: CASE 2 INSTANCE BASED COMPARISON OF CLASSIFICATION OUTPUT BETWEEN (A) ECG AND (B) EEG DURING BORING VIDEOS SECTION 


\subsubsection{Table of overall perspectives}

Dwelling further into the subject wise, instance based classification results the following table has been presented. Here overall all confusion analysis output from the classifier for all 13 subject for each of their instances is studies. Based on that the percentage of classification output categories have been computed and presented in the following table.

TABLE 9: TABLE OF OVERALL PERFORMANCE PERSPECTIVE ACCROSS 13 SUBJECT CASES WITH BOTH ECG AND EEG CLASSIFICATION OUTPUTS

\begin{tabular}{|c|c|c|c|c|}
\hline$\%$ & $\begin{array}{c}\text { ECG and } \\
\text { EEG Predict } \\
\text { Correctly }\end{array}$ & $\begin{array}{c}\text { Only ECG } \\
\text { Predicts } \\
\text { Correctly not } \\
\text { EEG }\end{array}$ & $\begin{array}{c}\text { Only EEG } \\
\text { predicts } \\
\text { Correctly not } \\
\text { ECG }\end{array}$ & $\begin{array}{c}\text { Both ECG } \\
\text { and EEG } \\
\text { predict } \\
\text { Incorrectly }\end{array}$ \\
\hline Attention & $78.18 \%$ & $7.09 \%$ & $12.89 \%$ & $1.82 \%$ \\
\hline $\begin{array}{c}\text { No- } \\
\text { Attention }\end{array}$ & $69.29 \%$ & $6.07 \%$ & $15.67 \%$ & $8.95 \%$ \\
\hline
\end{tabular}

The table describes the overall perspective on the performance of the classifier with instance based evaluation. During the section of 'attention', i.e. where the subjects were watching interesting videos, the cases were both ECG and EEG recognized the windowed instances correctly as ‘attention' is about 78\%. For cases where Only ECG recognized it as attention while EEG failed to recognize it as attention for a given instance is only about $7 \%$. About $13 \%$ of the cases exists where instances were recognized correctly as attention 
by EEG while ECG flailed. Finally, there are about $1.82 \%$ of the cases where both ECG and EEG failed to recognize the output instance as attention.

Similarly, the table also provides instance based description for section of 'no-attention', i.e. when the subject was asked to watch the non-interesting or boring set of videos. Here, nearly $69.5 \%$ of the cases of instances were correctly recognized as 'non-interesting' by both ECG and EEG analysis prediction. Furthermore, only about 6\% of the instant cases exist where ECG was able to correctly classify them as non-attention whereas EEG failed to do the same. And in turn, about $15.5 \%$ of the instance cases exist where EEG recognized the instance cases as attentive while ECG misclassified these instances. Finally, the cases where both ECG and EEG failed to classify instance correctly as 'non-interesting' is about $9 \%$.

\subsection{Results developed towards practical application}

Reiterating the goal of this experiment, the aim is to develop an automatic system that monitors human cognitive attention using signals collected from a portable armband such as the ECG. So with that perspective in mind, it is crucial that the system need to be made more designed towards practical application. The experiment as described up to this point shows that ECG analysis using all the described method can be comparable to analysis of EEG for the detection of cognitive attention fluctuation. However it can be recalled that during ECG analysis 396 features are extracted per window of raw signal which is a very 
large number of features. In reality amidst all these extracted features some of these features might not be contributing towards the development of the classification model during machine learning phase. So it can be very helpful if those features that are not good contributors towards the application are identified and eliminated. By doing so, not only is the developed classification model going to be leaner, it also helps in eliminate the computation time required to compute the non-contributing features. Thus making the whole system that much faster and efficient. However after discarding certain features during classification model development, it is important to maintain the same level of classification performance statistics such as accuracy, sensitivity and specificity which were achieved before feature space reduction. For recognizing the usefulness of a feature, info-gain analysis is performed on the entire features set. As mentioned, info-gain is a measure of impurity, which ranks the features based on the quality of separation identified between the labeled cases of interesting and non-interesting for each feature. Based on the computer info-gain indices, the feature can be ranked. 


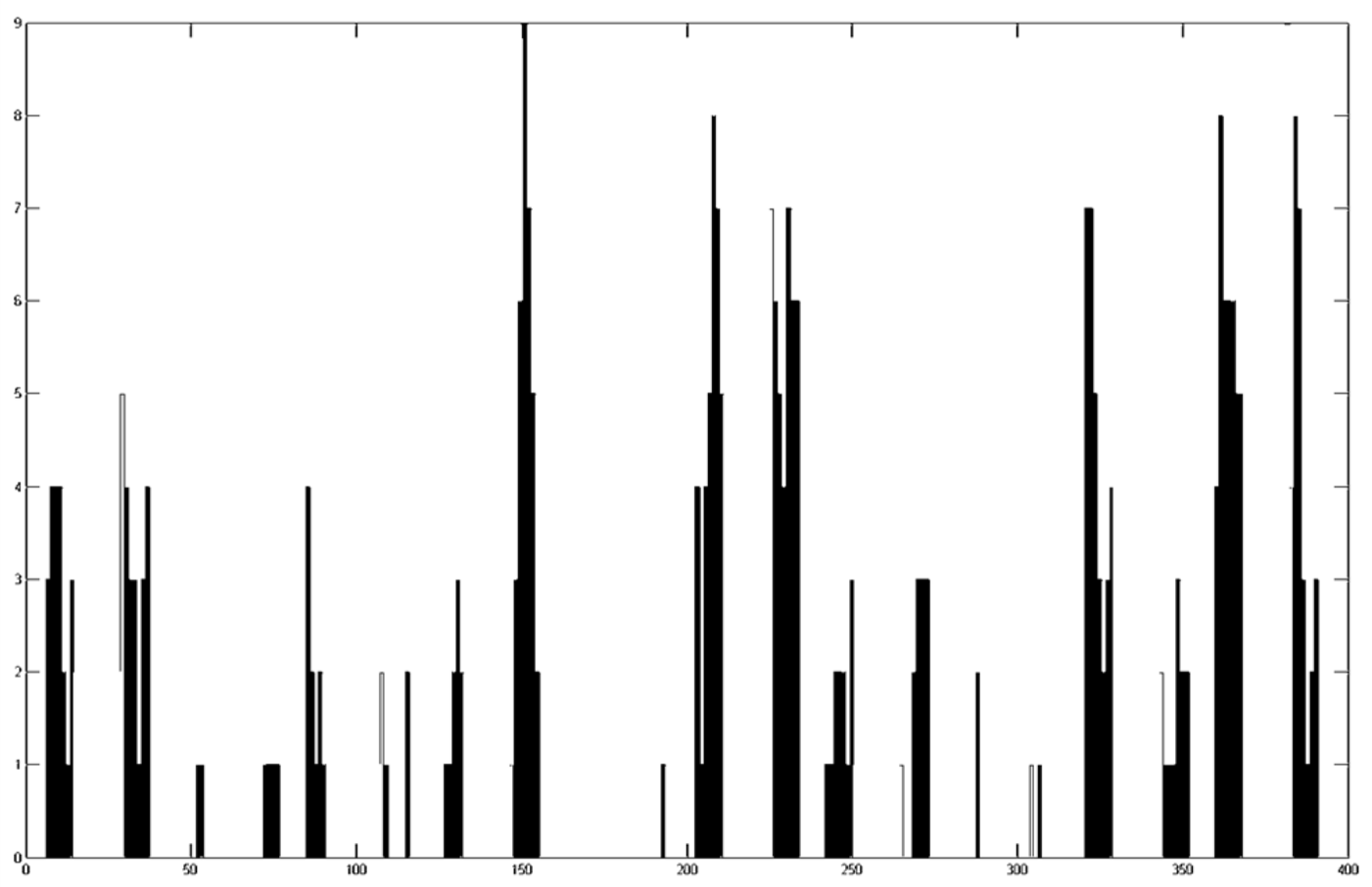

FIGURE 41: FEATURE RANKING, WHERE X-AXIS IS 394 FEATURES NUMBERS AND YAXIS IS NUMBER OF OCCURANCES BASED ON RANK ACCROSS ALL SUBJECTS

Using this ranking information, the top ranked features are then selected and applied for the machine learning stage. To see how many of the top ranked features are required towards the reproduction of the accuracy using all features, a simulation was performed. In this simulation, intermittent step sizes of 10 were for the number of features selected to be used for developing individual classification models. The feature ranking of top 1,11, 21 , $31 \ldots, 301$ and so on were used and the classification output for each step size has been presented in the chart below. 
TABLE 10: MODEL ACCURACIES OBTAINED BY STEP-SIZE RANKED FEATURES

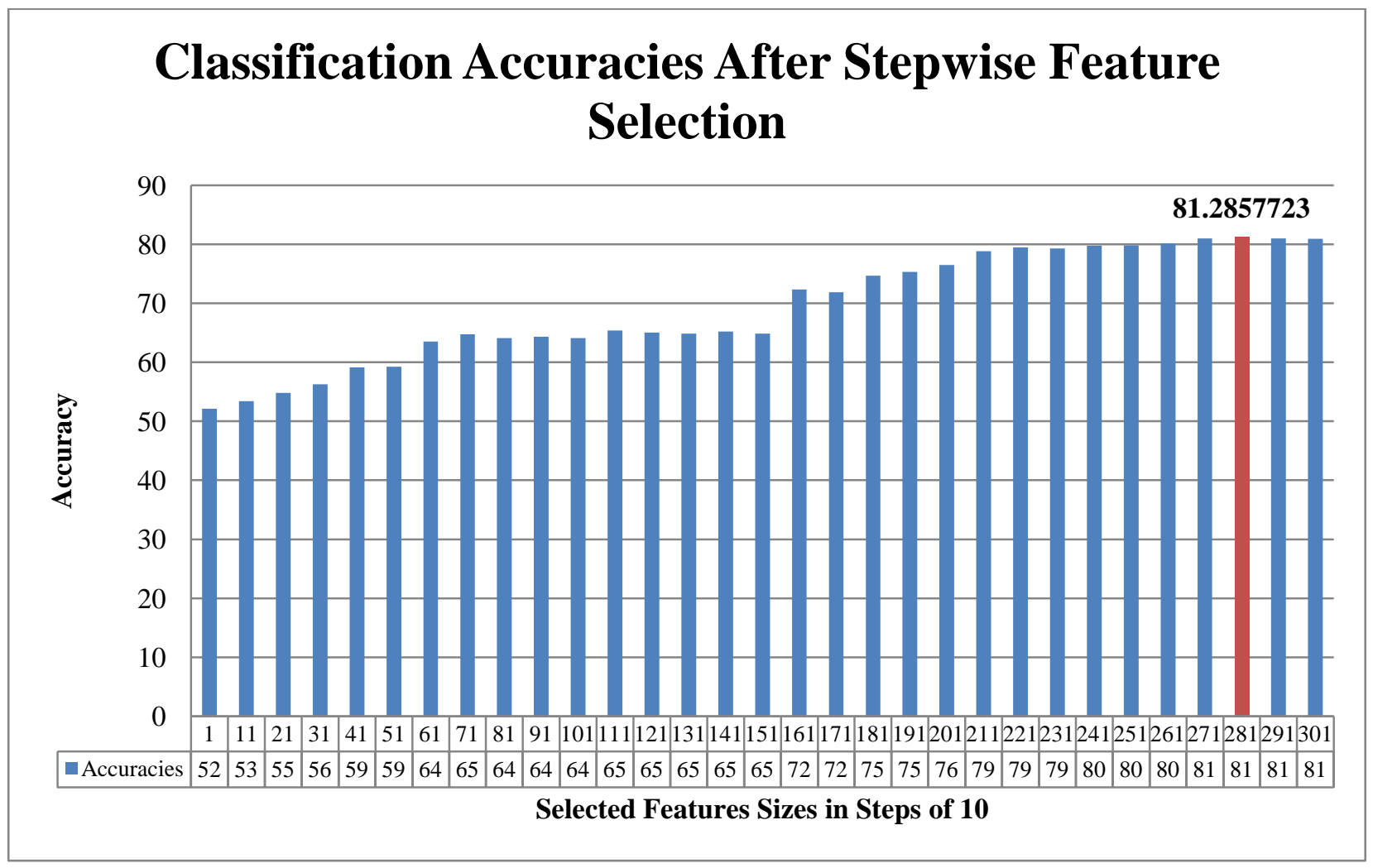

Using top ranked feature sizes of step size 10 from 1 to 301 numbers of features, it can be seen that the classification accuracy starts of at slightly over $50 \%$ with just one feature and then shows an increasing trend as the number of features grows. The classification accuracy peaks at $81.3 \%$ with feature set size of 281 features. Although the graph does not show it, the accuracies do not show any significant change from using feature size between 281 all the way up to 396 (which is the total number of features).

Therefore, it can be seen that by using a feature selection method, it was possible to eliminate nearly a $100+$ features which were found to be not providing any contribution towards the decision making for classification. 
The following table shows the individual accuracies for by subject validation with the 23 available ECG data from subjects.

TABLE 11: CLASSIFICATION ACCURACIES FOR EACH SUBJECT

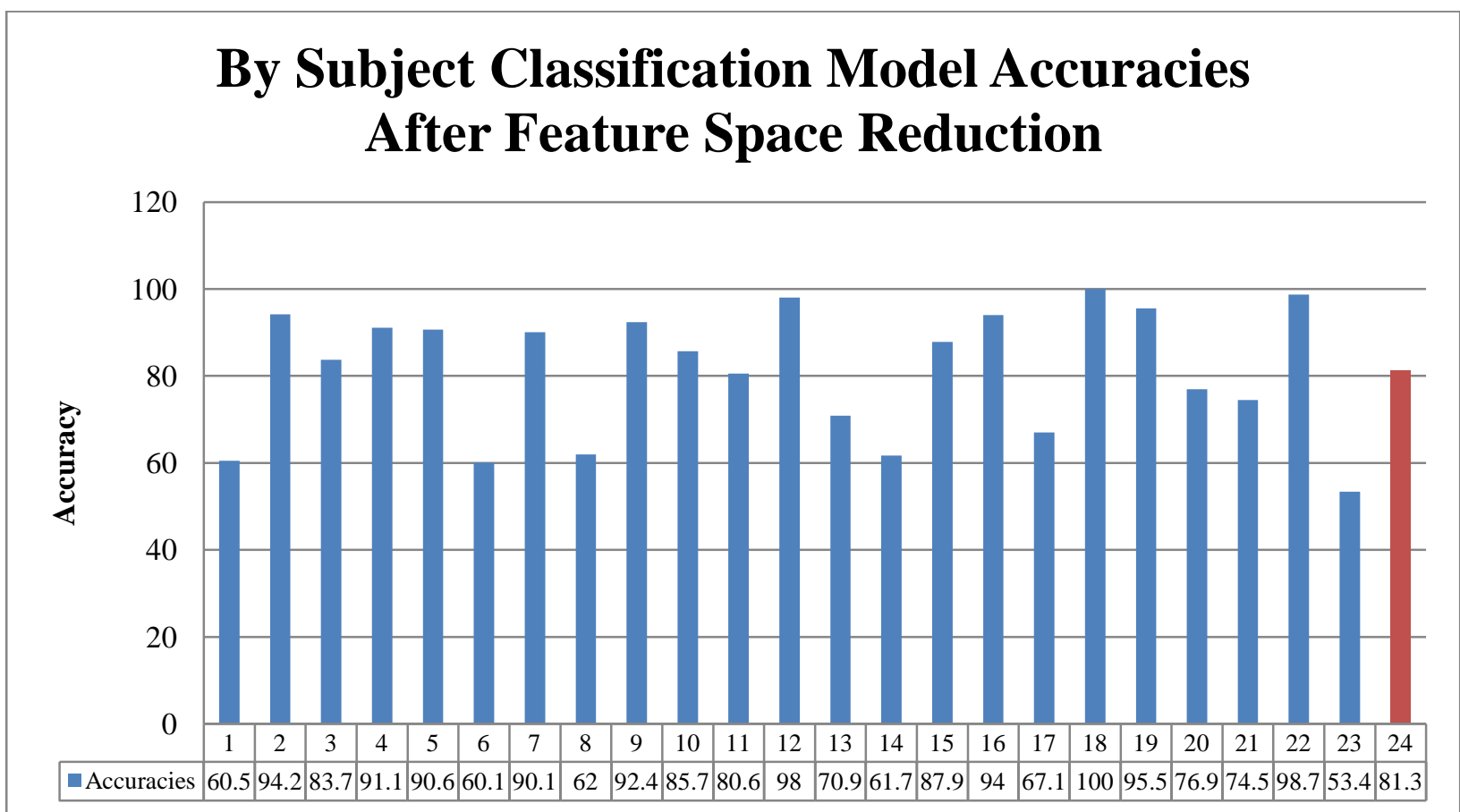

In the table above, columns 1 trough 23 shows the classification accuracy for the ECG data of the 23 subjects. The $24^{\text {th }}$ column shows the mean accuracy across all subjects. 
TABLE 12: CLASSIFICATION OUTPUT COMPARISON TABLE BEFORE AND AFTER FEATURE SPACE REDUCTION

\begin{tabular}{|c|c|c|}
\hline$\%$ & $\begin{array}{c}\text { Before } \\
\text { Feature Space Reduction }\end{array}$ & $\begin{array}{c}\text { After } \\
\text { Feature Space Reduction }\end{array}$ \\
\hline Mean Accuracy & $81.04 \%$ & $81.29 \%$ \\
\hline Mean Sensitivity & $87.74 \%$ & $88.56 \%$ \\
\hline Mean Specificity & $74.77 \%$ & $74.44 \%$ \\
\hline
\end{tabular}

The classification output comparison table shows the classification performance between cases of before feature space reduction and after feature space reduction. It can be seen that both mean accuracy and mean sensitivity of the classification output has seen remained relatively the same both before and after feature space reduction and since fewer features are being used, development of classification models were faster.

To test the performance of the classifier output of the developed model using the top selected 281 features, various outputs were computed by varying the metrics of sensitivity and specificity of the model. This performance measure allows for the plotting of the Receiver Operating Characteristics (ROC) curve, which allows the study of the classification performance as the discriminating threshold is varied. Here the discriminating threshold is fluctuated using the weights input of the random forest towards. By increasing the weights of one class over the other, the sensitivity and specificity of the models performance can be varied. Using the obtained results of true positive rates 
(Sensitivity) and false positive rate (1-specificity) an ROC curve is plotted and the area under the curve is measured. The weight were varied between 1:10 for each class thereby obtaining 10 points on each axes of the ROC plot. Using the intersection of these points on the map, the following ROC curve was obtained.

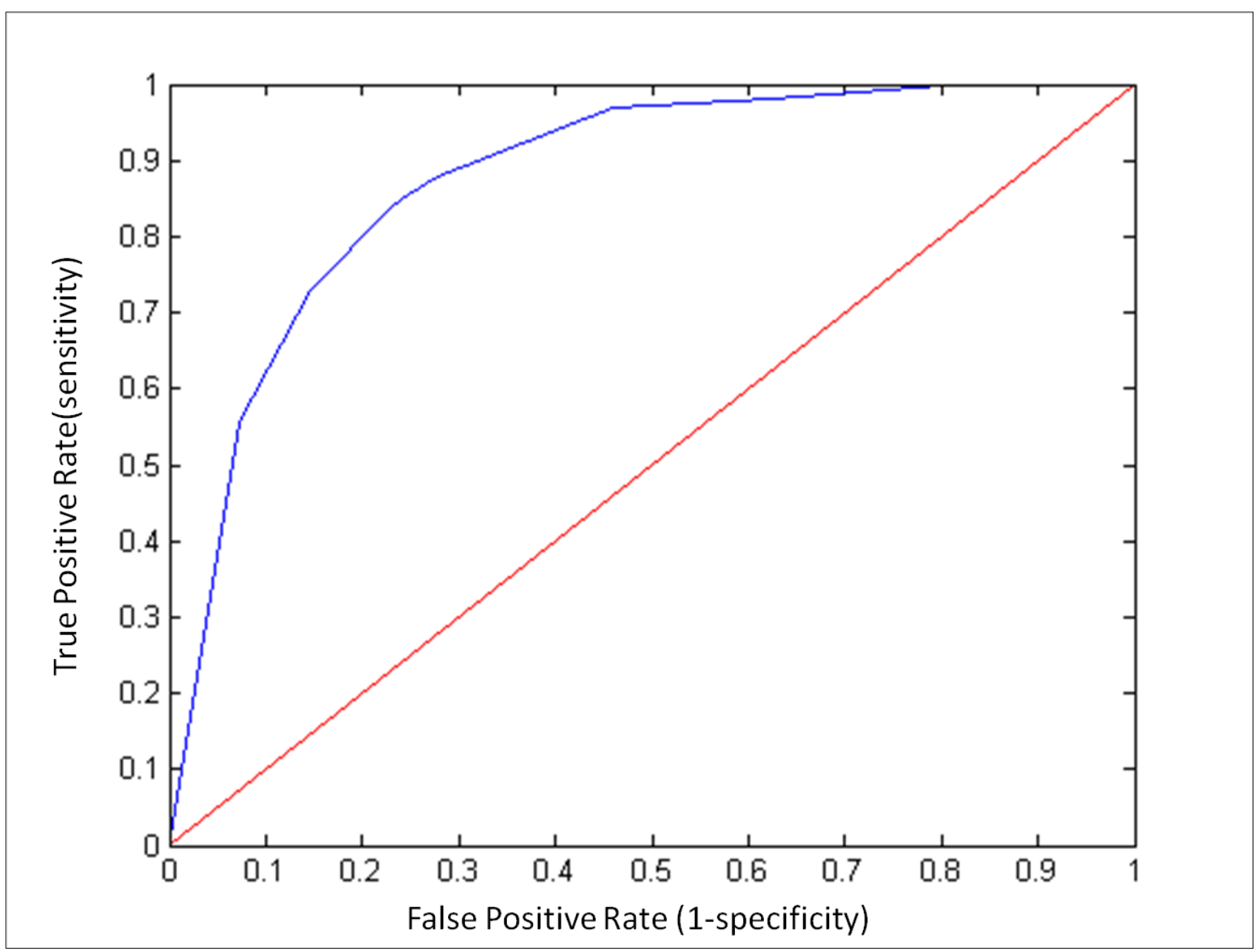

FIGURE 42: RECEIVER OPERATING CHARACTERISTIC OF THE CLASSIFICATION MODEL AFTER FEATURE SELECTION

The Area Under the Curve (AUC) explains how well the model performed. The farther away the blue line is from the red line in the ROC figure, indicates the better performance 
of the classification model. Here the blue line is the plot points of sensitivity and 1specificity values, and the red line is the So the area under the curve is a quantitative way of measuring the separation of the blue to the red line. The calculated area under the curve is $87.7 \%$ indicating that the classification model has shown good performance after feature space reduction.

\subsection{Experimental setup validation using EEG features}

The intention of this section is to use the features extracted from EEG to validate the experimental setup which is used to collect data. For EEG feature space reduction this a novel hybrid nested feature selection algorithms has been developed and described as follows.

With a large feature set of 210 features per subject, reduction of feature space dimension is essential for developing an efficient and accurate classification model. In physiological signals such as the EEG, there exists tremendous inter-personal variability within the features from person to person. Therefore development of a generalized model for classification should factor in such inter-person variability. In this system feature selection is performed using the hybrid model. 


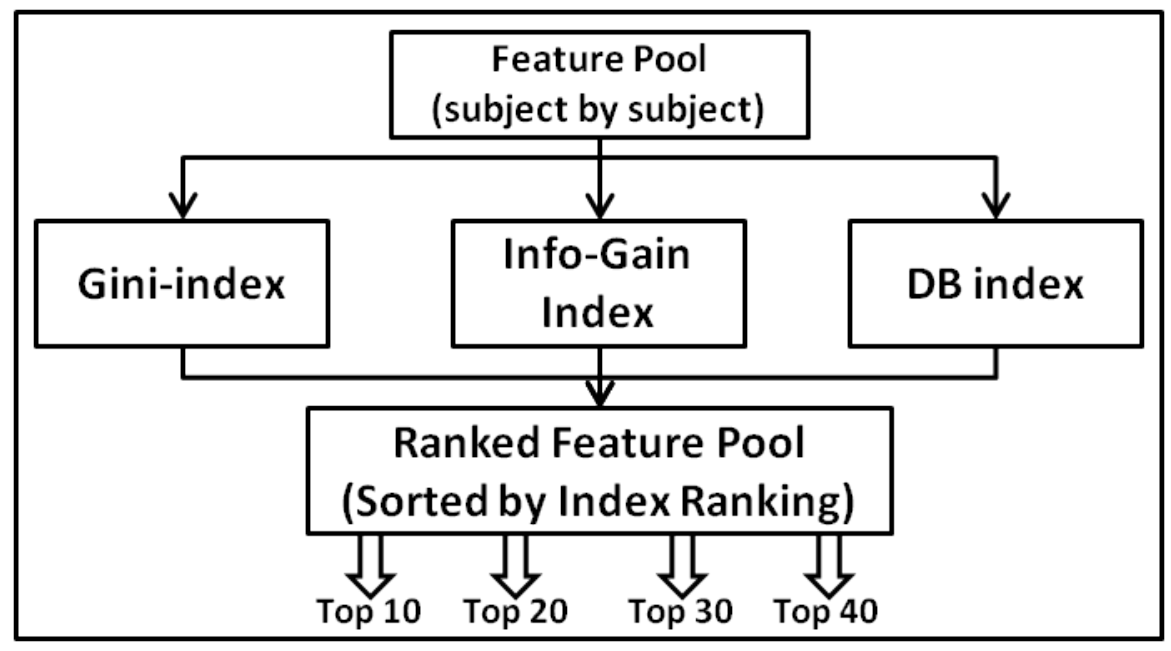

FIGURE 43: HYBRID FEATURE SELECTION ROUTINE

Two measures of impurity are applied as well as one measure of separability. Gini index and entropy (information gain) provide the impurity measures, and DBI measures the separability of each feature's correspondence to the difference in attention. Feature from each subjects feature set is evaluated and ranked using the three feature evaluators separately. The features according to the ranked indices are accumulated in a feature pool. The top ten, twenty, thirty and forty ranked features from this pool are then used for the following calculations where the most popular features with the highest ranks across all feature evaluation methods are selected.

$$
r=h i s t\left\{\begin{array}{c}
R_{\text {gini }} \\
R_{\text {info }} \\
R_{\text {dbi }}
\end{array}\right\}=\left\{\begin{array}{c}
x_{n}(i, j) \rightarrow \text { Gini_Ranks } \\
x_{n}(i, j) \rightarrow \text { InfoGain_Ranks } \\
x_{n}(i, j) \rightarrow \text { DBI_Ranks }
\end{array}\right\}
$$


Where $r$ is the list of number of occurrences of a particular feature in the top $(10,20,30 \& 40)$ ranked feature list. $R_{\text {gini }}, R_{\text {info }}$, and $R_{d b i}$ from the three feature selection methods. $x_{n}(i, j)$ is the feature rank value for each feature of the $n$ by-subject rank list and i,j are the row and column indices of each rank list.

$$
r_{m, n}=\left[\begin{array}{ccc}
r(1,1) & \ldots & r(1, n) \\
& : & \\
r(m, 1) & \ldots & r(m, n)
\end{array}\right](72)
$$

$r_{m, n}$ is the top rank lists where $m$ is the number of features and $n$ is the number of subjects.

$$
a_{m}=\left\{\begin{array}{c}
\sum_{p=1}^{n} r(1, n) \\
\sum_{p=1}^{n} r(2, n) \\
\sum_{p=1}^{n} r(m, n)
\end{array}\right\}
$$

$a_{m}$ is the final list of total ranks computed for each of the top $m$ features and $n$ is the total number of subjects. The final computed list is then sent to the next step of machine learning to find the best feature size $(10,20,30$ or40) for model development and optimization. The advantage of using three different feature ranking methods in this hybrid model is that some features that might not be captured or ranked highly by one method could be ranked higher in the other method/s thereby increasing the diversity of the final feature selections. 


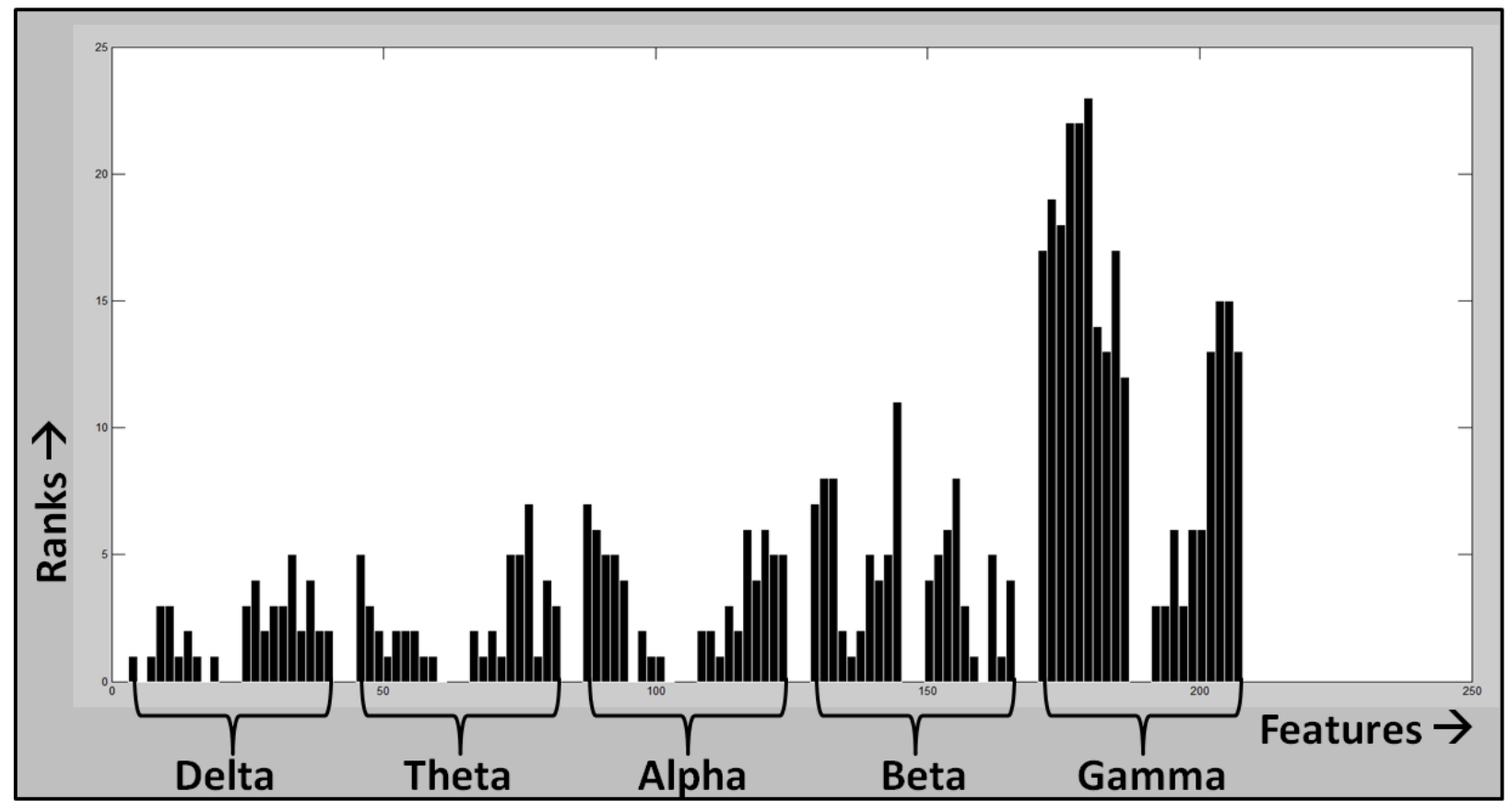

FIGURE 44: FEATURE RANKING BASED ON EACH BAND COMPONENT

From fig 2, it can be noticed that features from the Gamma band have the highest rankings, indicating that Gamma shows the highest change in activity between the two stage of 'attention' and 'non-attention'. This is an important finding since literature review reveals that the in addition to perceptual processing, gamma-band activity accompanies many other cognitive functions such as attention, which validates the success of the feature selection process in extracting and highlighting the essential aspects of the EEG signal which is related to cognitive attention. 


\section{CHAPTER 10 Conclusion and Future Work}

This manuscript proposes a computer assisted attention detection system built on a realtime portable framework. The system is designed to be felicitous towards several different target applications. The functionality of it is based on the analysis of various physiological signals that can be captured wearing a portable armband. In this experiment electroencephalogram (EEG) signals are also captured. The analysis of the EEG signals are primarily to set a benchmark against which the analysis of the physiological features from the armband can be compared. This system as it has been proposed, primarily focuses on the electrocardiogram (ECG) signal and various methods of decomposition are performed on it. The following are the conclusive statements that can be deduced from the systems performance so far;

- It can be seen that to a reasonable level of accuracy the system is able to identify cognitive attention in comparison with that detected by the EEG collected in the same experiment. The focus of this proposal was entirely on ECG alone, and with just this signal it was demonstrated that its classification accuracy was comparable to that of EEG. 
- Amongst the various machine learning methods investigated, 'Random Forest' method seems to perform the best on the combined feature set. Moreover parameter tuning for Random Forest was also successfully demonstrated.

- This study also establishes that ECG alone can be used in analyzing cognitive attention and that the fluctuation of attention has a translated impact on the Cardiac rhythm of an individual.

- One obvious observation that can be made from the results is that prediction from Using Wavelet Transform Decomposition methods alone did not provide very high accuracies, due to the inherent shortcomings of the wavelet transform method. However, by replacing Wavelet Transform with Dual Tree Complex Wavelet transform the accuracy of classification was increased substantially.

- S-transform, Hear rate variability analysis (HRV) and Dual Tree Complex Wavelet transform (Discrete) individually had similar performance statistic, however by combining these methods the accuracy seen to be better than for any one method alone.

- The system by default design was computing and producing an very large feature set which is not always helpful towards overall computation time. Hence A feature pruning method was implemented before developing the final feature set to be used for developing a classification model. Implementing a feature pruning method helped isolate and eliminate those features that seem to be uninformative or hindering the overall classification accuracy of the system. 
- This test bed also proves that such a system can be developed for practical application using a portable framework like that of the armband which negates the need for the requirement of stationary and/or cumbersome physiological data collection machines such as those for collecting EEG.

The study so far has been successful in investigating and establishing the fundamental correlation between ECG and attention in comparison to the already established correlation between EEG and cognition. However, more can be done to improve the overall classification performance of the system in recognizing the fluctuations of cognitive attention levels. Here are some of the future work planned for implementation for the system's classification and prediction performance;

- First and foremost, is the inclusion of other signals from the armband into the analysis scheme. Although initial analysis proved that the Galvanic Skin Response (GSR), and Heat Flux (HF) signals collected as is from the armband did not prove to be informative there is still high potential for these signals to be used towards this Endeavour. So using different sensors to collected these signals can likely improve its performance and this be added onto the overall systems algorithm. This multi-modal analysis is expected to bring down some of the inter-personal variability that is inherent in such physiological based systems.

- More novel features are going to be developed and tried for the feature extraction step after decomposition. Having a more diverse base of features usually provides 
insight into some connate characteristics of the signal which might not be openly evident.

- With hundreds of features being extracted more could be potentially done towards applying and testing newer feature pruning methods. By reducing the number of feature being computed and used for developing the models, the computation time can be greatly reduced and thus making a portable application of this system much more faster and energy efficient.

- A hybrid ensemble super classifier is already being designed and developed for this project which has multiple nested classifiers. The output of all the nested classifiers will be adopted into a novel super classifier scheme which will be used for classification and prediction. The advantage of a multi nested hybrid classifier is that it can reduce interpersonal variability and make the output of the system more uniform across most cases.

- A larger data set will be produced by involving more volunteer subjects into this experiment. A larger data set is expected to provide a more robust classifier model.

- Video based analysis of subject’s facial expression etc., can also be incorporated into the systems as additional features. In practice these video based systems can be applied to relevant applications such as driver monitoring. 


\section{Literature Cited}




\section{References}

[1] R. Descartes, B. de Spinoza, E. S. Haldane, G. R. T. Ross and W. H. White, Rules for the Direction of the Mind: Discourse on the Method; Meditations on First Philosophy; Objections Against the Meditations and Replies; the Geometry. Encyclopaedia Britannica, 1952.

[2] G. Berkeley and C. P. Krauth, A Treatise Concerning the Principles of Human Knowledge. JB Lippincott \& co., 1878.

[3] J. Locke, An Essay Concerning Human Understanding. TE Zell, 1847.

[4] R. D. Caplan, S. Cobb and J. R. P. French, "Job demands and worker health; main effects and occupational differences," Hew Publication (NIOSH), vol. 75, 1975.

[5] T. Cox, A. Griffiths and E. Rial, "Work Related Stress," Occupational Health Psychology, 2010.

[6] M. Frankenhaeuser, B. Nordheden, A. L. Myrsten and B. Post, "Psychophysiological reactions to understimulation and overstimulation," Acta Psychol., vol. 35, pp. 298-308, 1971.

[7] B. Gardell, "Department of Psychology University of Stockholm Technology, Alienation and Mental Health. Summary of a Social Psychological Study of Technology and the Worker," Acta Sociologica, vol. 19, pp. 83-93, 1976.

[8] G. Johansson, G. Aronsson and B. Lindstrom, "Social psychological and neuroendocrine stress reactions in highly mechanised work," Ergonomics, vol. 21, pp. 583599, 1978.

[9] H. Reighard, Aviation Medicine, 1976.

[10] D. F. Dinges and N. B. Kribbs, "Performing while sleepy: Effects of experimentallyinduced sleepiness." 1991.

[11] M. Sallinen, M. Härmä, P. Mutanen, R. Ranta, J. Virkkala and K. Müller, "Sleepwake rhythm in an irregular shift system," J. Sleep Res., vol. 12, pp. 103-112, 2003. 
[12] A. Sharpley, "Impact of daytime sleepiness underrated," The Lancet, vol. 348, pp. 71, 1996.

[13] M. M. Mitler, M. A. Carskadon, C. A. Czeisler, W. C. Dement, D. F. Dinges and R. C. Graeber, "Catastrophes, sleep, and public policy: consensus report," Sleep, vol. 11, pp. 100, 1988.

[14] Anonymous Factors that affect fatigue in heavy truck accidents. National Transportation Safety Board. 1995.

[15] B. C. Tefft, "Asleep at the Wheel: The Prevalence and Impact of Drowsy Driving," 2010 .

[16] P. A. Hancock, "3.1 Active and Passive Fatigue States," Stress, Workload, and Fatigue, pp. 455, 2001.

[17] R. Hockey, Stress and Fatigue in Human Performance. John Wiley \& Sons Inc, 1983.

[18] A. Chaudhuri and P. O. Behan, "Fatigue and basal ganglia," J. Neurol. Sci., vol. 179, pp. 34-42, 2000.

[19] A. Chaudhuri and P. O. Behan, "Fatigue in neurological disorders," The Lancet, vol. 363, pp. 978-988, 2004.

[20] M. M. Lorist, M. Klein, S. Nieuwenhuis, R. Jong, G. Mulder and T. F. Meijman, "Mental fatigue and task control: Planning and preparation," Psychophysiology, vol. 37, pp. 614-625, 2000.

[21] A. F. Sanders, Elements of Human Performance: Reaction Processes and Attention in Human Skill. Lawrence Erlbaum, 1998.

[22] D. van der Linden and P. Eling, "Mental fatigue disturbs local processing more than global processing," Psychol. Res., vol. 70, pp. 395-402, 2006.

[23] P. Bitsios and S. G. Giakoumaki, "Relationship of prepulse inhibition of the startle reflex to attentional and executive mechanisms in man," International Journal of Psychophysiology, vol. 55, pp. 229-241, 2005.

[24] D. L. Filion, K. A. Kelly and E. A. Hazlett, "Behavioral analogies of short lead interval startle inhibition," Startle Modification: Implications for Neuroscience, Cognitive Science, and Clinical Science, pp. 269-283, 1999. 
[25] A. F. Stokes and K. Kite, "1.4 On Grasping and Becoming Emotional," Stress, Workload, and Fatigue, pp. 107, 2001.

[26] D. Tepas and J. Price, "What is stress and what is fatigue," Stress, Workload, and Fatigue.Mahwah, NJ: L.Erlbaum, 2001.

[27] L. E. Bourne and R. A. Yaroush, "Stress and cognition: A cognitive psychological perspective," Unpublished Manuscript, NASA Grant NAG2-1561, 2003.

[28] J. E. Driskell and J. H. Johnston, "Stress exposure training," Making Decisions Under Stress: Implications for Individual and Team Training, pp. 191-217, 1998.

[29] J. E. Driskell and E. Salas, Stress and Human Performance. Lawrence Erlbaum, 1996.

[30] J. E. Driskell, B. Mullen, C. Johnson, S. Hughes and C. L. Batchelor, Development of Quantitative Specifications for Simulating the Stress Environment, 1992.

[31] B. H. Kantowitz and O. Simsek, "2.10 Secondary-Task Measures of Driver Workload," Stress, Workload, and Fatigue, pp. 395, 2001.

[32] D. Gopher and E. Donchin, "Workload: An examination of the concept." 1986.

[33] B. Hilburn and P. G. A. M. Jorna, "2.9 Workload and Air Traffic Control," Stress, Workload, and Fatigue, pp. 384, 2001.

[34] R. Parasuraman and P. A. Hancock, "2.4 Adaptive Control of Mental Workload," Stress, Workload, and Fatigue, pp. 305, 2001.

[35] E. Horvitz and J. Apacible, "Learning and reasoning about interruption," in Proceedings of the 5th International Conference on Multimodal Interfaces, 2003, pp. 2027.

[36] D. S. McCrickard and C. Chewar, "Attuning notification design to user goals and attention costs," Commun ACM, vol. 46, pp. 67-72, 2003.

[37] C. Roda, A. Angehrn, T. Nabeth and L. Razmerita, "Using conversational agents to support the adoption of knowledge sharing practices," Interact Comput, vol. 15, pp. 57-89, 2003.

[38] B. P. Bailey, P. D. Adamczyk, T. Y. Chang and N. A. Chilson, "A framework for specifying and monitoring user tasks," Comput. Hum. Behav., vol. 22, pp. 709-732, 2006. 
[39] R. A. Bolt, "“Put-that-there": Voice and gesture at the graphics interface," in ACM Siggraph Computer Graphics, 1980, pp. 262-270.

[40] M. Weiser, "The computer for the 21st century," Sci. Am., vol. 265, pp. 94-104, 1991.

[41] R. J. K. Jacob, "What you look at is what you get: Eye movement-based interaction techniques," in Proceedings of the SIGCHI Conference on Human Factors in Computing Systems: Empowering People, 1990, pp. 11-18.

[42] J. Nielsen, "Noncommand user interfaces," Commun ACM, vol. 36, pp. 83-99, 1993.

[43] B. Kort, R. Reilly and R. W. Picard, "An affective model of interplay between emotions and learning: Reengineering educational pedagogy-building a learning companion," in Advanced Learning Technologies, 2001. Proceedings. IEEE International Conference on, 2001, pp. 43-46.

[44] J. Raskin, The Humane Interface: New Directions for Designing Interactive Systems. Addison-Wesley Professional, 2000.

[45] G. D. Abowd, "Software engineering issues for ubiquitous computing," in Software Engineering, 1999. Proceedings of the 1999 International Conference on, 1999, pp. 75-84.

[46] E. Horvitz, C. Kadie, T. Paek and D. Hovel, "Models of attention in computing and communication: from principles to applications," Commun ACM, vol. 46, pp. 52-59, 2003.

[47] C. Lisetti, F. Nasoz, C. LeRouge, O. Ozyer and K. Alvarez, "Developing multimodal intelligent affective interfaces for tele-home health care," International Journal of HumanComputer Studies, vol. 59, pp. 245-255, 2003.

[48] N. Oliver, A. Pentland and F. Bérard, "LAFTER: a real-time face and lips tracker with facial expression recognition," Pattern Recognit, vol. 33, pp. 1369-1382, 2000.

[49] N. Ueki, S. Morishima, H. Yamada and H. Harashima, "Expression analysis/synthesis system based on emotion space constructed by multilayered neural network," Syst. Comput. Jpn., vol. 25, pp. 95-107, 1994.

[50] M. Rosenblum, Y. Yacoob and L. S. Davis, "Human expression recognition from motion using a radial basis function network architecture," Neural Networks, IEEE Transactions on, vol. 7, pp. 1121-1138, 1996.

[51] I. A. Essa and A. P. Pentland, "Coding, analysis, interpretation, and recognition of facial expressions," Pattern Analysis and Machine Intelligence, IEEE Transactions on, vol. 19, pp. 757-763, 1997. 
[52] P. S. Della Rocco, T. E. Nesthus, B. Kirwan, M. Rogers and D. Schäfer, "Shift work and air traffic control: Transitioning research results to the workforce," Human Factors Impacts in Air Traffic Management, pp. 243-283, 2005.

[53] L. M. SWANSON, J. ARNEDT, M. R. ROSEKIND, G. BELENKY, T. J. BALKIN and C. DRAKE, "Sleep disorders and work performance: findings from the 2008 National Sleep Foundation Sleep in America poll," J. Sleep Res., vol. 20, pp. 487-494, 2011.

[54] G. Costa, "Evaluation of workload in air traffic controllers," Ergonomics, vol. 36, pp. 1111-1120, 1993.

[55] S. R. Hursh and D. Eddy, "The Fatigue Avoidance Scheduling Tool: Modelling to Minimize the Effects of Fatigue on Cognitive Performance," 2004.

[56] N. Lamond, D. Dawson and G. D. Roach, "Fatigue assessment in the field: validation of a hand-held electronic psychomotor vigilance task," Aviat. Space Environ. Med., vol. 76, pp. 486-489, 2005.

[57] S. Zhai, "What's in the eyes for attentive input," Commun ACM, vol. 46, pp. 34-39, 2003.

[58] S. Hudson, J. Fogarty, C. Atkeson, D. Avrahami, J. Forlizzi, S. Kiesler, J. Lee and J. Yang, "Predicting human interruptibility with sensors: A wizard of oz feasibility study," in Proceedings of the SIGCHI Conference on Human Factors in Computing Systems, 2003, pp. 257-264.

[59] D. Chen and R. Vertegaal, "Using mental load for managing interruptions in physiologically attentive user interfaces," in CHI'04 Extended Abstracts on Human Factors in Computing Systems, 2004, pp. 1513-1516.

[60] H. Heinrich, H. Gevensleben and U. Strehl, "Annotation: Neurofeedback-train your brain to train behaviour," Journal of Child Psychology and Psychiatry, vol. 48, pp. 3-16, 2007.

[61] M. Holtmann and C. Stadler, "Electroencephalographic biofeedback for the treatment of attention-deficit hyperactivity disorder in childhood and adolescence," Expert Review of Neurotherapeutics, vol. 6, pp. 533-540, 2006.

[62] P. M. Kidd, "Attention deficit/hyperactivity disorder (ADHD) in children: rationale for its integrative management," Alternative Medicine Review, vol. 5, pp. 402-428, 2000.

[63] P. S. Jensen, "Current concepts and controversies in the diagnosis and treatment of attention deficit hyperactivity disorder," Curr. Psychiatry Rep., vol. 2, pp. 102-109, 2000. 
[64] C. B. Liden, M. Wolowicz, J. Stivoric, A. Teller, C. Kasabach, S. Vishnubhatla, R. Pelletier, J. Farringdon and S. Boehmke, "Characterization and Implications of the Sensors Incorporated into the SenseWear ${ }^{\mathrm{TM}}$ Armband for Energy Expenditure and Activity Detection," Bodymedia Inc.White Papers: 1, vol. 7, 2002.

[65] D. Andre, R. Pelletier, J. Farringdon, S. Safier, W. Talbott, R. Stone, N. Vyas, J. Trimble, D. Wolf and S. Vishnubhatla, "The Development of the SenseWear ${ }^{\circledR}$ armband, a Revolutionary Energy Assessment Device to Assess Physical Activity and Lifestyle," BodyMedia Inc, 2006.

[66] Ahmad, Homer and Wang, "Accuracy and utility of multi-sensor armband ECG signal compared to holder monitoring," in Presented at New Arrhythmia Technologies Retreat, Chicago, IL. USA., 2004, .

[67] S. Karpagachelvi, M. Arthanari and M. Sivakumar, "ECG Feature extraction techniques-A survey approach," Arxiv Preprint arXiv:1005.0957, 2010.

[68] J. P. Martínez, R. Almeida, S. Olmos, A. P. Rocha and P. Laguna, "A wavelet-based ECG delineator: evaluation on standard databases," Biomedical Engineering, IEEE Transactions on, vol. 51, pp. 570-581, 2004.

[69] G. K. Prasad and J. Sahambi, "Classification of ECG arrhythmias using multiresolution analysis and neural networks," in TENCON 2003. Conference on Convergent Technologies for Asia-Pacific Region, 2003, pp. 227-231 Vol. 1.

[70] C. Li, C. Zheng and C. Tai, "Detection of ECG characteristic points using wavelet transforms," Biomedical Engineering, IEEE Transactions on, vol. 42, pp. 21-28, 1995.

[71] C. Saritha, V. Sukanya and Y. N. Murthy, "ECG signal analysis using wavelet transforms," Bulg. J. Phys., vol. 35, pp. 68-77, 2008.

[72] S. J. Orfanidis, Introduction to Signal Processing. Prentice-Hall, Inc., 1995.

[73] M. A. Awal, S. S. Mostafa and M. Ahmad, "Performance Analysis of Savitzky-Golay Smoothing Filter Using ECG Signal," 2011.

[74] G. M. Friesen, T. C. Jannett, M. A. Jadallah, S. L. Yates, S. R. Quint and H. T. Nagle, "A comparison of the noise sensitivity of nine QRS detection algorithms," Biomedical Engineering, IEEE Transactions on, vol. 37, pp. 85-98, 1990.

[75] O. Pahlm and L. Sörnmo, "Software QRS detection in ambulatory monitoring-a review," Medical and Biological Engineering and Computing, vol. 22, pp. 289-297, 1984. 
[76] B. U. Kohler, C. Hennig and R. Orglmeister, "The principles of software QRS detection," Engineering in Medicine and Biology Magazine, IEEE, vol. 21, pp. 42-57, 2002.

[77] P. S. Addison, "Wavelet transforms and the ECG: a review," Physiol. Meas., vol. 26, pp. R155, 2005.

[78] M. Bahoura, M. Hassani and M. Hubin, "DSP implementation of wavelet transform for real time ECG wave forms detection and heart rate analysis," Comput. Methods Programs Biomed., vol. 52, pp. 35-44, 1997.

[79] I. Romero Legarreta, P. Addison, N. Grubb, G. Clegg, C. Robertson, K. Fox and J. Watson, "R-wave detection using continuous wavelet modulus maxima," in Computers in Cardiology, 2003, 2003, pp. 565-568.

[80] S. Mahmoodabadi, A. Ahmadian, M. Abolhasani, M. Eslami and J. Bidgoli, "ECG feature extraction based on multiresolution wavelet transform," in Engineering in Medicine and Biology Society, 2005. IEEE-EMBS 2005. 27th Annual International Conference of the, 2006, pp. 3902-3905.

[81] R. Sunkaria, S. Saxena, V. Kumar and A. Singhal, "Wavelet based R-peak detection for heart rate variability studies," J. Med. Eng. Technol., vol. 33, pp. 108-115, 2010.

[82] Q. Xue, Y. H. Hu and W. J. Tompkins, "Neural-network-based adaptive matched filtering for QRS detection," Biomedical Engineering, IEEE Transactions on, vol. 39, pp. 317-329, 1992.

[83] G. Vijaya, V. Kumar and H. Verma, "ANN-based QRS-complex analysis of ECG," J. Med. Eng. Technol., vol. 22, pp. 160-167, 1998.

[84] P. Trahanias, "An approach to QRS complex detection using mathematical morphology," Biomedical Engineering, IEEE Transactions on, vol. 40, pp. 201-205, 1993.

[85] Y. Sun, K. Chan and S. Krishnan, "Characteristic wave detection in ECG signal using morphological transform," BMC Cardiovascular Disorders, vol. 5, pp. 28, 2005.

[86] Y. Sun, K. L. Chan and S. M. Krishnan, "ECG signal conditioning by morphological filtering," Comput. Biol. Med., vol. 32, pp. 465-479, 2002.

[87] A. A. R. Bsoul, S. Y. Ji, K. Ward and K. Najarian, "Detection of P, QRS, and T components of ECG using wavelet transformation," in International Conference on Complex Medical Engineering, 2009, . 
[88] H. D. Critchley, "Psychophysiology of neural, cognitive and affective integration: fMRI and autonomic indicants," International Journal of Psychophysiology, vol. 73, pp. 88-94, 2009.

[89] K. Hugdahl, "Cognitive influences on human autonomic nervous system function," Curr. Opin. Neurobiol., vol. 6, pp. 252-258, 1996.

[90] J. R. Jennings, "Is it important that the mind is in a body? Inhibition and the heart," Psychophysiology, vol. 29, pp. 369-383, 1992.

[91] J. R. Jennings, M. W. Van der Molen, K. Brock and R. J. M. Somsen, "Response Inhibition Initiates Cardiac Deceleration: Evidence from a Sensory-Motor Compatibility Paradigm," Psychophysiology, vol. 28, pp. 72-85, 1991.

[92] M. W. Molen, D. I. Boomsma, J. R. Jennings and R. T. Nieuwboer, "Does the heart know what the eye sees? A cardiac/pupillometric analysis of motor preparation and response execution," Psychophysiology, vol. 26, pp. 70-80, 1989.

[93] G. Bohlin, "„\& Kjellberg, A.(1979). Orienting activity in two-stimulus paradigms as reflected in heart rate," The Orienting Reflex in Humans, pp. 169-197, .

[94] P. Goupillaud, A. Grossmann and J. Morlet, "Cycle-octave and related transforms in seismic signal analysis," Geoexploration, vol. 23, pp. 85-102, 1984.

[95] M. Khalil and J. Duchêne, "Uterine EMG analysis: a dynamic approach for change detection and classification," Biomedical Engineering, IEEE Transactions on, vol. 47, pp. 748-756, 2000.

[96] A. Petrosian, D. Prokhorov, R. Homan, R. Dasheiff and D. Wunsch, "Recurrent neural network based prediction of epileptic seizures in intra-and extracranial EEG," Neurocomputing, vol. 30, pp. 201-218, 2000.

[97] L. J. Hadjileontiadis and S. M. Panas, "Separation of discontinuous adventitious sounds from vesicular sounds using a wavelet-based filter," Biomedical Engineering, IEEE Transactions on, vol. 44, pp. 1269-1281, 1997.

[98] P. Dupuis and C. Eugene, "Combined detection of respiratory and cardiac rhythm disorders by high-resolution differential cuff pressure measurement," Instrumentation and Measurement, IEEE Transactions on, vol. 49, pp. 498-502, 2000.

[99] A. Marrone, A. Polosa, G. Scioscia, S. Stramaglia and A. Zenzola, "Multiscale analysis of blood pressure signals," Physical Review E, vol. 60, pp. 1088, 1999. 
[100] A. Arneodo, Y. d'Aubenton-Carafa, B. Audit, E. Bacry, J. Muzy and C. Thermes, "Nucleotide composition effects on the long-range correlations in human genes," EUROPEAN PHYSICAL JOURNAL B CONDENSED MATTER, vol. 1, pp. 259-263, 1998.

[101] B. Castro, D. Kogan and A. Geva, "ECG feature extraction using optimal mother wavelet," in Electrical and ELectronic Engineers in Israel, 2000. the 21st IEEE Convention of the, 2000, pp. 346-350.

[102] K. Najarian and R. Splinter, Biomedical Signal and Image Processing. CRC press, 2006.

[103] I. Daubechies, Ten Lectures on Wavelets. Society for Industrial Mathematics, 1992.

[104] S. Chouakri, F. Bereksi-Reguig, S. Ahmaidi and O. Fokapu, "Wavelet denoising of the electrocardiogram signal based on the corrupted noise estimation," in Computers in Cardiology, 2005, 2005, pp. 1021-1024.

[105] S. G. Mallat, "A theory for multiresolution signal decomposition: The wavelet representation," Pattern Analysis and Machine Intelligence, IEEE Transactions on, vol. 11, pp. 674-693, 1989.

[106] M. Portnoff, "Time-frequency representation of digital signals and systems based on short-time Fourier analysis," Acoustics, Speech and Signal Processing, IEEE Transactions on, vol. 28, pp. 55-69, 1980.

[107] D. Gabor, "Theory of communication. Part 1: The analysis of information," Electrical Engineers-Part III: Radio and Communication Engineering, Journal of the Institution of, vol. 93, pp. 429-441, 1946.

[108] P. Bloomfield, Fourier Analysis of Time Series: An Introduction. Wiley-Interscience, 2004.

[109] L. Cohen, "Time-frequency distributions-a review," Proc IEEE, vol. 77, pp. 941981, 1989.

[110] Y. Zhao, L. E. Atlas and R. J. Marks, "The use of cone-shaped kernels for generalized time-frequency representations of nonstationary signals," Acoustics, Speech and Signal Processing, IEEE Transactions on, vol. 38, pp. 1084-1091, 1990.

[111] H. I. Choi and W. J. Williams, "Improved time-frequency representation of multicomponent signals using exponential kernels," Acoustics, Speech and Signal Processing, IEEE Transactions on, vol. 37, pp. 862-871, 1989. 
[112] F. Hlawatsch and G. Boudreaux-Bartels, "Linear and quadratic time-frequency signal representations," Signal Processing Magazine, IEEE, vol. 9, pp. 21-67, 1992.

[113] R. Stockwell, L. Mansinha and R. Lowe, "Localization of the complex spectrum: the S transform," Signal Processing, IEEE Transactions on, vol. 44, pp. 998-1001, 1996.

[114] J. L. Starck and F. Murtagh, "Astronomical image and signal processing: looking at noise, information and scale," Signal Processing Magazine, IEEE, vol. 18, pp. 30-40, 2001.

[115] M. Vetterli, "Wavelets, approximation, and compression," Signal Processing Magazine, IEEE, vol. 18, pp. 59-73, 2001.

[116] H. Choi, J. Romberg, R. Baraniuk and N. Kingsbury, "Hidden markov tree modeling of complex wavelet transforms," in Acoustics, Speech, and Signal Processing, 2000.

ICASSP'00. Proceedings. 2000 IEEE International Conference on, 2000, pp. 133-136 vol. 1.

[117] N. G. Kingsbury, "The dual-tree complex wavelet transform: A new technique for shift invariance and directional filters," in Proc. 8th IEEE DSP Workshop, 1998, .

[118] J. Romberg, H. Choi, R. Baraniuk and N. Kingbury, "Multiscale classification using complex wavelets and hidden markov tree models," in Image Processing, 2000.

Proceedings. 2000 International Conference on, 2000, pp. 371-374 vol. 2.

[119] I. W. Selesnick, R. G. Baraniuk and N. C. Kingsbury, "The dual-tree complex wavelet transform," Signal Processing Magazine, IEEE, vol. 22, pp. 123-151, 2005.

[120] N. Kingsbury, "Complex wavelets for shift invariant analysis and filtering of signals," Applied and Computational Harmonic Analysis, vol. 10, pp. 234-253, 2001.

[121] A. Babloyantz, J. Salazar and C. Nicolis, "Evidence of chaotic dynamics of brain activity during the sleep cycle," Physics Letters A, vol. 111, pp. 152-156, 1985.

[122] K. Natarajan, R. Acharya, F. Alias, T. Tiboleng and S. Puthusserypady, "Nonlinear analysis of EEG signals at different mental states," BioMedical Engineering OnLine, vol. 3, pp. 7, 2004.

[123] A. R. Clarke, R. J. Barry, R. McCarthy and M. Selikowitz, "EEG analysis in attention-deficit/hyperactivity disorder: a comparative study of two subtypes," Psychiatry Res., vol. 81, pp. 19-29, 1998. 
[124] J. F. Lubar, "Discourse on the development of EEG diagnostics and biofeedback for attention-deficit/hyperactivity disorders," Appl. Psychophysiol. Biofeedback, vol. 16, pp. 201-225, 1991.

[125] T. P. Tinius and K. A. Tinius, "Changes after EEG biofeedback and cognitive retraining in adults with mild traumatic brain injury and attention deficit hyperactivity disorder," Journal of Neurotherapy, vol. 4, pp. 27-44, 2000.

[126] P. Fries, "A mechanism for cognitive dynamics: neuronal communication through neuronal coherence," Trends Cogn. Sci. (Regul. Ed. ), vol. 9, pp. 474-480, 2005.

[127] J. Fell and N. Axmacher, "The role of phase synchronization in memory processes," Nature Reviews Neuroscience, vol. 12, pp. 105-118, 2011.

[128] A. Schnitzler and J. Gross, "Normal and pathological oscillatory communication in the brain," Nature Reviews Neuroscience, vol. 6, pp. 285-296, 2005.

[129] J. B. Ochoa, "Eeg signal classification for brain computer interface applications," Ecole Polytechnique Federale De Lausanne, 2002.

[130] S. Butterworth, "On the theory of filter amplifiers," Wireless Engineer, vol. 7, pp. 536-541, 1930.

[131] E. Başar, C. Başar-Eroğlu, S. Karakaş and M. Schürmann, "Brain oscillations in perception and memory," International Journal of Psychophysiology, vol. 35, pp. 95-124, 2000 .

[132] E. Başar, EEG-Brain Dynamics: Relation between EEG and Brain Evoked Potentials. Elsevier-North-Holland Biomedical Press, 1980.

[133] V. J. Samar, A. Bopardikar, R. Rao and K. Swartz, "Wavelet analysis of neuroelectric waveforms: a conceptual tutorial," Brain Lang., vol. 66, pp. 7-60, 1999.

[134] V. J. Samar, A. Bopardikar, R. Rao and K. Swartz, "Wavelet analysis of neuroelectric waveforms: a conceptual tutorial," Brain Lang., vol. 66, pp. 7-60, 1999.

[135] E. Bartnik, K. Blinowska and P. Durka, "Single evoked potential reconstruction by means of wavelet transform," Biol. Cybern., vol. 67, pp. 175-181, 1992.

[136] O. Bertrand, J. Bohorquez and J. Pernier, "Time-frequency digital filtering based on an invertible wavelet transform: an application to evoked potentials," Biomedical Engineering, IEEE Transactions on, vol. 41, pp. 77-88, 1994. 
[137] L. J. Trejo and M. J. Shensa, "Feature extraction of event-related potentials using wavelets: an application to human performance monitoring," Brain Lang., vol. 66, pp. 89107, 1999.

[138] T. Kalayci, O. Ozdamar and N. Erdol, "The use of wavelet transform as a preprocessor for the neural network detection of EEG spikes," in

Southeastcon'94. 'Creative Technology Transfer-A Global Affair'., Proceedings of the 1994 IEEE, 1994, pp. 1-3.

[139] D. M. Tucker, "Spatial sampling of head electrical fields: The geodesic sensor net," Electroencephalogr. Clin. Neurophysiol., vol. 87, pp. 154-163, 1993.

[140] S. J. Schiff, J. G. Milton, J. Heller and S. L. Weinstein, "Wavelet transforms and surrogate data for electroencephalographic spike and seizure localization (Journal Paper)," Optical Engineering, vol. 33, pp. 2162-2169, 1994.

[141] J. Raz, L. Dickerson and B. Turetsky, "A wavelet packet model of evoked potentials," Brain Lang., vol. 66, pp. 61-88, 1999.

[142] G. D. Fuller, Biofeedback: Methods and Procedures in Clinical Practice. Biofeedback Press, 1977.

[143] M. Sung, C. Marci and A. Pentland, "Journal of NeuroEngineering and Rehabilitation," Journal of Neuroengineering and Rehabilitation, vol. 2, pp. 0003-0002, 2005.

[144] T. M. Mitchell, "Machine learning. 1997," Burr Ridge, IL: McGraw Hill, 1997.

[145] E. Frank, Y. Wang, S. Inglis, G. Holmes and I. H. Witten, "Using model trees for classification," Mach. Learning, vol. 32, pp. 63-76, 1998.

[146] J. R. Quinlan, C4. 5: Programs for Machine Learning. Morgan kaufmann, 1993.

[147] J. R. Quinlan, "Bagging, boosting, and C4. 5," in Proceedings of the National Conference on Artificial Intelligence, 1996, pp. 725-730.

[148] J. R. Quinlan, "Induction of decision trees," Mach. Learning, vol. 1, pp. 81-106, 1986.

[149] C. A. Frantzidis, C. Bratsas, M. A. Klados, E. Konstantinidis, C. D. Lithari, A. B. Vivas, C. L. Papadelis, E. Kaldoudi, C. Pappas and P. D. Bamidis, "On the classification of emotional biosignals evoked while viewing affective pictures: an integrated data-mining- 
based approach for healthcare applications," Information Technology in Biomedicine, IEEE Transactions on, vol. 14, pp. 309-318, 2010.

[150] L. Breiman, "Random forests," Mach. Learning, vol. 45, pp. 5-32, 2001.

[151] A. Liaw and M. Wiener, "Classification and Regression by randomForest," $R$ News, vol. 2, pp. 18-22, 2002.

[152] J. Krajewski, S. Schnieder, D. Sommer, A. Batliner and B. Schuller, "Applying Multiple Classifiers and Non-Linear Dynamics Features for Detecting Sleepiness from Speech," Neurocomputing, 2011. 


\section{APPENDICES}

The following is the table of feature extracted from all the different ECG processing methods. The table displays a column showing which of these features were selected and not selected for the final 281 features used after feature space reduction step.

\begin{tabular}{|lll|}
\hline Feature & Number & Selected \\
\hline Featuresfrom DTCWT analysis & & \\
\hline std R_Det_I & 1 & not_selected \\
\hline enrgy R_Det_I1 & 2 & not_selected \\
\hline entropy R_Det_I1 & 3 & not_selected \\
\hline median R_Det_I1 & 4 & not_selected \\
\hline mean 1 R_Det_I1 & 5 & selected \\
\hline power R_Det_1 & 6 & not_selected \\
\hline min R_Det_I1 & 7 & not_selected \\
\hline max R_Det_I1 & 8 & not_selected \\
\hline slope R_Det_I1 & 9 & selected \\
\hline kurtosis R_Det_I1 & 10 & not_selected \\
\hline skewness R_Det_I1 & 11 & selected \\
\hline range R_Det_I1 & 12 & not_selected \\
\hline complexity R_Det_I1 & 13 & not_selected \\
\hline mobility R_Det_I1 & 14 & not_selected \\
\hline variance_pdfmean R_Det_I1 & 15 & not_selected \\
\hline log_varmean R_Det_I1 & 16 & not_selected \\
\hline mean_fftmean R_Det_I1 & 17 & not_selected \\
\hline sum_autoCorrelationmean R_Det_I1 & 18 & selected \\
\hline mean_autoCovariancemean R_Det_I1 & 19 & not_selected \\
\hline std R_Det_I2 & 20 & not_selected \\
\hline enrgy R_Det_I2 & 21 & not_selected \\
\hline entropy R_Det_I2 & 22 & not_selected \\
\hline median R_Det_12 & 23 & not_selected \\
\hline mean R_Det_I2 & 24 & selected \\
\hline power R_Det_12 & 25 & not_selected \\
\hline min R_Det_I2 & not_selected \\
\hline max R_Det_I2 & selected \\
\hline slope R_Det_12 & & \\
\hline
\end{tabular}




\begin{tabular}{|c|c|c|}
\hline kurtosis R_Det_I2 & 29 & not_selected \\
\hline skewness R_Det_I2 & 30 & not_selected \\
\hline range $\mathrm{R} \_$Det_I2 & 31 & selected \\
\hline complexity R_Det_I2 & 32 & not_selected \\
\hline mobility R_Det_I2 & 33 & selected \\
\hline variance_pdf R_Det_I2 & 34 & not_selected \\
\hline log_var R_Det_12 & 35 & not_selected \\
\hline mean_fft R_Det_I2 & 36 & not_selected \\
\hline sum_autoCorrelation R_Det_I2 & 37 & not_selected \\
\hline mean_autoCovariance R_Det_I2 & 38 & selected \\
\hline std R_Det_I3 & 39 & not_selected \\
\hline enrgy R_Det_I3 & 40 & not_selected \\
\hline entropy R_Det_I3 & 41 & not_selected \\
\hline median R_Det_I3 & 42 & not_selected \\
\hline mean R_Det_I3 & 43 & selected \\
\hline power R_Det_I3 & 44 & not_selected \\
\hline min R_Det_13 & 45 & not_selected \\
\hline max R_Det_I3 & 46 & not_selected \\
\hline slope R_Det_I3 & 47 & selected \\
\hline kurtosis R_Det_I3 & 48 & not_selected \\
\hline skewness R_Det_I3 & 49 & selected \\
\hline range R_Det_I3 & 50 & selected \\
\hline complexity R_Det_I3 & 51 & selected \\
\hline mobility R_Det_I3 & 52 & selected \\
\hline variance_pdf R_Det_I3 & 53 & selected \\
\hline log_var R_Det_I3 & 54 & not_selected \\
\hline mean_fft R_Det_I3 & 55 & not_selected \\
\hline sum_autoCorrelation R_Det_I3 & 56 & selected \\
\hline mean_autoCovariance R_Det_I3 & 57 & selected \\
\hline std R_Det_14 & 58 & not_selected \\
\hline enrgy R_Det_14 & 59 & not_selected \\
\hline entropy R_Det_14 & 60 & selected \\
\hline median R_Det_14 & 61 & not_selected \\
\hline mean R_Det_14 & 62 & selected \\
\hline power R_Det_I4 & 63 & not_selected \\
\hline min R_Det_14 & 64 & selected \\
\hline max R_Det_14 & 65 & not_selected \\
\hline
\end{tabular}




\begin{tabular}{|c|c|c|}
\hline slope R_Det_14 & 66 & selected \\
\hline kurtosis R_Det_I4 & 67 & not_selected \\
\hline skewness R_Det_14 & 68 & not_selected \\
\hline range R_Det_14 & 69 & selected \\
\hline complexity R_Det_14 & 70 & selected \\
\hline mobility R_Det_14 & 71 & selected \\
\hline variance_pdf R_Det_14 & 72 & not_selected \\
\hline log_var R_Det_14 & 73 & not_selected \\
\hline mean_fft R_Det_14 & 74 & not_selected \\
\hline sum_autoCorrelation R_Det_14 & 75 & selected \\
\hline mean_autoCovariance R_Det_14 & 76 & selected \\
\hline std R_Det_I5 & 77 & not_selected \\
\hline enrgy R_Det_I5 & 78 & not_selected \\
\hline entropy R_Det_I5 & 79 & selected \\
\hline median R_Det_I5 & 80 & not_selected \\
\hline mean R_Det_I5 & 81 & not_selected \\
\hline power R_Det_I5 & 82 & not_selected \\
\hline min R_Det_I5 & 83 & not_selected \\
\hline max R_Det_I5 & 84 & not_selected \\
\hline slope R_Det_I5 & 85 & selected \\
\hline kurtosis R_Det_I5 & 86 & not_selected \\
\hline skewness R_Det_I5 & 87 & selected \\
\hline range R_Det_I5 & 88 & selected \\
\hline complexity R_Det_I5 & 89 & selected \\
\hline mobility R_Det_I5 & 90 & not_selected \\
\hline variance_pdf R_Det_I5 & 91 & not_selected \\
\hline log_var R_Det_I5 & 92 & not_selected \\
\hline mean_fft R_Det_I5 & 93 & not_selected \\
\hline sum_autoCorrelation R_Det_I5 & 94 & selected \\
\hline mean_autoCovariance R_Det_I5 & 95 & selected \\
\hline std R_App_I5 & 96 & selected \\
\hline enrgy R_App_I5 & 97 & selected \\
\hline entropy R_App_I5 & 98 & selected \\
\hline median R_App_I5 & 99 & not_selected \\
\hline mean R_App_I5 & 100 & selected \\
\hline power R_App_I5 & 101 & selected \\
\hline min R_App_I5 & 102 & selected \\
\hline
\end{tabular}




\begin{tabular}{|c|c|c|}
\hline max R_App_I5 & 103 & selected \\
\hline slope R_App_I5 & 104 & selected \\
\hline kurtosis R_App_I5 & 105 & selected \\
\hline skewness R_App_I5 & 106 & not_selected \\
\hline range $\mathrm{R} \_$App_I5 & 107 & selected \\
\hline complexity R_App_I5 & 108 & selected \\
\hline mobility R_App_I5 & 109 & not_selected \\
\hline variance_pdf R_App_I5 & 110 & selected \\
\hline log_var R_App_I5 & 111 & selected \\
\hline mean_fft R_App_I5 & 112 & selected \\
\hline sum_autoCorrelation R_App_I5 & 113 & selected \\
\hline mean_autoCovariance R_App_I5 & 114 & selected \\
\hline std I_Det_I1 & 115 & selected \\
\hline enrgy I_Det_I1 & 116 & not_selected \\
\hline entropy I_Det_I1 & 117 & not_selected \\
\hline median I_Det_I1 & 118 & not_selected \\
\hline I_Det_|1 I_Det_I1 & 119 & selected \\
\hline power I_Det_I1 & 120 & not_selected \\
\hline min I_Det_I1 & 121 & not_selected \\
\hline max I_Det_I1 & 122 & not_selected \\
\hline slope I_Det_I1 & 123 & selected \\
\hline kurtosis I_Det_I1 & 124 & not_selected \\
\hline skewness I_Det_I1 & 125 & selected \\
\hline range I_Det_I1 & 126 & selected \\
\hline complexity I_Det_I1 & 127 & not_selected \\
\hline mobility I_Det_I1 & 128 & not_selected \\
\hline variance_pdf I_Det_I1 & 129 & not_selected \\
\hline log_var I_Det_I1 & 130 & not_selected \\
\hline mean_fft I_Det_I1 & 131 & not_selected \\
\hline $\begin{array}{l}\text { sum_autoCorrelation } \\
\text { I_Det_I1 }\end{array}$ & 132 & selected \\
\hline mean_autoCovariance I_Det_I1 & 133 & selected \\
\hline std I_Det_I2 & 134 & not_selected \\
\hline enrgy I_Det_I2 & 135 & not_selected \\
\hline entropy I_Det_12 & 136 & not_selected \\
\hline median I_Det_I2 & 137 & not_selected \\
\hline I_Det_I1 I_Det_I2 & 138 & selected \\
\hline
\end{tabular}




\begin{tabular}{|c|c|c|}
\hline power I_Det_I2 & 139 & not_selected \\
\hline min I_Det_I2 & 140 & not_selected \\
\hline max I_Det_I2 & 141 & not_selected \\
\hline slope I_Det_I2 & 142 & selected \\
\hline kurtosis I_Det_12 & 143 & not_selected \\
\hline skewness I_Det_12 & 144 & selected \\
\hline range I_Det_I2 & 145 & selected \\
\hline complexity I_Det_I2 & 146 & selected \\
\hline mobility I_Det_I2 & 147 & selected \\
\hline variance_pdf I_Det_I2 & 148 & not_selected \\
\hline log_var I_Det_I2 & 149 & not_selected \\
\hline mean_fft I_Det_I2 & 150 & not_selected \\
\hline $\begin{array}{l}\text { sum_autoCorrelation } \\
\text { I_Det_12 }\end{array}$ & 151 & selected \\
\hline mean_autoCovariance I_Det_I2 & 152 & selected \\
\hline std I_Det_I3 & 153 & not_selected \\
\hline enrgy I_Det_I3 & 154 & not_selected \\
\hline entropy I_Det_I3 & 155 & selected \\
\hline median I_Det_I3 & 156 & not_selected \\
\hline I_Det_I1 I_Det_|3 & 157 & selected \\
\hline power I_Det_I3 & 158 & not_selected \\
\hline min I_Det_I3 & 159 & not_selected \\
\hline max I_Det_13 & 160 & not_selected \\
\hline slope I_Det_I3 & 161 & selected \\
\hline kurtosis I_Det_13 & 162 & not_selected \\
\hline skewness I_Det_I3 & 163 & selected \\
\hline range I_Det_I3 & 164 & selected \\
\hline complexity I_Det_I3 & 165 & selected \\
\hline mobility I_Det_13 & 166 & selected \\
\hline variance_pdf I_Det_I3 & 167 & selected \\
\hline log_var I_Det_I3 & 168 & not_selected \\
\hline mean_fft I_Det_I3 & 169 & not_selected \\
\hline $\begin{array}{l}\text { sum_autoCorrelation } \\
\text { I_Det_I3 }\end{array}$ & 170 & selected \\
\hline mean_autoCovariance I_Det_I3 & 171 & selected \\
\hline std I_Det_I4 & 172 & not_selected \\
\hline enrgy I_Det_I4 & 173 & selected \\
\hline
\end{tabular}




\begin{tabular}{|c|c|c|}
\hline entropy I_Det_14 & 174 & selected \\
\hline median I_Det_14 & 175 & selected \\
\hline I_Det_I1 I_Det_I4 & 176 & selected \\
\hline power I_Det_14 & 177 & selected \\
\hline min I_Det_14 & 178 & selected \\
\hline max I_Det_14 & 179 & selected \\
\hline slope I_Det_14 & 180 & selected \\
\hline kurtosis I_Det_14 & 181 & selected \\
\hline skewness I_Det_14 & 182 & selected \\
\hline range I_Det_|4 & 183 & selected \\
\hline complexity I_Det_14 & 184 & selected \\
\hline mobility I_Det_14 & 185 & selected \\
\hline variance_pdf I_Det_14 & 186 & selected \\
\hline log_var I_Det_14 & 187 & selected \\
\hline mean_fft I_Det_14 & 188 & selected \\
\hline $\begin{array}{l}\text { sum_autoCorrelation } \\
\text { I_Det_14 }\end{array}$ & 189 & selected \\
\hline mean_autoCovariance I_Det_I4 & 190 & selected \\
\hline std I_Det_I5 & 191 & selected \\
\hline enrgy I_Det_I5 & 192 & selected \\
\hline entropy I_Det_I5 & 193 & selected \\
\hline median I_Det_I5 & 194 & selected \\
\hline I_Det_I1 I_Det_I5 & 195 & selected \\
\hline power I_Det_I5 & 196 & selected \\
\hline min I_Det_I5 & 197 & selected \\
\hline max I_Det_I5 & 198 & selected \\
\hline slope I_Det_I5 & 199 & selected \\
\hline kurtosis I_Det_I5 & 200 & selected \\
\hline skewness I_Det_I5 & 201 & selected \\
\hline range I_Det_I5 & 202 & selected \\
\hline complexity I_Det_I5 & 203 & selected \\
\hline mobility I_Det_I5 & 204 & selected \\
\hline variance_pdf I_Det_I5 & 205 & selected \\
\hline log_var I_Det_I5 & 206 & selected \\
\hline mean_fft I_Det_I5 & 207 & selected \\
\hline $\begin{array}{l}\text { sum_autoCorrelation } \\
\text { I_Det_I5 }\end{array}$ & 208 & selected \\
\hline
\end{tabular}




\begin{tabular}{|c|c|c|}
\hline mean_autoCovariance I_Det_I5 & 209 & selected \\
\hline Entropy of Levels_Final_Feature & 210 & selected \\
\hline \multicolumn{3}{|l|}{ Features from ST analysis } \\
\hline mean(mean_of_freq) & 211 & selected \\
\hline sum(mean_of_freq) & 212 & selected \\
\hline mean_Xcovariance(mean_of_freq) & 213 & selected \\
\hline sum_Xcorrelation(mean_of_freq) & 214 & selected \\
\hline Log_var(mean_of_freq) & 215 & selected \\
\hline mean(sum_of_freq) & 216 & selected \\
\hline sum(sum_of_freq) & 217 & selected \\
\hline mean_Xcovariance(sum_of_freq) & 218 & selected \\
\hline sum_Xcorrelation(sum_of_freq) & 219 & selected \\
\hline Log_var(sum_of_freq) & 220 & selected \\
\hline mean(product_of_freq) & 221 & selected \\
\hline sum(product_of_freq) & 222 & not_selected \\
\hline mean_Xcovariance(product_of_freq) & 223 & not_selected \\
\hline sum_Xcorrelation(product_of_freq) & 224 & selected \\
\hline Log_var(product_of_freq) & 225 & selected \\
\hline mean(std_of_freq) & 226 & not_selected \\
\hline sum(std_of_freq) & 227 & selected \\
\hline mean_Xcovariance(std_of_freq) & 228 & selected \\
\hline sum_Xcorrelation(std_of_freq) & 229 & selected \\
\hline Log_var(std_of_freq) & 230 & selected \\
\hline mean(range_of_freq) & 231 & selected \\
\hline $\operatorname{sum}(e$ & 232 & selected \\
\hline mean_Xcovariance(range_of_freq) & 233 & selected \\
\hline sum_Xcorrelation(range_of_freq) & 234 & selected \\
\hline Log_var(range_of_freq) & 235 & selected \\
\hline Mean_Max_Frequencies & 236 & selected \\
\hline Mean_Absolute_Deviation_Freq & 237 & not_selected \\
\hline \multicolumn{3}{|l|}{ Features from QRS Complex Analysis } \\
\hline $\min (R-R)$ & 238 & selected \\
\hline $\max (\mathrm{R}-\mathrm{R})$ & 239 & selected \\
\hline std (R-R) & 240 & selected \\
\hline energy (R-R) & 241 & selected \\
\hline power (R-R) & 242 & selected \\
\hline mean $(\mathrm{R}-\mathrm{R})$ & 243 & selected \\
\hline
\end{tabular}




\begin{tabular}{|c|c|c|}
\hline median (R-R) & 244 & selected \\
\hline slope $(R-R)$ & 245 & selected \\
\hline kurtosis (R-R) & 246 & selected \\
\hline range (R-R) & 247 & selected \\
\hline complexity (R-R) & 248 & selected \\
\hline mobility (R-R) & 249 & selected \\
\hline log_var (R-R) & 250 & selected \\
\hline mean_fft (R-R) & 251 & selected \\
\hline var_pdf (R-R) & 252 & selected \\
\hline sum_Xcorrelation (R-R) & 253 & selected \\
\hline mean_Xcovariance (R-R) & 254 & selected \\
\hline $\min (Q-Q)$ & 255 & selected \\
\hline $\max (Q-Q)$ & 256 & selected \\
\hline $\operatorname{std}(Q-Q)$ & 257 & selected \\
\hline energy (Q-Q) & 258 & selected \\
\hline power (Q-Q) & 259 & selected \\
\hline mean (Q-Q) & 260 & selected \\
\hline median (Q-Q) & 261 & selected \\
\hline slope (Q-Q) & 262 & selected \\
\hline kurtosis (Q-Q) & 263 & selected \\
\hline range (Q-Q) & 264 & selected \\
\hline complexity (Q-Q) & 265 & selected \\
\hline mobility (Q-Q) & 266 & selected \\
\hline log_var (Q-Q) & 267 & selected \\
\hline mean_fft (Q-Q) & 268 & selected \\
\hline var_pdf (Q-Q) & 269 & selected \\
\hline sum_Xcorrelation (Q-Q) & 270 & selected \\
\hline mean_Xcovariance (Q-Q) & 271 & not_selected \\
\hline $\min (S-S)$ & 272 & selected \\
\hline $\max (\mathrm{S}-\mathrm{S})$ & 273 & selected \\
\hline std (S-S) & 274 & selected \\
\hline energy (S-S) & 275 & selected \\
\hline power (S-S) & 276 & selected \\
\hline mean (S-S) & 277 & selected \\
\hline median (S-S) & 278 & selected \\
\hline slope (S-S) & 279 & selected \\
\hline kurtosis (S-S) & 280 & selected \\
\hline
\end{tabular}




\begin{tabular}{|c|c|c|}
\hline range (S-S) & 281 & selected \\
\hline complexity (S-S) & 282 & selected \\
\hline mobility (S-S) & 283 & selected \\
\hline log_var (S-S) & 284 & selected \\
\hline mean_fft (S-S) & 285 & selected \\
\hline var_pdf (S-S) & 286 & selected \\
\hline sum_Xcorrelation (S-S) & 287 & selected \\
\hline mean_Xcovariance (S-S) & 288 & not_selected \\
\hline $\min (\mathrm{Q}-\mathrm{R})$ & 289 & selected \\
\hline $\max (\mathrm{Q}-\mathrm{R})$ & 290 & selected \\
\hline std (Q-R) & 291 & selected \\
\hline energy (Q-R) & 292 & selected \\
\hline power (Q-R) & 293 & selected \\
\hline mean $(Q-R)$ & 294 & selected \\
\hline median (Q-R) & 295 & selected \\
\hline slope (Q-R) & 296 & selected \\
\hline kurtosis (Q-R) & 297 & selected \\
\hline range $(Q-R)$ & 298 & selected \\
\hline complexity (Q-R) & 299 & selected \\
\hline mobility (Q-R) & 300 & selected \\
\hline log_var (Q-R) & 301 & selected \\
\hline mean_fft (Q-R) & 302 & selected \\
\hline var_pdf (Q-R) & 303 & selected \\
\hline sum_Xcorrelation (Q-R) & 304 & selected \\
\hline mean_Xcovariance (Q-R) & 305 & selected \\
\hline $\min (\mathrm{R}-\mathrm{S})$ & 306 & selected \\
\hline $\max (\mathrm{R}-\mathrm{S})$ & 307 & selected \\
\hline std (R-S) & 308 & selected \\
\hline energy (R-S) & 309 & selected \\
\hline power (R-S) & 310 & selected \\
\hline mean (R-S) & 311 & selected \\
\hline median (R-S) & 312 & selected \\
\hline slope (R-S) & 313 & selected \\
\hline kurtosis (R-S) & 314 & selected \\
\hline range (R-S) & 315 & selected \\
\hline complexity (R-S) & 316 & selected \\
\hline mobility (R-S) & 317 & selected \\
\hline
\end{tabular}




\begin{tabular}{|c|c|c|}
\hline log_var (R-S) & 318 & selected \\
\hline mean_fft (R-S) & 319 & selected \\
\hline var_pdf (R-S) & 320 & selected \\
\hline sum_Xcorrelation (R-S) & 321 & selected \\
\hline mean_Xcovariance (R-S) & 322 & selected \\
\hline $\min (\mathrm{Q}-\mathrm{S})$ & 323 & not_selected \\
\hline $\max (\mathrm{Q}-\mathrm{S})$ & 324 & selected \\
\hline std (Q-S) & 325 & selected \\
\hline energy (Q-S) & 326 & selected \\
\hline power (Q-S) & 327 & selected \\
\hline mean $(Q-S)$ & 328 & selected \\
\hline median (Q-S) & 329 & selected \\
\hline slope (Q-S) & 330 & selected \\
\hline kurtosis (Q-S) & 331 & selected \\
\hline range $(Q-S)$ & 332 & selected \\
\hline complexity (Q-S) & 333 & selected \\
\hline mobility (Q-S) & 334 & not_selected \\
\hline log_var (Q-S) & 335 & selected \\
\hline mean_fft (Q-S) & 336 & selected \\
\hline var_pdf (Q-S) & 337 & selected \\
\hline sum_Xcorrelation (Q-S) & 338 & selected \\
\hline mean_Xcovariance (Q-S) & 339 & selected \\
\hline $\min (R / S)$ & 340 & selected \\
\hline $\max (\mathrm{R} / \mathrm{S})$ & 341 & not_selected \\
\hline std $(R / S)$ & 342 & selected \\
\hline energy $(R / S)$ & 343 & selected \\
\hline power (R/S) & 344 & selected \\
\hline mean $(\mathrm{R} / \mathrm{S})$ & 345 & not_selected \\
\hline median (R/S) & 346 & not_selected \\
\hline slope (R/S) & 347 & not_selected \\
\hline kurtosis (R/S) & 348 & selected \\
\hline range $(\mathrm{R} / \mathrm{S})$ & 349 & selected \\
\hline complexity (R/S) & 350 & selected \\
\hline mobility (R/S) & 351 & selected \\
\hline log_var (R/S) & 352 & selected \\
\hline mean_fft (R/S) & 353 & selected \\
\hline var_pdf (R/S) & 354 & selected \\
\hline
\end{tabular}




\begin{tabular}{|c|c|c|}
\hline sum_Xcorrelation (R/S) & 355 & not_selected \\
\hline mean_Xcovariance $(\mathrm{R} / \mathrm{S})$ & 356 & selected \\
\hline $\min (\mathrm{Q} / \mathrm{R})$ & 357 & selected \\
\hline $\max (\mathrm{Q} / \mathrm{R})$ & 358 & not_selected \\
\hline std $(Q / R)$ & 359 & not_selected \\
\hline energy $(\mathrm{Q} / \mathrm{R})$ & 360 & selected \\
\hline power (Q/R) & 361 & not_selected \\
\hline mean $(\mathrm{Q} / \mathrm{R})$ & 362 & selected \\
\hline median $(\mathrm{Q} / \mathrm{R})$ & 363 & selected \\
\hline slope (Q/R) & 364 & selected \\
\hline kurtosis (Q/R) & 365 & selected \\
\hline range $(\mathrm{Q} / \mathrm{R})$ & 366 & selected \\
\hline complexity (Q/R) & 367 & selected \\
\hline mobility $(\mathrm{Q} / \mathrm{R})$ & 368 & selected \\
\hline log_var (Q/R) & 369 & selected \\
\hline mean_fft (Q/R) & 370 & selected \\
\hline var_pdf $(Q / R)$ & 371 & not_selected \\
\hline sum_Xcorrelation $(\mathrm{Q} / \mathrm{R})$ & 372 & selected \\
\hline mean_Xcovariance $(\mathrm{Q} / \mathrm{R})$ & 373 & not_selected \\
\hline $\min (\mathrm{O} / \mathrm{S})$ & 374 & selected \\
\hline $\max (\mathrm{Q} / \mathrm{S})$ & 375 & selected \\
\hline $\operatorname{std}(\mathrm{Q} / \mathrm{S})$ & 376 & selected \\
\hline energy $(\mathrm{Q} / \mathrm{S})$ & 377 & selected \\
\hline power $(\mathrm{Q} / \mathrm{S})$ & 378 & selected \\
\hline mean $(\mathrm{Q} / \mathrm{S})$ & 379 & selected \\
\hline median (Q/S) & 380 & selected \\
\hline slope (Q/S) & 381 & selected \\
\hline kurtosis (Q/S) & 382 & selected \\
\hline range $(\mathrm{Q} / \mathrm{S})$ & 383 & selected \\
\hline complexity (Q/S) & 384 & selected \\
\hline mobility (Q/S) & 385 & selected \\
\hline log_var (Q/S) & 386 & selected \\
\hline mean_fft $(\mathrm{Q} / \mathrm{S})$ & 387 & selected \\
\hline var_pdf (Q/S) & 388 & selected \\
\hline sum_Xcorrelation $(\mathrm{Q} / \mathrm{S})$ & 389 & not_selected \\
\hline mean_Xcovariance $(\mathrm{Q} / \mathrm{S})$ & 390 & selected \\
\hline $\mathrm{LF} / \mathrm{HF}$ & 391 & selected \\
\hline
\end{tabular}




\begin{tabular}{|lll|}
\hline LF & 392 & selected \\
\hline HF & 393 & selected \\
\hline Mean_HRV & 394 & selected \\
\hline Std_HRV & 395 & selected \\
\hline RMSSD & 396 & selected \\
\hline
\end{tabular}




\title{
CURRICULUM VITAE
}

\begin{abstract}
ASHWIN BELLE
Specializations: Signal Processing, Image Processing, Biomedical R\&D, Artificial Intelligence, Machine Learning, Embedded Systems, Technical Email: bellea@vcu.edu DATE OF BIRTH FEBRUARY $11^{\mathrm{TH}} 1986$ PLACE OF BIRTH KARNATAKA, INIDA COUNTRY OF CURRENT CITIZENSHIP: INDIA
\end{abstract}

Writing.

\section{Education}

Master of Science in Computer Science Specialization in Embedded Systems

Dec 2008 Virginia Commonwealth University, School of Engineering, VA, USA

Bachelor Computer Science and Engineering Jun 2007 Dayananda Sagar College of Engineering, VT University, Bangalore, India

\section{Publications}

Conference Publications

- Ashwin Belle, Michael Pfaffenberger, Rosalyn H. Hargraves, Kayvan Najarian. “An Automated Decision Making System for Detecting Loss of Attention in Individuals Using Real Time Processing of Electroencephalogram”, Biosignal Interpretation- 7th International Workshop 2012.

- Pavani Davuluri, Ashwin Belle, Jie Wu, Y Tang, C Cockrell, K Ward, R Hargraves, Kayvan Najarian, "An Image Processing and Machine Learning Based Computer-Aided Decision Support System for Traumatic Pelvic Injury’, Biosignal Interpretation- 7th International Workshop 2012.

- Ashwin Belle, Rosalyn Hobson, Kayvan Najarian, "A physiologica Signal Processing system for optimal Engagement and Attention Detection.”, International Workshop on Biomedical and Health Informatics, IEEE BIBM, Nov 2011.

- Ashwin Belle, S.Y. Ji, S. Ansari, R. Hakimzadeh, K. Ward, K. Najarian, "Frustration Detection with Electrocardiogram Signal using Wavelet Transform", The Second International Conference on Advances in Biotechnologies (BIOTECHNO 2010), Cancun, Mexico, March, 2010.

- Sardar Ansari, Ashwin Belle, Rosalyn Hobson, Kevin Ward, Kayvan Najarian, "Reduction of Periodic Motion Artifacts from Impedance Plethysmography.”, International Workshop on Biomedical and Health Informatics, BIBM, Nov 2011.

- Pavani Davuluri, Jie Wu, Ashwin Belle, Charles Cockrell, Yang Tang, Kevin Ward, Kayvan Najarian and Rosalyn Hargraves, "A Hybrid Approach for Hemorrhage Segmentation in Pelvic CT Scans", International Workshop on Biomedical and Health Informatics, BIBM, Nov 2011.

- Jie Wu, Pavani Davuluri, Ashwin Belle, Charles Cockrell, Yang Tang, Kevin Ward, Kayvan Najarian and Rosalyn Hargraves "Fracture Detection and Quantitative 
Measurement of Displacement in Pelvic CT images.”, International Workshop on Biomedical and Health Informatics, BIBM, Nov 2011.

- S. Ansari, Ashwin Belle, K. Ward, and K. Najarian, "Impedance Plethysmography on the arms: Respiration Monitoring", The First Workshop on Knowledge Engineering, Discovery and Dissemination in Health, part of The 2010 IEEE International Conference on Bioinformatics \& Biomedicine (BIBM2010), Hong Kong, Dec 2010.

- Ashwin Belle, Dr Pidaparti, "Portable Integrated Wireless Sensor System for Damage Mnitoring”, AIAA conference April 2008 University of Maryland, 2008.

Book Chapter Publications

- Ashwin Belle, Soo-Yeon Ji, Wenan Chen, Toan Huynh, and Kayvan Najarian, Chapter: "Rule-Based Computer Aided Decision Making for Traumatic Brain Injuries", Machine Learning in Healthcare Informatics, to be published by Springer, In Press, 2012.

Journal Publication

- Ashwin Belle, Rosalyn H Hobson, Kayvan Najarian, 'An Automated Optimal Engagement and Attention Detection System Using Electrocardiogram’, Journal of Computational and Mathematical Methods in Medicine, July 2012.

- Wenan Chen, Ashwin Belle, Charles Cockrell, Kevin R. Ward, Kayvan Najarian, 'Automated Midline Shift and Intracranial Pressure Estimation based on Brain CT Images', Journal of Visual Experiment, In Press, 2012.

- Ramana M. Pidaparti, Ashwin Belle, and Ju Wang; "Development of a Portable Integrated Wireless Sensor Module for Structural Damage Monitoring”, Tech Science press, SDHM journal Vol.5, No.4, 2009.

\title{
Experience
}

\author{
Research Assistant Virginia Commonwealth University \\ Aug 2007-present \\ Physiological signal based attention detection system \\ PHD Dissertation, School of Engineering VCU. \\ Computer-Aided Decision Support System for Traumatic Pelvic Injuries \\ School of Engineering (Computer Science and Electronics Department- Collaborative Research) \\ Intra-oral image analysis and admonition system \\ VCU-MCV, School of Dentistry and School of Engineering \\ Portable Wireless Sensor System for Damage Monitoring \\ Master's thesis, Computer Science/Mechanical department, VCU
}

\section{Research Associat Veterans Affair Hospital, McGuire Research Institute, VA Oct 2010-Jan 2012}

Real-Time Video Monitoring System for Hemodialysis Pilot Research project, targeted for NIH and VA funding.

\section{Awards and ScholArships}

Who's Who Among Students in American Universities and Colleges Award 2011-2012

Outstanding Graduate Researcher Award 2011-2012

Won Third place for 'Top Publications and Presentations - Graduate Level' March 2008

Research Assistantship, PhD and Masters 2007-2012 